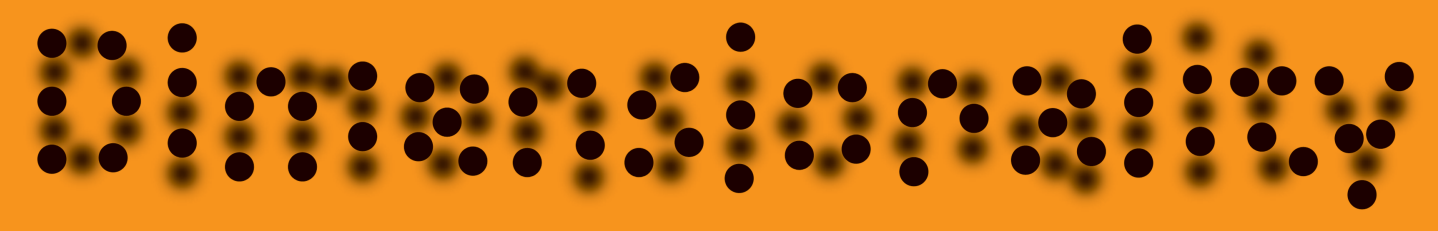

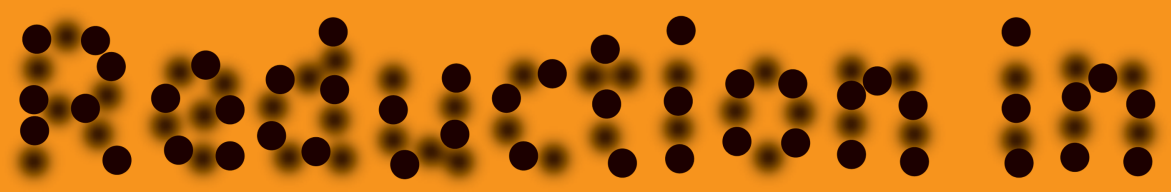

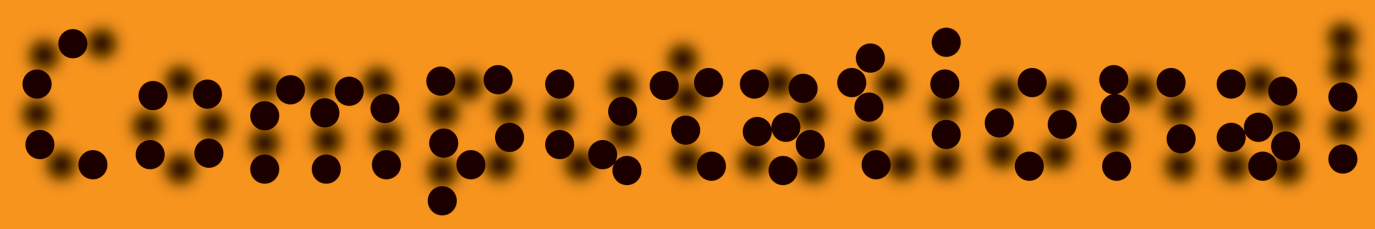

ใُ

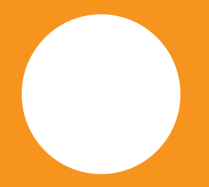

O.V. (Alyona) Ivanova

ISBN 978-90-365-2997-6

March, 2010 



\section{DIMENSIONALITY REDUCTION IN COMPUTATIONAL PHOTONICS}


The research presented in this thesis was carried out at the group of Applied Analysis and Mathematical Physics (AAMP), the faculty of Electrical Engineering, Mathematics and Computer Science, and MESA+ Institute for Nanotechnology, University of Twente, PO Box 217, 7500 AE Enschede, The Netherlands.

\section{MESA+ UNIVERSITY OF TWENTE.}

The work was supported by the national nanotechnology program NanoNed, project TOE.7143.

\section{nano ned}

Cover design by Serhiy Chebotaryov (http://che.co.ua).

Printed by Wöhrmann Print Service, Zutphen, The Netherlands.

Copyright (c) O.V. (Alyona) Ivanova, Enschede, The Netherlands, 2010.

DOI 10.3990/1.9789036529976

ISBN 978-90-365-2997-6 


\title{
DIMENSIONALITY REDUCTION IN COMPUTATIONAL PHOTONICS
}

\author{
DISSERTATION
}

to obtain

the degree of doctor at the University of Twente, on the authority of the rector magnificus, prof. dr. H. Brinksma,

on account of the decision of the graduation committee, to be publicly defended

on Thursday 25 March 2010 at 16:45

by

Olena Ivanova

born on 27 March 1983

in Kharkiv, Ukraine 
Dit proefschrift is goedgekeurd door de promotor, prof. dr. E.W.C. van Groesen

en de assistent-promotor, dr. M. Hammer 
Samenstelling van de promotiecommissie:

Voorzitter en sectretaris:

prof. dr. ir. A.J. Mouthaan

Universiteit Twente

Promotor:

prof. dr. ir. E.W.C. van Groesen Universiteit Twente

Assistent-promotor:

dr. M. Hammer

Universiteit Twente

Leden:

prof. dr. ir. G.J.M. Krijnen

Universiteit Twente

dr. H.J.W.M. Hoekstra

Universiteit Twente

prof. dr. A.I. Nosich

National Academy of Sciences of Ukraine

dr. J.J.G.M. van der Tol

Technische Universiteit Eindhoven

prof. dr. H.P. Urbach

Technische Universiteit Delft 



\section{Contents}

\section{Introduction}

2 Scalar mode problems $\quad 25$

2.1 Introduction . . . . . . . . . . . . . . . . 25

2.2 Variational form of the mode problem . . . . . . . . . 27

2.3 Variational mode expansion method . . . . . . . . . 28

2.3.1 Modal basis functions . . . . . . . . . . . . . 32

2.3.2 Relation with the Effective Index Method . . . . . . 33 
2.4 Numerical results and comparisons . . . . . . . . . . 34

2.4.1 Rib waveguide . . . . . . . . . . . . . . . 34

2.4.2 Four evanescently coupled ribs . . . . . . . . . . . . . 38

2.4.3 3D coupler . . . . . . . . . . . . . . . 39

2.5 Concluding remarks . . . . . . . . . . . . . . . . 41

2.6 Appendix . . . . . . . . . . . . . . . . . . . . . . 42

3 Vectorial mode problems $\quad 45$

3.1 Introduction . . . . . . . . . . . . . . . 45

3.2 Variational form of the vectorial mode problem . . . . . 47

3.3 Slab modes . . . . . . . . . . . . . . . . 48

3.4 Modal field ansatz . . . . . . . . . . . . . . 50

3.5 Reduced problem . . . . . . . . . . . . . 52

3.6 Method of solution . . . . . . . . . . . . . 53

3.6.1 Arbitrary refractive index distribution: Finite Element Method . . . . . . . . . . . . . . 54

3.6.2 Piecewise constant refractive index distribution . . . 56

3.7 Relation with the Effective Index Method . . . . . . . 57

3.7.1 TE polarization . . . . . . . . . . . . 5 58

3.7.2 TM polarization . . . . . . . . . . . . 59

3.8 Relation with the Film Mode Matching Method . . . . . . . 61

3.9 Numerical results . . . . . . . . . . . . . . . . . . . 63

3.9.1 Box-shaped waveguide . . . . . . . . . . . 63

3.9 .2 Rectangular rib waveguide . . . . . . . . . . 66

3.9.3 Waveguide with non-rectangular piecewise constant cross-section . . . . . . . . . . . . . . . . . . . 68

3.9 .4 Indiffused waveguide . . . . . . . . . . . . 70

3.10 Concluding remarks . . . . . . . . . . . . . . . . 71

$4 \quad 2$ D scattering problems $\quad 73$

4.1 Introduction . . . . . . . . . . . . . 73

4.2 2D scattering problems . . . . . . . . . . . . 75

4.2.1 Dimensionality reduction . . . . . . . . . . . 76

4.2.2 Basis modes $X_{j}$ defined using Perfectly Matched Layers 77

4.2.3 Method of solution of the reduced problem . . . . . . 79

4.2.4 Relation with the Effective Index Method . . . . . 8 83

4.3 Numerical results . . . . . . . . . . . . . . . . . . . 83

4.3.1 Waveguide Bragg grating . . . . . . . . . . . . . . 84 
4.3.2 Waveguide Bragg grating perturbed by a nanosized probe ..................... 85

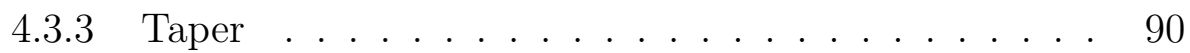

4.4 Concluding remarks . . . . . . . . . . . . . . . . . . . 91

$5 \quad 3 D$ scattering problems $\quad 93$

5.1 Introduction . . . . . . . . . . . . . . . . 93

5.2 Variational form of 3D scattering problems in optics . . . 9

5.3 General approximation . . . . . . . . . . . . . 96

5.4 Reduced problem . . . . . . . . . . . . . . . . . . . 98

5.5 Weak formulation . . . . . . . . . . . . . . 100

5.6 Transparent Influx Boundary Conditions . . . . . . . . . 100

5.6.1 Remarks . . . . . . . . . . . . . . 103

5.7 Choice of the expansion basis . . . . . . . . . . . 104

5.8 Relation with the Effective Index Method . . . . . . . . 104

5.8.1 TE polarization . . . . . . . . . . . 105

5.8 .2 TM polarization . . . . . . . . . . . . 106

5.9 Numerical results . . . . . . . . . . . . . . . . . . . . 109

5.9 .1 Silicon on insulator waveguide . . . . . . . . . 109

5.9.2 Photonic crystal slab waveguide . . . . . . . . . . 114

5.10 Conclusions . . . . . . . . . . . . . . . . 120

6 Conclusions and recommendations $\quad 121$

$\begin{array}{ll}\text { Bibliography } & 125\end{array}$

\begin{tabular}{l|l} 
Summary & 137
\end{tabular}

$\begin{array}{ll}\text { Samenvatting } & 141\end{array}$

$\begin{array}{ll}\text { Acknowledgments } & 145\end{array}$

$\begin{array}{ll}\text { About the author } & 147\end{array}$ 



\section{Introduction}

\section{$1.1 \quad$ Introduction}

These days, society is becoming more and more reliant on high-speed data communication. Broadband internet, high definition digital television and telephone services are all part of nearly everybody's daily life - and our use of these resources is ever increasing. New applications are popping up on a daily basis, with internet users' hunger for video-on-demand (like YouTube), interactive television, and file sharing services creating an enormous demand for data bandwidth. In many places, it is now already possible to have an optical fiber connection directly into your living room, offering a bandwidth of 100 megabit per second or more.

All this data traffic needs to be transported to the correct location. The majority of this transport is done on optical fibers [20], [83]. These fibers are made of special glasses, and are able to guide light - even around bends, see Figure 1.1 - for great distances; typical optical fibers (e.g. Corning SMF28e [19]) lose less than $5 \%$ power over a distance of one kilometer. The light travelling through such fibers can be modulated - switched 'on' and 'off' at very high rates, and thus digital information can be sent down the fiber to a receiver.

As a matter of fact, an optical fiber has an enormous capacity for data transport. If all available capacity could be used, a single optical fiber would be more than sufficient to handle all traffic that flows through AMS-IX [1], the main Dutch Internet Exchange. However, due to limitations of the electronic chips and the devices that modulate the light, it is not possible to directly use all that capacity. For this reason, a trick is employed: Multiple 


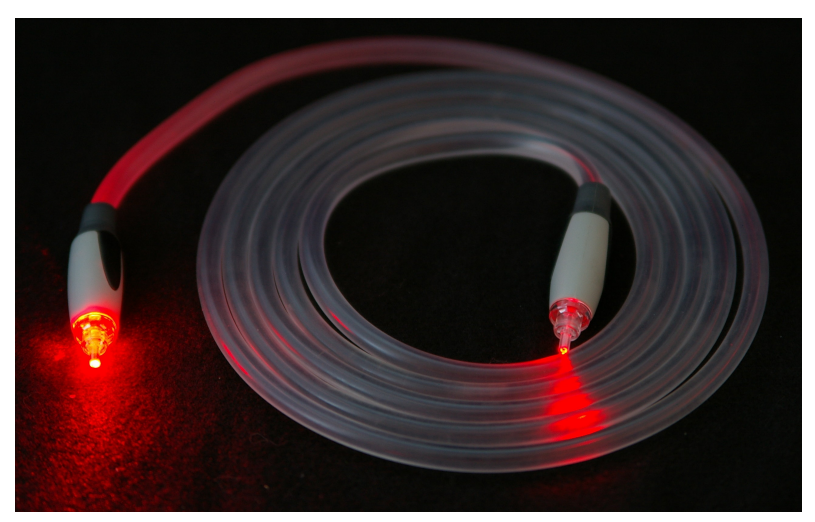

Figure 1.1: Optical fiber, illuminated by a red laser. Image source www.wikipedia.org, user Hustvedt.

colours of light are modulated independently and sent down the fiber. You can compare this with those silly $3 \mathrm{D}$ glasses with red and green lenses: one eye only sees the red image on the screen, and the other sees only the green one; their combination tricks our mind into seeing a 3D image. The red image on the screen is thus one 'channel' of information, while the green one is another channel. In exactly the same way, different colours can be transmitted through a fiber, where each colour carries its own information stream.

In a network of optical fibers, the data has to be directed to the correct end user; you do not want your neighbour to receive information that was meant for you. It may be desirable to send one colour of light along one channel, and another colour along the other channel. To do this steering of light, optical chips may be employed. You can compare optical chips to electronic microchips - except the former process light (photons) instead of electrons.

Another application area of optical chips is sensing. The speed of light may be affected by certain antibodies or chemicals in a material, e.g. when a receptor layer selectively captures certain bio-molecules. Since in optical chips, the interaction of the light with these materials can be measured very precisely and in a minute volume, only tiny amounts of blood or other fluids or gases are needed to perform an analysis. This allows for faster, more accurate, cheaper and more portable medical diagnostic equipment. Such equipment is especially important in developing countries; cheap and 
portable equipment can be deployed at many more hospitals and clinics than what is currently possible.

\subsection{Optical chips}

Light impinging on the boundary between two materials is partially transmitted and partially reflected, depending, among others, on the refractive index (which relates to the speed of light in that material) of the two materials. In certain cases, the light is even completely reflected - which means that if two of these interfaces are placed parallel to each other, light can be trapped in-between. This phenomenon of total internal reflection can also be used to trap and guide light in three dimensions: so-called waveguides with proper dimensions guide light without intrinsic losses. If designed correctly, these optical waveguides can even steer light around tiny bends with a radius of curvature smaller than the thickness of a human hair [87].

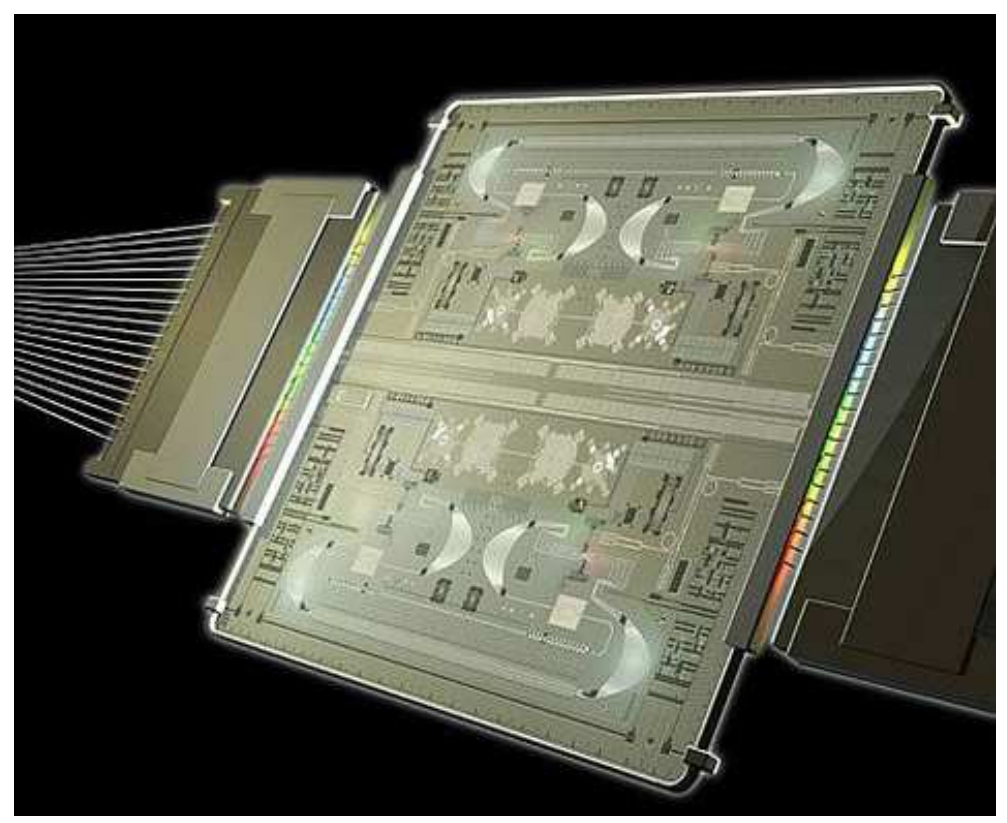

Figure 1.2: Artist's impression of a complex optical chip. Image source www. infinera. com.

Chips containing structures that may guide and manipulate light are called (integrated) optical chips; for examples see Figures 1.2 and 1.3. In 

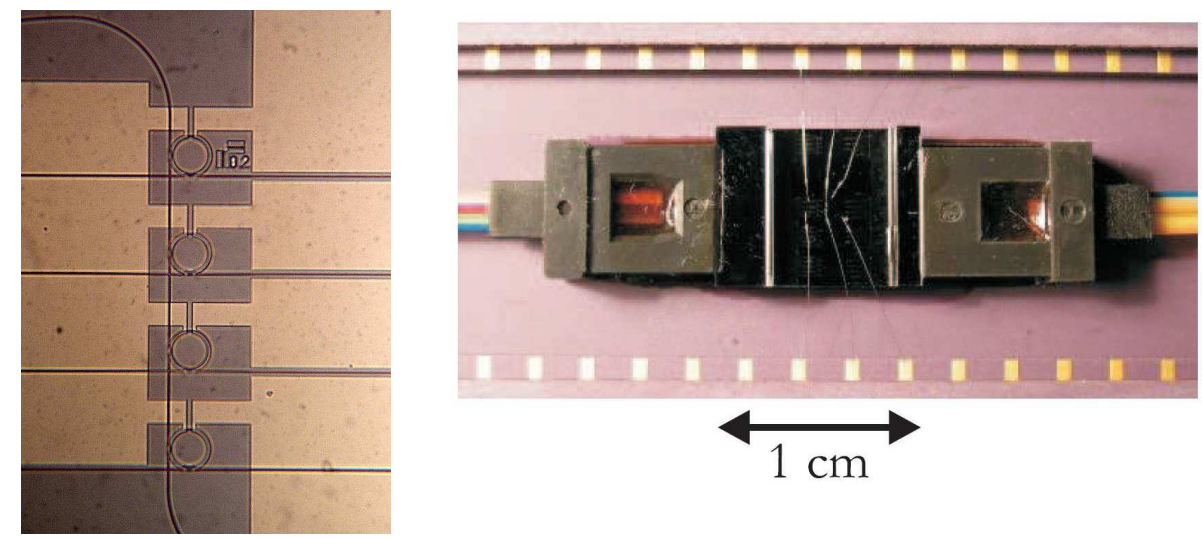

Figure 1.3: Microscope picture (left) and photograph (right) of an optical chip. The rings guide certain wavelength to different output fibers. The gold structures are used to heat up the rings, allowing to dynamically change the wavelengths they transmit. Image source PhD Thesis E.J. Klein 2007 [56].

such chips, functionality is created by manipulating light on a micro- or nanoscale. Light is guided in tiny dielectric or semiconductor waveguides [101], [88], [71], [12], [75], much like in optical fibers. Coming from a laser or from an optical fiber, the light propagates along the channels on the surface of the optical chip. This guidance and well-chosen layout of waveguides provide passive functionality - e.g. splitting the power or filtering the light - and furthermore, the guides may be used to transport the light to the areas of the chip where interaction with the outside world takes place. The two main areas of application of optical chips are sensors [8] and telecommunication.

\subsubsection{Telecommunication}

At the ends of the optical fibers used in telecommunication networks, integrated optical components may be used to modulate, combine or route signals. Their main application in the backbone network - the high-speed links between data centers or internet providers - is in so-called Wavelength Division Multiplexing (WDM). In WDM, different signals are transmitted at different wavelengths (colours) of light, as explained above. Many different data streams are encoded at different wavelengths, all of which are combined 


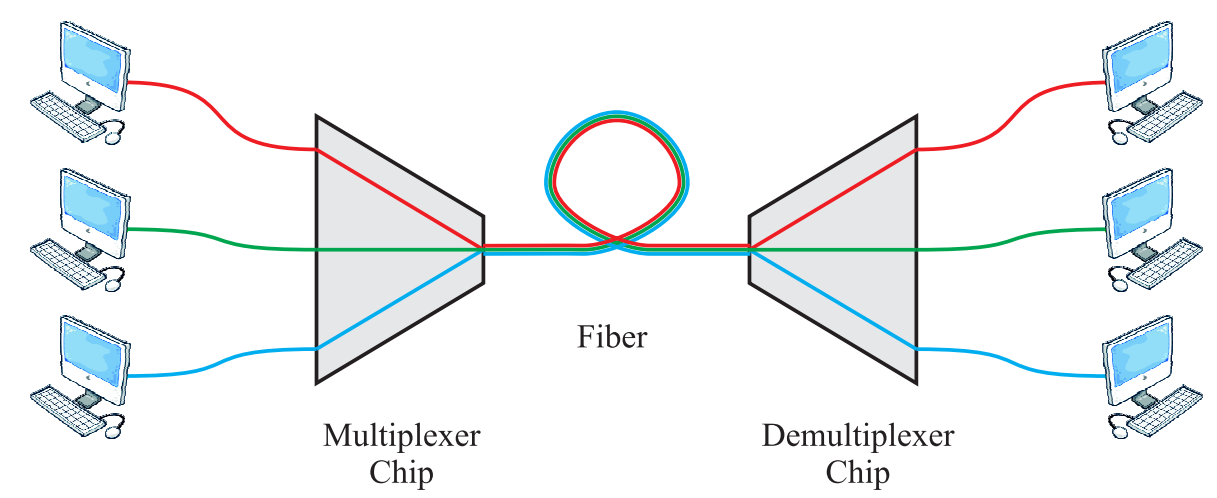

Figure 1.4: Schematic of Wavelength Division Multiplexing. Multiple colours of light, each carrying distinct data, are transmitted on the same optical fiber.

into one optical signal that is sent through the optical fiber (Figure 1.4). This combining, and, at the other end of the fiber, the demultiplexing of the signals, is done in integrated optical chips. These chips are structured to have a strongly wavelength dependent response, routing different wavelengths into different output channels or from different input channels into the same output channel.

Some examples of telecommunications building blocks, (pieces of) which will serve as examples in this thesis, are Arrayed Waveguide Gratings, MachZehnder interferometers, spot size converters, and photonic crystal based filters. In an Arrayed Waveguide Grating (Figure 1.5), the light is divided over many waveguides, which introduce a very well-defined wavelengthdependent phase difference. Due to this phase difference, the phase front experiences a wavelength-dependent tilt and light of different wavelengths gets focused on different output waveguides. If the device is used the other way around, with many input guides containing the correct wavelengths, all these input signals get focused on the same output waveguide. For the design of Arrayed Waveguide Gratings, one needs a very accurate determination of the propagation constant of the light in all waveguides.

A Mach-Zehnder interferometer (Figure 1.6) is a device in which the input light is split into two branches. One of the branches may be exposed to the environment, or be modified by an electrical voltage, which slows down or speeds up the light passing through it. At the position where the two branches are combined again, the difference between the two branches 


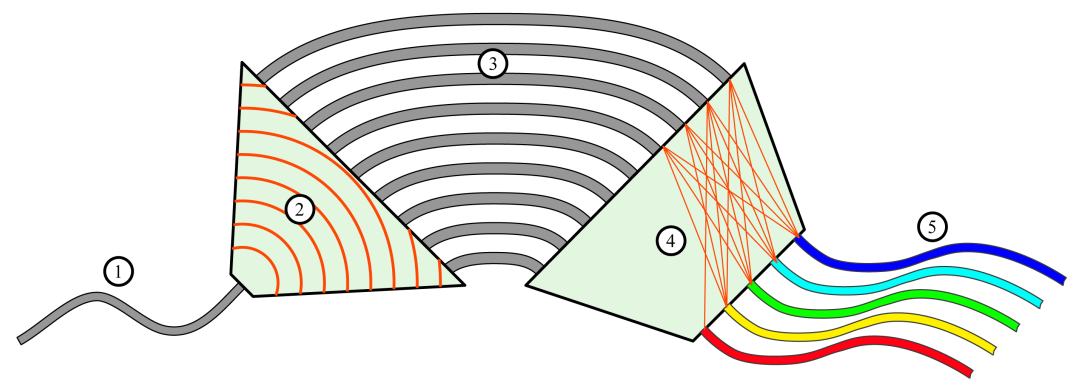

Figure 1.5: Arrayed Waveguide Grating: Incoming light (1) is divided (2) over the many central waveguides (3), causing a wavelength-dependent tilt at the output of those waveguides; thus different colours are focused in (4) on different output waveguides (5). Image source www.wikipedia.org, user Dr. Schorsch.

means that the two waves are not in phase anymore - which has a direct effect on the transmitted power. Mach-Zehnder interferometers may be used in both telecommunication - to modulate the intensity of a laser beam, for example - and in sensors, where the phase difference induced in one of the branches can be a measure of some chemical concentration. More on sensors follows later.

In many material systems, the waveguides that are most appropriate for the main function of the optical chip are not well suited for connection to an optical fiber. For example, in silicon nitride stripe waveguides one wants to be able to make sharp bends with minimal losses; these sharp bends are needed to minimize the total chip size. To this end, a relatively thick guide is needed. The size of the optical fields in such a high-contrast waveguide, however, is much smaller than in a standard optical fiber as used in telecommunication systems. Since the loss at the transition between the fiber and the waveguide depends on how closely alike the two fields are, they have to be matched as well as possible. The optical waveguide field can be expanded to match the fiber by slowly thinning ('tapering') the waveguide. Such structures that convert the field profile from one waveguide to another are called spot size converters.

Photonic crystals (see e.g. Figure 1.7) are structures in which the properties of the material vary periodically on length scales smaller than the wavelength of the light [51], [111], [81]. The behaviour of light in photonic 

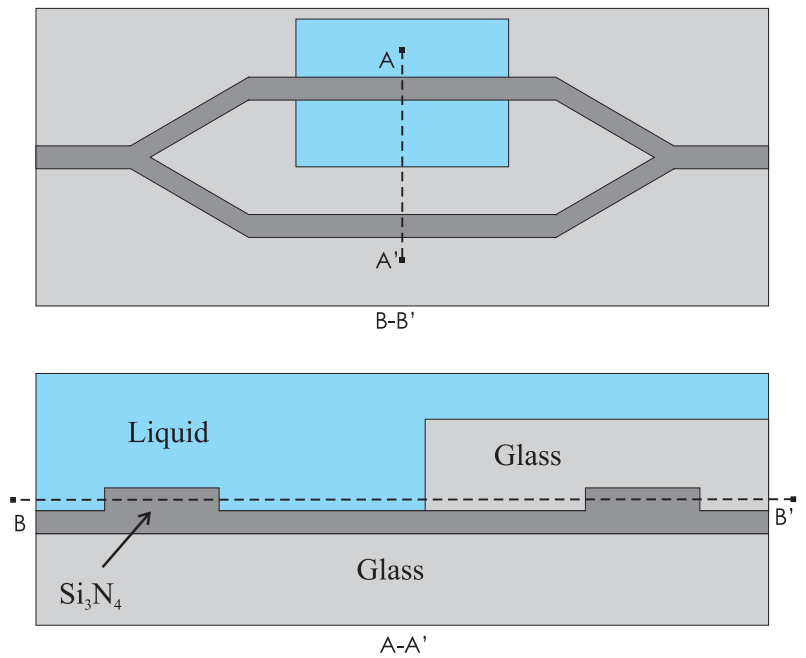

Figure 1.6: Mach-Zehnder interferometer with one arm exposed to a liquid. Changes in the refractive index of the liquid, caused by e.g. varying concentrations of sugar in water, only affect that arm, modifying the transmitted power of the interferometer.

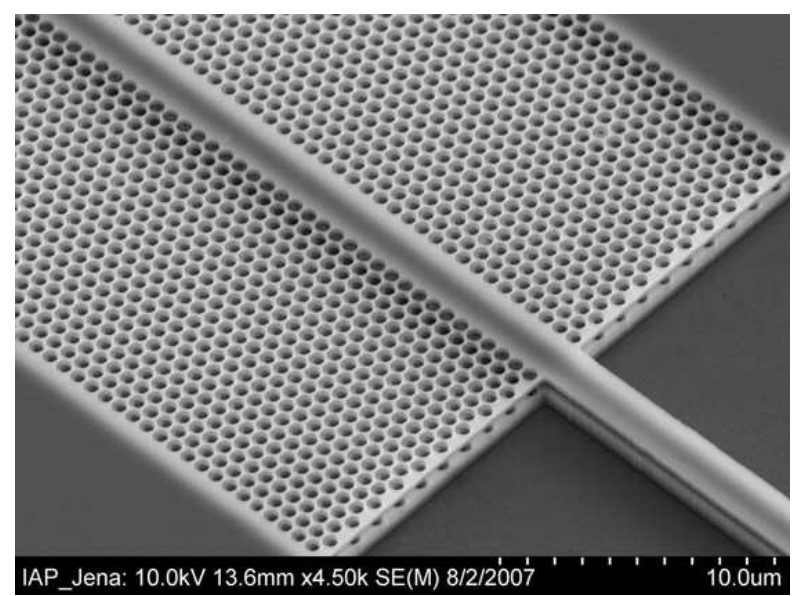

Figure 1.7: Photonic crystal waveguide: The light is guided in part due to the periodic arrangement of holes in the slab. The response of such devices can be strongly wavelength-dependent. Image source C. Helgert, Institute of Applied Physics, University of Jena, Germany. 
crystals is very strongly wavelength dependent; for some wavelengths, it is even impossible for the light to propagate through the crystal. The wavelength dependence of these structures is used for wavelength filtering and routing. Furthermore, photonic crystals tend to be very sensitive to external influences, and thus carry great potential for optical sensing.

\subsubsection{Sensors}

Integrated optical waveguides have the property that light propagating through them is mostly confined to the waveguide core with evanescent fields localized in the direct neighbourhood of the core. This makes light sensitive only to changes in materials very close to the waveguide core (see Figure 1.8). Minute quantities or very small concentrations of chemicals or biological antibodies can be accurately detected by integrated optical sensors (see Figure 1.9). Commercially available sensors can measure down to a refractive index change of about $10^{-8}$ [72], which corresponds to a sugar concentration of only about $0.7 \mathrm{mg}$ in a liter of water. One may use this localized sensitivity to probe tiny volumes of biological or environmental samples in so-called Lab on a Chip applications, which allow for fast and cheap diagnosis of diseases or constant online measurements of hazardous gases.

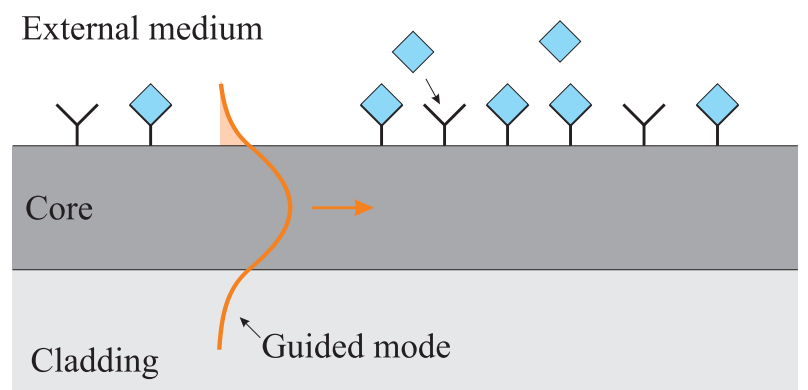

Figure 1.8: Biosensor waveguide structure: The propagation characteristics of the light in the waveguide are modified by antibodies that are captured by the receptors; only a very thin layer of material near the waveguide affects the light.

Furthermore, the high sensitivity of the response of guided light to external influences can also be used in telecommunication devices. As one 


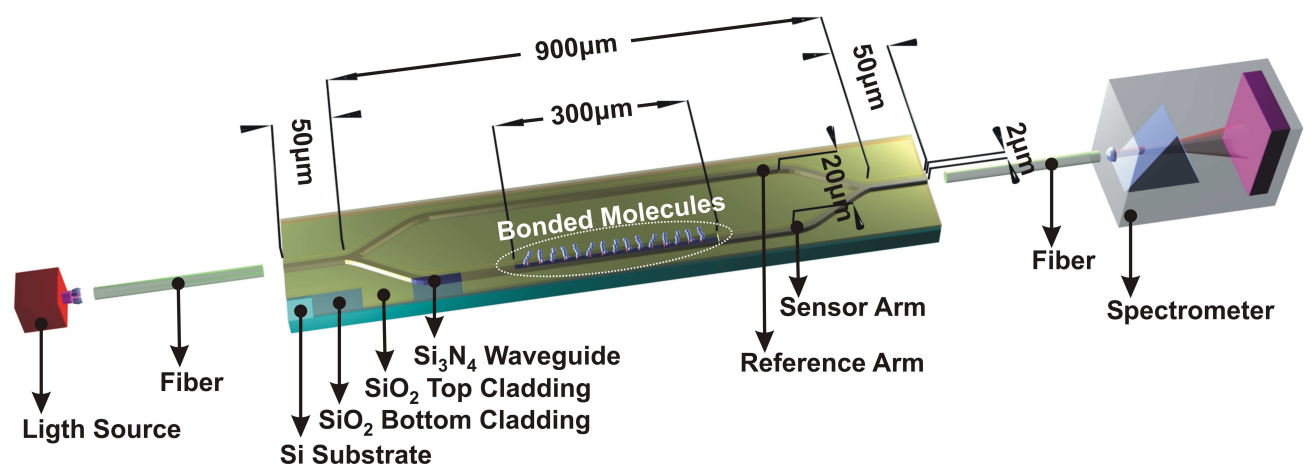

Figure 1.9: A broadband Mach-Zehnder biosensor [55]. Light with a wide spectrum is coupled into the chip. The light is split into two branches, one of which is exposed to water containing a certain concentration of specific molecules that can bond to the surface, modifying the propagation characteristics of the light. The difference between the two branches causes an intensity difference in the output light, which is analyzed by means of a spectrometer. Changes in the spectrum can be correlated with changes in the concentration.

example, guided light can interact with nanomechanically actuated cantilevers in order to switch or route the optical signal (Figure 1.13). Another example [73] shows how applying an external force to a photonic crystal, stretching it and thus modifying its period, significantly changing its wavelength response, light can be dynamically blocked or transmitted.

\subsubsection{Technology}

Optical chips are mostly fabricated [54] using technologies borrowed from the electronic IC industry. A flat substrate of e.g. silicon, indium phosphide, or fused silica glass is taken as a base, on which layers of materials with different optical properties are grown. These material layers are structured into the desired shapes by means of photolithography and etching steps; for an example process flow see Figure 1.10. Due to this fabrication technology, the light in optical chips is made to propagate mainly along the directions parallel to the initial substrate. 


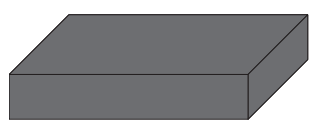

a)

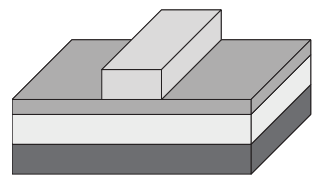

e)

Substrate

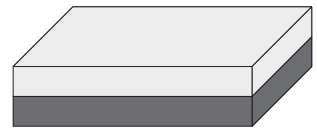

b)

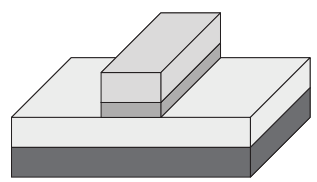

f)

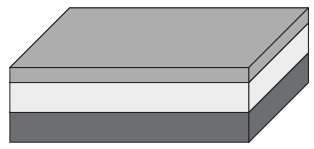

c)

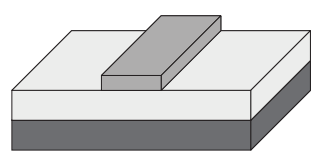

g)

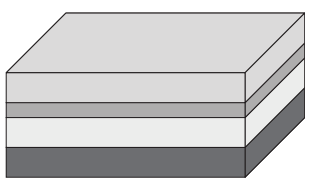

d)

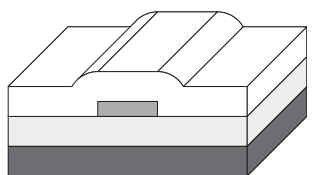

h)

Waveguide

Photoresist

$\square$ Buffer Cladding

Figure 1.10: Example fabrication steps for an optical waveguide. On top of a substrate wafer (a), a buffer layer (to optically isolate the waveguide from the substrate) is deposited or grown (b). A waveguide layer is deposited on top of the buffer (c), and a photoresist layer is deposited on top of it (d). After masking and exposing the photoresist, a development step (e) transfers the mask into the photoresist. Subsequently, an etching step defines the waveguides $(f)$. The photoresist is removed $(g)$, and the waveguide is covered with a cladding material ( $h$ ).

\subsection{Simulations}

In order to design optical chips, and especially to design devices that can be fabricated with reasonable yields, accurate but fast simulations [49], [99], [27] are of utmost importance.

However, unfortunately, in many cases the amount of calculation that needs to be performed to design a device has gone up faster than the increase in computer processor speeds can handle. Such an increase in necessary computational power is in part due to the increased complexity of the devices, in part due to the more advanced and accurate simulators, and in part due to the fact that fabrication data is at hand - and thus the effects of fabrication variations on the yield of a device can - and should be calculated. In fact, most actual devices are far too large - with small feature sizes - to simulate even one configuration at a time on a personal computer; one would require supercomputers. So the computational tools need to become smarter in order to be able to properly design a photonic device. 
This thesis describes simulation methods that attempt to decrease the computational effort by reducing the dimensionality of the calculations. In essence, this involves the use of a pre-defined set of basis functions in one spatial direction, and deriving equations in the other dimension(s) through a variational procedure.

This subsection will first show the Maxwell's equations and their specific form for the physical systems considered in this thesis; then, mode and scattering problems are described along with their mathematical formulations, and brief references to existing related alternative approaches. Finally, a short introduction into the main concepts of the work in this thesis will be given: the variational formalism and expansions into slab eigenmodes.

\subsubsection{Maxwell's equations}

The well-known macroscopic Maxwell's equations are a set of fundamental equations that describe the behaviour of electromagnetic fields 1 :

$$
\begin{aligned}
\nabla \times \mathbf{E}(x, y, z, t) & =-\frac{\partial \mathbf{B}}{\partial t}(x, y, z, t) \\
\nabla \times \mathbf{H}(x, y, z, t) & =\frac{\partial \mathbf{D}}{\partial t}(x, y, z, t)+\mathbf{J}(x, y, z, t) \\
\nabla \cdot \mathbf{D}(x, y, z, t) & =\rho(x, y, z, t) \\
\nabla \cdot \mathbf{B}(x, y, z, t) & =0
\end{aligned}
$$

where $\mathbf{E}$ is the electric field, $\mathbf{H}$ is the magnetic field, $\mathbf{D}$ is the dielectric displacement, and $\mathbf{B}$ is the magnetic induction. Finally, $\rho$ is the free charge density and $\mathbf{J}$ is the free current density.

The relations between $\mathbf{D}$ and $\mathbf{E}$, and between $\mathbf{B}$ and $\mathbf{H}$, are:

$$
\begin{aligned}
& \mathbf{D}(x, y, z, t)=\varepsilon_{0} \mathbf{E}(x, y, z, t)+\mathbf{P}(x, y, z, t) \\
& \mathbf{B}(x, y, z, t)=\mu_{0}(\mathbf{H}(x, y, z, t)+\mathbf{M}(x, y, z, t)),
\end{aligned}
$$

where $\mu_{0}$ is the magnetic permeability of vacuum, $\varepsilon_{0}$ is the electric permittivity of vacuum, $\mathbf{P}$ is the polarization and $\mathbf{M}$ is the magnetization. We specialize here to simulations where only linear, isotropic, nonmagnetic, and

\footnotetext{
${ }^{1}$ Cartesian coordinates $x, y, z$ will be used throughout this thesis. $t$ denotes the time variable. To avoid misunderstandings caused by the re-use of symbols, for this chapter we write out all function arguments explicitly.
} 
lossless materials ${ }^{2}$ are present. Eqns. (1.5) and (1.6) then reduce to:

$$
\begin{aligned}
& \mathbf{D}(x, y, z, t)=\varepsilon_{0} \varepsilon(x, y, z) \mathbf{E}(x, y, z, t) \\
& \mathbf{B}(x, y, z, t)=\mu_{0} \mathbf{H}(x, y, z, t),
\end{aligned}
$$

where $\varepsilon$ is the spatially dependent relative electric permittivity.

If we now also restrict to cases where free currents and charges are absent, Maxwell's equations reduce to:

$$
\begin{aligned}
\nabla \times \mathbf{E}(x, y, z, t) & =-\mu_{0} \frac{\partial \mathbf{H}}{\partial t}(x, y, z, t), \\
\nabla \times \mathbf{H}(x, y, z, t) & =\varepsilon_{0} \varepsilon(x, y, z) \frac{\partial \mathbf{E}}{\partial t}(x, y, z, t), \\
\nabla \cdot\left(\varepsilon_{0} \varepsilon(x, y, z) \mathbf{E}(x, y, z, t)\right) & =0, \\
\nabla \cdot \mathbf{H}(x, y, z, t) & =0 .
\end{aligned}
$$

In this thesis we will work only in the frequency domain. All field components in Maxwell's equations are oscillating harmonically in time as $\exp (\mathrm{i} \omega t)$ with a single frequency $\omega$, usually specified by the vacuum wavelength $\lambda=\frac{2 \pi}{k}=\frac{2 \pi c}{\omega}$, for vacuum wavenumber $k$ and vacuum speed of light $c=\frac{1}{\sqrt{\varepsilon_{0} \mu_{0}}}$. The first two Maxwell's equations, now for $\mathbf{E}(x, y, z)$ and $\mathbf{H}(x, y, z)$, can then be written in a simplified form, using the common conventions for the complex notation for time harmonic fields:

$$
\begin{aligned}
\nabla \times \mathbf{E}(x, y, z) & =-\mathrm{i} \omega \mu_{0} \mathbf{H}(x, y, z), \\
\nabla \times \mathbf{H}(x, y, z) & =\mathrm{i} \omega \varepsilon_{0} \varepsilon(x, y, z) \mathbf{E}(x, y, z) .
\end{aligned}
$$

By taking the divergence of the curl equations (1.13) and (1.14) one derives the other two divergence equations (1.11) and (1.12), which are usually referred to as complementary Maxwell's equations. Therefore, in the following it will be sufficient to consider only the curl equations.

\subsubsection{Scattering}

In scattering problems, one is interested in what happens to the light for a given input. For example, an optical fiber launches light into a waveguide of an optical chip - how does that light propagate along the chip, how much power ends up in which other waveguides, and how much is reflected back toward the fiber?

\footnotetext{
${ }^{2}$ Note, however that most of the theory also could be applied to more general situations.
} 

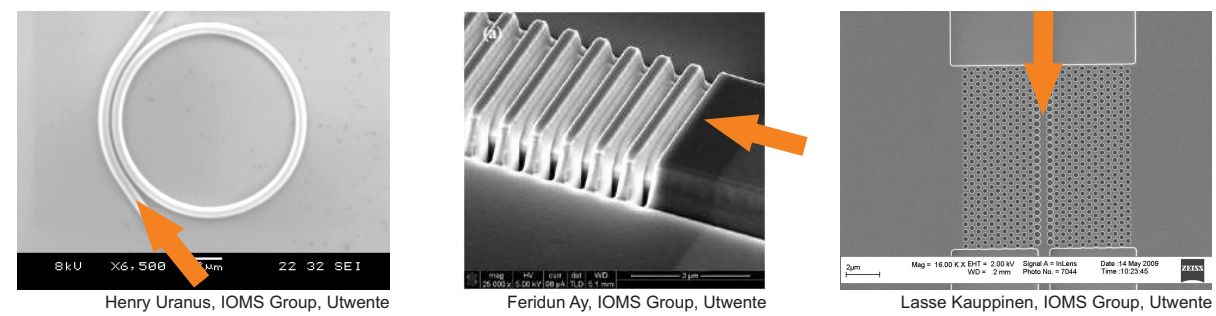

Figure 1.11: Examples of structures for which scattering simulations are necessary; light comes in from the direction of the arrows, and one wants to know where it goes. From left to right: A microring resonator, which exhibits strongly wavelength-dependent phase shifts of the light; a grating, which selectively reflects certain wavelength ranges; and a photonic crystal waveguide.

Scattering simulations can be performed in either the time domain or in the frequency domain. In the time domain, the full Maxwell's equations need to be solved. The most-used method for this is the so-called Finite Difference Time Domain method [97]. The great advantage of time domain calculations is that one may Fourier transform the results in order to obtain a full spectral response in one run. A disadvantage, however, is that the calculations tend to be very slow and memory-consuming. In the frequency domain, one calculates the response of the system to one single frequency of light. Usually, this can be done faster than time domain simulations but of course, for each frequency of interest, one needs to do a separate calculation. This thesis deals only with frequency domain simulations.

For 3D vectorial scattering problems the full frequency domain Maxwell's equations (1.13), (1.14) have to be solved. For 2D scattering problems, in which neither the fields nor the structure depend on $z$, the principal component of the transversal electric (TE) field, $E_{z}$, satisfies the equation

$$
\Delta E_{z}(x, y)+k^{2} \varepsilon(x, y) E_{z}(x, y)=0,
$$

while the principal component $H_{z}$, of the transversal magnetic (TM) field, is the solution of

$$
\nabla \cdot\left(\frac{1}{\varepsilon(x, y)} \nabla H_{z}(x, y)\right)+k^{2} H_{z}(x, y)=0 .
$$




\section{Scattering Solvers}

Simulations of scattering problems can be classified in three main categories: Unidirectional, bidirectional, and omnidirectional methods.

\section{Unidirectional methods}

In many applications, the flow of light is designed to run mainly in one direction; reflections are assumed to be insignificant, and the angle of the propagation of the light with respect to the 'simulation direction' is not very large. Under these assumptions, a unidirectional approximation of the Maxwell's equations may be used, in which the problem becomes an initial value problem; given the field at one end of the calculation window, the algorithms propagate this field to the other side in one go. This simplification to a unidirectional problem greatly decreases the amount of computational effort that is needed. However, of course, it can only be applied to a limited class of problems. The most important example of unidirectional simulation methods is the Beam Propagation Method [57], [63].

\section{Bidirectional methods}

In devices in which reflections are important, but the light is still expected to mainly travel along two opposite directions, bidirectional algorithms may be applied. In many components, reflections happen at a limited number of discrete places in the design. Bidirectional algorithms can take advantage of this limited number by performing two separate unidirectional - counterpropagating - simulations everywhere, except at the reflection points, where the two simulations couple. Examples of bidirectional methods are the Bidirectional Beam Propagation Method [63], the Bidirectional Eigenmode Propagation method [105], [26], and the Method of Lines [77].

\section{Omnidirectional methods}

In true omnidirectional methods, there is no preferred direction of the light. In principle, it may enter from and exit in any direction, which means that the boundaries of the simulations have to be be made transparent for outgoing light while still allowing influx to be prescribed. Popular examples are general purpose numerical approaches, like Finite Element methods, Finite Difference Frequency and Time Domain methods [97], Eigenmode Expansion methods [33] and Green's Function methods [74]. 


\subsubsection{Mode solving}

One of the most common tasks in the design of components is to assess how light propagates in a straight waveguide. In a straight waveguide, guided modes may exist which propagate losslessly with a certain propagation constant along the guide. The propagation constant of the modes is an extremely important quantity to calculate, since it directly influences the phase of light passing through the waveguide. Beside the propagation constant, the field profile of a mode (see e.g. Figure 1.12) is also often needed, since it can be used to estimate how strongly the light's phase and amplitude are modified by external influences, and also to determine the approximate losses in abrupt transitions, such as fiber-to-chip couplers.
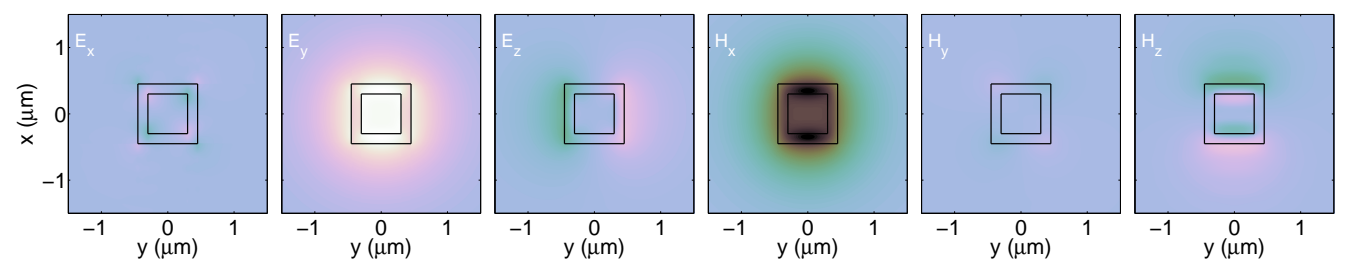

Figure 1.12: All six electromagnetic field components of the fundamental mode of a box-shaped waveguide.

A waveguide is a system that is uniform along one spatial direction. If $x, y, z$ are Cartesian coordinates with the $z$-axis parallel to the waveguide axis, such a structure is completely characterized by the transversal permittivity distribution $\varepsilon(x, y)$. In these structures there exist transverse field profiles that propagate along the $z$-axis with propagation constant $\beta$, i.e. for solutions of the form

$$
\mathbf{E}(x, y, z)=\mathbf{E}(x, y) \mathrm{e}^{-\mathrm{i} \beta z}, \quad \mathbf{H}(x, y, z)=\mathbf{H}(x, y) \mathrm{e}^{-\mathrm{i} \beta z} .
$$

When $\beta$ is real, the only $z$-dependent field variation is a phase factor, while the field amplitude remains constant. For complex values of $\beta$, the amplitude increases or decreases exponentially, but the shape of the field is maintained.

Consequently, the Maxwell's equations (1.13), (1.14) for the mode profile components $\mathbf{E}(x, y)$ and $\mathbf{H}(x, y)$ from (1.17) can be written as follows

$$
\begin{aligned}
\omega \mu_{0} \mathbf{H}(x, y)-\mathrm{i} C \mathbf{E}(x, y) & =-\beta R \mathbf{E}(x, y), \\
\omega \varepsilon_{0} \varepsilon(x, y) \mathbf{E}(x, y)+\mathrm{i} C \mathbf{H}(x, y) & =\beta R \mathbf{H}(x, y),
\end{aligned}
$$


with matrices

$$
R=\left(\begin{array}{ccc}
0 & 1 & 0 \\
-1 & 0 & 0 \\
0 & 0 & 0
\end{array}\right), \quad C=\left(\begin{array}{ccc}
0 & 0 & \partial_{y} \\
0 & 0 & -\partial_{x} \\
-\partial_{y} & \partial_{x} & 0
\end{array}\right)
$$

Solving the mode equation thus reduces to finding combinations of the transverse field profile $\mathbf{E}(x, y), \mathbf{H}(x, y)$ and the propagation constant $\beta$.

If the index contrast is low, implying that certain derivatives of the index can be neglected, the following scalar mode equations can be derived: The principal component of transversal electric (TE) modes, $E_{y}$, satisfies the equation

$$
\Delta E_{y}(x, y)+k^{2} \varepsilon(x, y) E_{y}(x, y)=\beta^{2} E_{y}(x, y),
$$

while for TM polarized modes the principal magnetic field component is governed by the equation:

$$
\nabla \cdot\left(\frac{1}{\varepsilon(x, y)} \nabla H_{y}(x, y)\right)+k^{2} H_{y}(x, y)=\beta^{2} \frac{1}{\varepsilon(x, y)} H_{y}(x, y) .
$$

If one restricts the mode problem (1.18), (1.19) to systems that do not have any $y$-dependence, and in which, furthermore, also the solutions do not depend on $y$, the resulting equations describe two one-dimensional mode equations; the two polarizations become completely decoupled: TE polarized modes are described by the eqn.

$$
E_{y}^{\prime \prime}(x)+k^{2} \varepsilon(x) E_{y}(x)=\beta^{2} E_{y}(x),
$$

while TM modes are solutions of

$$
\left(\frac{1}{\varepsilon(x)} H_{y}^{\prime}(x)\right)^{\prime}+k^{2} H_{y}(x)=\beta^{2} \frac{1}{\varepsilon(x)} H_{y}(x) .
$$

Over the years, many algorithms for mode solving have been developed, ranging from solvers for one-dimensional multilayer waveguides (1D mode solvers) to advanced fully-vectorial solvers for two-dimensional crosssections.

An example of a 1D mode solving algorithm is the Transfer Matrix Method [38], which is very efficient for waveguides with piecewise constant permittivity. Another option is to use a numerical discretization like the Finite Element Method, which is especially advantageous if the permittivity 
profile of the waveguide is not piecewise constant or if the coefficients in the mode equations are not real-valued such that propagation constants have to be identified in the complex plane rather than on the real axis.

For 2D cross-sections, major examples of mode solvers are purely numerical methods like the Finite Difference, Finite Element solvers [50] or the Method of Lines [77], mode expansion methods like the Film Mode Matching Methods [95], boundary methods like the Boundary Element Method and the Wave Matching Method [60], the Spectral Index Method [67], and more approximate methods like the Effective Index Method [101], [64], [2]. All these methods have their own strengths and weaknesses with respect to speed, memory use, accuracy, and applicability. More on this subject can be found in [101], [53], [59], [82], [102] and [16].

\subsubsection{Effective Index Method}

As stated above, propagation of light in optical chips happens mainly in the plane parallel to the substrate. Because of these preferred directions, a lot of simulation methods treat only those directions, and approximate the solution in the other direction in some way. The common way to do this is by means of the Effective Index Method. One calculates the 1D modes of each vertical cross-section by means of a one-dimensional mode solver, and uses the propagation constants of these modes in the equations to be solved for the remaining two dimensions. This procedure is most suitable for frequency domain simulations; the modes of the cross-sections will be different for all wavelengths, so in time domain simulations, one would need to take this into account by having frequency-dependent coefficients [90]. However, if one is interested only in a small wavelength range, the approximation of constant coefficients (using e.g. the cross-section mode of the wavelength in the middle of the interval of interest) can still be good enough.

\subsubsection{Variational formulation}

Instead of solving the partial differential equations in a direct way, one may also choose to find the critical points of a functional [101], [31]. Such a critical point is defined as a solution for which the variational derivative of the functional with respect to all unknowns vanishes. In principle, this search for critical points should be over all functions that satisfy the continuity and boundary conditions of the problem. However, the great advantage of 
working with a variational formalism is that one can restrict the function space over which to optimize - approximating the solution by a more limited set of functions. The work in this thesis makes heavy use of this formalism.

For the 3D vectorial scattering problem (1.13), (1.14), the following functional is used:

$\mathcal{F}(\mathbf{E}, \mathbf{H})=\int\left(\mathbf{E} \cdot(\nabla \times \mathbf{H})+\mathbf{H} \cdot(\nabla \times \mathbf{E})-\mathrm{i} \omega \varepsilon_{0} \varepsilon \mathbf{E}^{2}+\mathrm{i} \omega \mu_{0} \mu \mathbf{H}^{2}\right) d x d y d z$,

while the simpler functionals

$$
\mathcal{F}\left(E_{y}\right)=\int\left[-\left|\nabla E_{y}(x, y)\right|^{2}+k^{2} \varepsilon(x, y) E_{y}^{2}(x, y)\right] d x d y
$$

for TE polarized light and

$$
\mathcal{F}\left(H_{y}\right)=\int\left[-\frac{1}{\varepsilon(x, y)}\left|\nabla H_{y}(x, y)\right|^{2}+k^{2} H_{y}^{2}(x, y)\right] d x d y
$$

for TM polarized light, correspondingly, cover the 2D scattering problems (1.15) and (1.16).

Solutions of the vectorial mode equations (1.18), (1.19) are critical points of the functional

$$
\mathcal{F}(\mathbf{E}, \mathbf{H})=\frac{\omega \varepsilon_{0}\langle\mathbf{E}, \varepsilon \mathbf{E}\rangle+\omega \mu_{0}\langle\mathbf{H}, \mathbf{H}\rangle+\mathrm{i}\langle\mathbf{E}, C \mathbf{H}\rangle-\mathrm{i}\langle\mathbf{H}, C \mathbf{E}\rangle}{\langle\mathbf{E}, R \mathbf{H}\rangle-\langle\mathbf{H}, R \mathbf{E}\rangle},
$$

with inner product $\langle\mathbf{A}, \mathbf{B}\rangle=\int \mathbf{A}^{*}(x, y) \cdot \mathbf{B}(x, y) d x d y$.

For the scalar mode equations (1.21), (1.22), we will use functionals

$$
\mathcal{F}\left(E_{y}\right)=\frac{\int\left[-\left|\nabla E_{y}(x, y)\right|^{2}+k^{2} \varepsilon(x, y) E_{y}^{2}(x, y)\right] d x d y}{\int E_{y}^{2}(x, y) d x d y}
$$

for TE polarized light and

$$
\mathcal{F}\left(H_{y}\right)=\frac{\int\left[-\frac{1}{\varepsilon(x, y)}\left|\nabla H_{y}(x, y)\right|^{2}+k^{2} H_{y}^{2}(x, y)\right] d x d y}{\int \frac{1}{\varepsilon(x, y)} H_{y}^{2}(x, y) d x d y} .
$$

for TM polarized light.

For each of the functionals (1.28), (1.29) and (1.30) the value of the functional at a critical point is equal to the propagation constant $\beta$. 


\subsubsection{Expansion into slab eigenmodes}

Since the variational formulation allows to restrict the space of functions over which to optimize the solution, the question arises which space of functions is a good choice. In this thesis, like in several other methods, we make use of the fact that most optical chips are fabricated from stacks of flat layers of some materials. In a uniform layer stack, the solution can be constructed by means of a superposition of rotated modal solutions of the one-dimensional mode equation (1.23) or (1.24). Thus, it seems natural to use those 1D modal solutions as a basis over which to expand the field in the vertical direction. Most mode expansion methods utilize a different set of 1D modes in each distinct cross-section, attempting to satisfy continuity conditions at the interfaces between cross-sections by matching the amplitudes of the modes on the two sides of an interface appropriately [4], [95]. Since in general, incomplete sets of modes in two cross-sections cannot be exactly expressed in each other, the interface conditions are not fully satisfied.

The work presented in this thesis performs a different form of mode expansion: Instead of using a local set of modes at each distinct cross-section, one or more reference cross-sections are chosen, and their modes are used in the expansion of the field everywhere; see Table 1.1. This immediately provides continuity of the relevant quantities, even across interfaces between cross-sections.

The procedure allows to reduce the spatial dimensionality of the problem at hand by one.

\subsection{Outline of thesis}

This thesis describes research performed to reduce the dimensionality of simulations of optical chips. The main ideas, using a variational formulation combined with mode expansion methods, are applied to both mode solving and scattering problems.

First, in Chapter 2, the method is implemented for the scalar mode problem. Then, the theory is expanded to vectorial mode problems in Chapter 3. In Chapter 4, two-dimensional scattering problems are dealt with. Chapter 5 shows full vectorial three-dimensional simulations, performed using one or more 1D modes in the expansion. Finally, Chapter 6 gives conclusions and a short outlook into possible future research. 

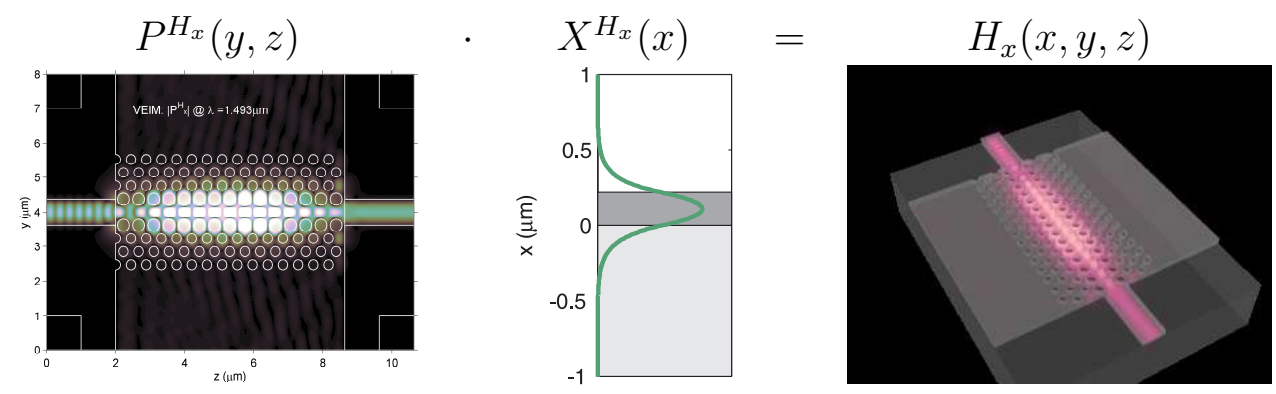

Table 1.1: Using mode expansion, the equations to be solved are one spatial dimension lower than in the original problem. For the scattering problem depicted here, one slab mode with profile $X^{H_{x}}$ is used; its coefficient $P^{H_{x}}$ is calculated in the plane. The total $3 D$ solution for $H_{x}$ may be found by multiplying the mode profile with its coefficient.

\subsection{Embedding}

This work has been carried out in the MESA+ Institute for Nanotechnology at the University of Twente in the framework of the NanoNed project TOE.7143 "Optical switching by NEMS-actuated resonator arrays, modelling and simulation tools", funded by the Dutch Technology foundation STW. The project was part of a cluster of three projects (TOE.7143, TOE.7144 and TOE.7145), which together aimed at "unfolding the potential of optical microresonators in photonic crystal structures promising higher density than is possible with current state of the art photonic integration". While colleagues in the more experimentally oriented projects investigated the design and realization of the mechanical (TOE.7144) and optical parts (TOE.7145) of the envisioned NanoElectroMechanical Systems (NEMS) for influencing optical channels, our project was concerned with theoretical aspects of externally perturbed optical microcavities, and with the development of computational modelling and simulation tools.

\subsection{Publications}

Work on this thesis was accompanied by the following publications in international refereed journals. The material of these papers constitutes the basis for the chapters of the thesis. 

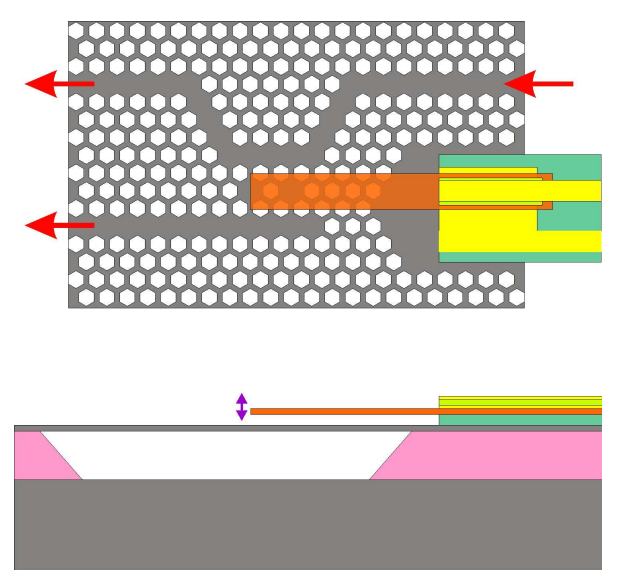

Figure 1.13: Design of a nanomechanically actuated optical switch. The cantilever perturbs the optical field in the photonic crystal cavity, in order to switch light from one output channel to another. Image source MESA+ Institute for Nanotechnology, University of Twente, The Netherlands.

- O. V. Ivanova, M. Hammer, R. Stoffer, and E. van Groesen. A variational mode expansion mode solver. Optical and Quantum Electronics, 39(10-11):849-864, 2007 [Chapter 2]

- M. Hammer and O. V. Ivanova. Effective index approximations of photonic crystal slabs: a 2-to-1-D assessment. Optical and Quantum Electronics, 2009. (available online, DOI 10.1007/s11082-009-9349-3)

- O. V. Ivanova, R. Stoffer, and M. Hammer. A variational mode solver for optical waveguides based on quasi-analytical vectorial slab mode expansion. Optics Communication, 2009. (submitted) [Chapter 3]

- O. V. Ivanova, R. Stoffer, and M. Hammer. A dimensionality reduction technique for 2D scattering problems in photonics. Journal of Optics, 2010. (accepted) [Chapter 4]

- O. V. Ivanova, R. Stoffer, and M. Hammer. A dimensionality reduction technique for $3 \mathrm{D}$ vectorial scattering problems in photonics. Journal of Lightwave Technology, 2010. (in preparation) [Chapter 5]

This work was also presented at a number of (international) conferences: 
- O. V. Ivanova, M. Hammer, R. Stoffer, and E. van Groesen. Variational effective index mode solver. In Proc. of the 11th Annual IEEE/LEOS Benelux Chapter Symposium, Eindhoven, The Netherlands, 2006; In Proc. of MicroNed/NanoNed National Symposium, Eindhoven, The Netherlands, 2006

- O. V. Ivanova, R. Stoffer, M. Hammer, and E. van Groesen. A variational vectorial mode solver. In Proc. of the 17th International Workshop on Optical Waveguide Theory and Numerical Modelling (OWTNM), Eindhoven, The Netherlands, 2008

- O. V. Ivanova, R. Stoffer, M. Hammer, and E. van Groesen. A vectorial variational mode solver and its application to piecewise constant and diffused waveguides. In Proc. of the 12th International Conference on Mathematical Methods in Electromagnetic Theory (MMET), Odessa, Ukraine, 2008

- O. V. Ivanova, R. Stoffer, and M. Hammer. A dimensionality reduction technique for scattering problems in photonics. In Proc. of 1st International Workshop on Theoretical and Computational NanoPhotonics (TaCoNa-Photonics), Bad Honnef, Germany, 2008

- O. V. Ivanova, R. Stoffer, and M. Hammer. Dimensionality reduction in computational photonics through variational mode expansion. In Proc. of MicroNano Conference, Ede, The Netherlands, 2008

- M. Hammer and O. V. Ivanova. On effective index approximations of photonic crystal slabs. In Proc. of the 13th Annual Symposium IEEE/LEOS Benelux Chapter, Enschede, The Netherlands, 2008; In Proc. of 18th International Workshop on Optical Waveguide Theory and Numerical Modelling (OWTNM), Jena, Germany, 2009

- O. V. Ivanova, R. Stoffer, L. Kauppinen, and M. Hammer. Variational effective index method for 3D vectorial scattering problems in photonics: TE polarization. In Proc. of Progress In Electromagnetics Research Symposium (PIERS), Moscow, Russia, 2009; In Proc. of MicroNano Conference, Delft, The Netherlands, 2009; In Proc. of MESA+ Symposium, Enschede, The Netherlands, 2009 
- R. Stoffer, O. V. Ivanova, M. Hammer, and H. J. W. M. Hoekstra. A guided mode view on Near-field Scanning Optical Microscopy measurements of optical magnetic fields with slit probes. In Proc. of the 19th International Workshop on Optical Waveguide Theory and Numerical Modelling (OWTNM), Cambridge, UK, 2010. (submitted)

We also contributed to a journal on precision engineering:

- R. Stoffer, O. V. Ivanova, and T. Korthorst. Smart algorithms and smart design tools. Mikroniek, 49(5):21-25, 2009 



\section{Scalar mode problems}

A variational approach for the scalar modal analysis of dielectric waveguides with arbitrary piecewise constant rectangular 2D cross-sections is developed. It is based on a representation of a mode profile as a superposition of modes of constituting slab waveguides times some unknown continuous coefficient functions, defined on the entire coordinate axis. The propagation constant and the lateral functions are found from a variational principle. It appears that this method with one or two modes in the expansion preserves the computational efficiency of the standard effective index method while providing more accurate estimates for propagation constants, as well as well-defined continuous approximations for mode profiles. By including a larger number of suitable trial fields, the present approach can also serve as a technique for rigorous semivectorial mode analysis.

\subsection{Introduction}

A variety of methods has been developed for the modal analysis of dielectric waveguides. References [16], [102], [82] present a detailed overview of the techniques. Among these, one of the most popular approximate approaches is the Effective Index Method (EIM) [64]. While being rather intuitive and computationally very efficient, the inherent approximations limit the range of its applicability: problems that occur are e.g. undefined effective indices in a slab region below cut-off and, as a result, only rather heuristically defined field profiles. Several methods exist that in certain respects might be viewed as improvements of the EIM; we mention the (Film) Mode Matching 
Method (MMM) [76] [95], in which the total field is expanded in the terms of the local slab modes and later matched across the interfaces, the Spectral Index Method [103], in which the total field is put to zero at certain distances from the interfaces with strong refractive index contrast and later expanded in Fourier series, the Weighted Index Method [80], which using a variational procedure finds the modal field profile as the best separable solution of the wave equation, another technique which corrects the Effective index Method (CEIM) [100] and is based on a linear combination of three approximations, and finally the Rigorous Effective Index Method [2], which, for rib waveguides with outer slices above cut-off, by variational means finds a simple transcendental equation for the propagation constant.

In this chapter we propose a Variational Mode Expansion Method (VMEM), which uses modes of the constituting waveguides in the representation of the field not only in the corresponding slab, but in the whole waveguide. On the basis of these field templates, approximations for guided modes are then derived by consistently applying a variational restriction procedure. A characteristic feature of this method is that by using continuous field templates the mode field profiles that are found are automatically continuous.

On the one hand, if one or two modes are used in the expansion this method can be viewed as an alternative approach for improvement of the EIM, preserving its computational efficiency. On the other hand, by including a large number of suitable basis fields, the present approach can also serve as a technique for rigorous semivectorial mode analysis. In that case the MMM (restricted to scalar/semivectorial calculations) and the present VMEM appear to be quite similar, because both methods are based on expansion into slab modes. Still there are several differences. First, in the MMM the eigenmodes of each slice are used for the representation of the field of only that particular slice, while in VMEM they also contribute to the solution on all other slices. Second, due to the specific field template the VMEM field profiles are automatically continuous (even with only one mode in the expansion), contrary to the MMM solutions. And third, the VMEM is rigorously derived from a variational principle, rather than by employing projection techniques as is commonly seen in the MMM. The variational background of the VMEM leads to certain properties of the solution, e.g. a monotonous convergence of the propagation constants.

The chapter is organized as follows. In the next section we put the problem of finding guided modes in a variational form. In section 2.3 the 
theory behind the VMEM is outlined. Section 2.3 .2 comments on the relation of the present approach with equations familiar from the "standard" EIM. Results of calculations for a series of waveguide structures including several benchmark examples are given in section 2.4. Finally, in section 2.5 conclusions are presented. In view of the fact that the formulation of the VMEM is similar for both TE and TM polarization, the general theory will be provided only for the former, while the equations which differ in the case of TM polarization will be given in the appendix.

\subsection{Variational form of the mode problem}

Consider a $z$-invariant dielectric waveguide given by a piecewise constant refractive index distribution $n(x, y)$ on its cross-section $\Omega$. We are searching for nontrivial solutions of the scalar TE mode equations for the dominant electric field component $E=E_{y}(x, y)$ :

$$
\Delta E+k^{2} n^{2}(x, y) E=\beta^{2} E, \quad(x, y) \in \Omega
$$

in the form of profiles, propagating in the $z$-direction with propagation constant $\beta$ at given vacuum wavelength $\lambda=2 \pi / k$.

There are two cases to be distinguished. On the one hand, if the computational window covers the entire cross-section plane $\Omega=\mathbb{R}^{2}$ a solution of Eq. (2.2.1) should be a continuous square integrable function on $\mathbb{R}^{2}$. On the other hand, for a rigorous analysis, the computational window can be restricted to be bounded in the $x$-direction, $x \in\left[x_{0}, x_{1}\right]$, while it remains to be unbounded in the $y$-direction. In this case the nullity on the boundaries $x=x_{0}$ and $x=x_{1}$ of a solution of Eq. (2.2.1) is required, so $x_{0}$ and $x_{1}$ should be far enough away from the structure to not influence the results significantly.

Solutions of (2.2.1) can also be formally found as critical points of the functional

$$
\mathcal{F}(E)=\frac{\int\left[-|\nabla E(x, y)|^{2}+k^{2} n^{2}(x, y) E^{2}(x, y)\right] d x d y}{\int E^{2}(x, y) d x d y} .
$$

The values of this functional at stationary points are equal to the propagation constants squared $\beta^{2}$. In case $\Omega$ is bounded in $x$-direction the critical 
points should be searched in the set of functions that vanish on the boundary; this is included as an extra condition.

The variational viewpoint permits a few direct conclusions concerning the influence of the computational window and the sets of trial functions [12]. Namely, let $\beta_{s}^{2}$ be the highest critical value of the functional (2.2.2) over the set of all functions $\mathcal{C}^{0}\left(\Omega_{s}\right)$, continuous functions defined on the computational domain $\Omega_{s}$ with zero on its boundary. Then by increasing the computational domain $\Omega_{b} \supset \Omega_{s}$ and taking the corresponding set $\mathcal{C}^{0}\left(\Omega_{b}\right) \supset \mathcal{C}^{0}\left(\Omega_{s}\right)$, the highest critical value $\beta_{b}^{2}$, found from (2.2.2), can only increase: $\beta_{b}^{2} \geq \beta_{s}^{2}$. Consequently, with increasing computational domain, the approximation of the propagation constant of the fundamental mode will approach its exact value from below.

Next, let $\Omega$ be a fixed domain and let $\beta_{\Omega, \mathcal{E}_{1}}^{2}$ and $\beta_{\Omega, \mathcal{E}_{2}}^{2}$ be the principal critical values of the functional (2.2.2) considered over the sets of the functions $\mathcal{E}_{1}$ and $\mathcal{E}_{2}$ correspondingly, defined over domain $\Omega$. If $\mathcal{E}_{1} \subset \mathcal{E}_{2}$, then from the principle of eigenvalue comparison it follows that $\beta_{\Omega, \mathcal{E}_{1}}^{2} \leq \beta_{\Omega, \mathcal{E}_{2}}^{2}$. In other words, by increasing the number of trial functions on the same domain the approximation value for the propagation constant of the fundamental mode can only increase.

\subsection{Variational mode expansion method}

Upon a division of the waveguide cross-section into $r$-homogeneous slices $S_{1}, \ldots, S_{r}$ with refractive index distribution $n_{1}(x), \ldots, n_{r}(x)$, the principal field component $E=E_{y}(x, y)$ is represented as a superposition of TE modes $X_{i}(x)$ of the constituting slab waveguides, times some unknown continuous coefficient functions $Y_{i}(y)$ :

$$
E(x, y)=\sum_{i=1}^{N} X_{i}(x) Y_{i}(y) .
$$

Fig. 2.1 shows an example and introduces the Cartesian axes $x, y$.

Note that functions $Y_{i}(y)$ are defined on the whole $y$-axis, which implies that modes $X_{i}(x)$ are relevant for the field in the whole waveguide. In the section 2.3.1 we will explain the process of selecting modes $X_{i}(x)$, while here we describe the method of finding the coefficient functions. 


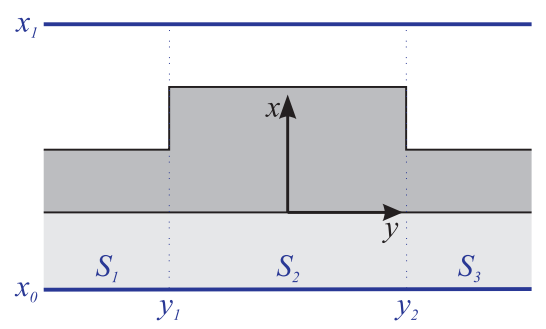

Figure 2.1: Sample geometry: rib waveguide. In this case the structure can be divided by vertical lines at $y_{1}, y_{2}$ into three slices $S_{1}, S_{2}, S_{3}$ with homogeneous refractive index distribution along the $y$-axis. As discussed in section 2.2, the mode problem will be considered on the entire $x$-axis, or, alternatively, on a computational window $x \in\left[x_{0}, x_{1}\right]$.

Restricting the functional (2.2.2) to the trial field (2.3.3) we arrive at the new problem of finding the critical points $\left\{Y_{1}, \ldots, Y_{N}\right\}$ of the functional

$$
\mathcal{F}\left(Y_{1}, \ldots, Y_{N}\right)=\frac{\int\left[-\left|\nabla\left(\sum_{i=1}^{N} X_{i}(x) Y_{i}(y)\right)\right|^{2}+k^{2} n^{2}(x, y)\left(\sum_{i=1}^{N} X_{i}(x) Y_{i}(y)\right)^{2}\right] d x d y}{\int\left(\sum_{i=1}^{N} X_{i}(x) Y_{i}(y)\right)^{2} d x d y} .
$$

Requiring this functional to become stationary leads to a vectorial differential equation, for the unknown function $\mathbf{Y}(y)=\left(Y_{1}(y), \ldots, Y_{N}(y)\right)$ with the propagation constant $\beta$ as a parameter, of the form

$$
\mathbf{F Y}^{\prime \prime}(y)+\mathbf{M}(y) \mathbf{Y}(y)=\beta^{2} \mathbf{F Y}(y)
$$

The matrices $\mathbf{F}$ and $\mathbf{M}$ are of dimension $N \times N$ and consist of elements

$$
\begin{gathered}
F_{g, h}=\int_{x_{0}}^{x_{1}} X_{g}(x) X_{h}(x) d x, \\
M_{g, h}(y)=\int_{x_{0}}^{x_{1}}\left(k^{2} n^{2}(x, y) X_{g}(x) X_{h}(x)-X_{g}^{\prime}(x) X_{h}^{\prime}(x)\right) d x .
\end{gathered}
$$

Note that inside each constituting slice the matrix $\mathbf{M}$ does not depend on $y$, so in the slice $S_{j}$ we can denote it as $\mathbf{M}^{(j)}$. 
Beyond (2.3.5), for a structure divided into slices, stationarity of (2.3.4) amounts to interface conditions of continuity of

$$
\mathbf{Y}(y) \quad \text { (as an essential condition) }
$$

and

$$
\mathbf{Y}^{\prime}(y) \quad \text { (as a natural condition). }
$$

Consequently the profiles $\mathbf{Y}$ now can be found by solving in the each separate slice $S_{j}$ the vectorial differential equation with constant coefficients:

$$
\left(\mathbf{Y}^{(j)}\right)^{\prime \prime}+\mathbf{T}^{(j)} \mathbf{Y}^{(j)}=\beta^{2} \mathbf{Y}^{(j)}, \quad \text { where } \mathbf{T}^{(j)}=\mathbf{F}^{-1} \mathbf{M}^{(j)}
$$

and matching the solutions across the interfaces according to the conditions (2.3.8) and (2.3.9).

Searching for solutions in the form of $\mathbf{Y}^{(j)}=\exp \left(\mu^{(j)} y\right) \mathbf{p}_{j}$, Eq. (2.3.10) requires that in the $j$-th slice the values $\mu^{(j)}$ and the vectors $\mathbf{p}^{(j)}$ satisfy the eigenvalue problem

$$
\mathbf{T}^{(j)} \mathbf{p}^{(j)}=\eta^{(j)} \mathbf{p}^{(j)}, \quad \text { with } \eta^{(j)}=\beta^{2}-\left(\mu^{(j)}\right)^{2}
$$

as eigenvalues and $\mathbf{p}^{(j)}$ as eigenvectors, which do not depend on $\beta$. Note that all the $\mu^{(j)}$ do. Since the matrix $\mathbf{T}^{(j)}$ is of dimension $N \times N$, solving the eigenvalue problem (2.3.11) yields $N$ eigenvalues $\eta_{1}^{(j)}, \ldots, \eta_{N}^{(j)}$ and corresponding eigenvectors $\mathbf{p}_{1}^{(j)}, \ldots, \mathbf{p}_{N}^{(j)}$. From the fact that the matrix $\mathbf{F}$ is symmetric positive definite and the matrix $\mathbf{M}^{(j)}$ is symmetric we can conclude that the matrix $\mathbf{T}^{(j)}=\mathbf{F}^{-1} \mathbf{M}^{(j)}$ has only real eigenvalues. Looking only for exponentially decaying functions towards $\pm \infty$, the solution of (2.3.10) will be

$$
\mathbf{Y}=\left\{\begin{aligned}
\mathbf{Y}^{(j)}=\sum_{i=1}^{N}\left(a_{i}^{(j)} \exp \left(\mu_{i}^{(j)} y\right)+b_{i}^{(j)} \exp \left(-\mu_{i}^{(j)} y\right)\right) \mathbf{p}_{i}^{(j)}, \quad j=2, \ldots, r-1 \\
\quad \text { (in the each inner slice) } \\
\mathbf{Y}^{(1)}=\sum_{i=1}^{N} d_{i} \exp \left(\mu_{i}^{(1)} y\right) \mathbf{p}_{i}^{(1)} \quad \text { (in the left outer slice) } \\
\mathbf{Y}^{(r)}=\sum_{i=1}^{N} c_{i} \exp \left(-\mu_{i}^{(r)} y\right) \mathbf{p}_{i}^{(r)} \quad \text { (in the right outer slice) }
\end{aligned}\right.
$$

where all $\mu_{i}^{(1)}$ and $\mu_{i}^{(r)}$ must be positive, while in the inner slices the $\mu_{i}^{(j)}$ can be complex conjugate pairs. To ensure the former, $\beta$ must be larger than the 
square root of the highest eigenvalue among $\eta_{1}^{(1)}, \ldots, \eta_{N}^{(1)}$ and $\eta_{1}^{(r)}, \ldots, \eta_{N}^{(r)}$, as it follows from (2.3.11). Moreover, to satisfy the continuity requirements (2.3.8), (2.3.9) across the interfaces, at least in one of the inner slices $\mathbf{Y}^{(j)}$ must have harmonic behavior, which implies that $\beta$ must be smaller than the square root of the highest eigenvalue among those which corresponds to the inner slices: $\eta_{1}^{(j)}, \ldots, \eta_{N}^{(j)}$. Thus an interval $\mathcal{I}_{\beta}$ for admissible values $\beta$ is found.

Still the coefficients $\mathbf{a}^{(j)}, \mathbf{b}^{(j)}, \mathbf{c}$ and $\mathbf{d}$ have to be determined (where to shorten the notation we put $\mathbf{a}=\left[a_{1}, \ldots, a_{N}\right]$ etc). If the interface between slice $S_{j}$ and $S_{j+1}$ is $y=y^{(j)}$, by using the interface conditions (2.3.8) and (2.3.9) the following system of equations arises:

$$
\begin{gathered}
\mathbf{Y}^{(j)}\left(y^{(j)}\right)=\mathbf{Y}^{(j+1)}\left(y^{(j)}\right), \\
\left(\mathbf{Y}^{(j)}\left(y^{(j)}\right)\right)^{\prime}=\left(\mathbf{Y}^{(j+1)}\left(y^{(j)}\right)\right)^{\prime}, \quad j=1, \ldots, r-1,
\end{gathered}
$$

or, in matrix form,

$$
\mathbf{V} \mathbf{f}=0
$$

with a vector of all unknown coefficients,

$$
\mathbf{f}=\left[\mathbf{d}, \mathbf{a}^{(2)}, \mathbf{b}^{(2)}, \ldots, \mathbf{a}^{(r-1)}, \mathbf{b}^{(r-1)}, \mathbf{c}\right]^{\top},
$$

and the matrix

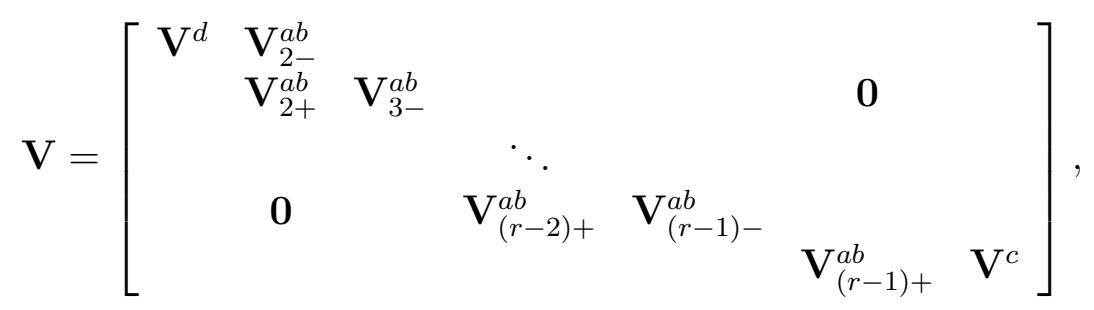

whose $s$-th line represents all the continuity conditions at the $s$-th interface. The columns of the submatrices can be written as:

$$
\begin{gathered}
\mathbf{V}^{d}(i)=\left[\begin{array}{c}
\exp \left(\mu_{i}^{(1)} y^{(1)}\right) \mathbf{p}_{i}^{(1)} \\
\mu_{i}^{(1)} \exp \left(\mu_{i}^{(1)} y^{(1)}\right) \mathbf{p}_{i}^{(1)}
\end{array}\right], \quad \mathbf{V}^{c}(i)=\left[\begin{array}{c}
-\exp \left(-\mu_{i}^{(r)} y^{(r-1)}\right) \mathbf{p}_{i}^{(r)} \\
\mu_{i}^{(r)} \exp \left(-\mu_{i}^{(r)} y^{(r-1)}\right) \mathbf{p}_{i}^{(r)}
\end{array}\right], \\
(2.3 .15) \\
\mathbf{V}_{j-}^{a b}=\left[\begin{array}{ll}
\mathbf{A}_{j-} & \mathbf{B}_{j-}
\end{array}\right], \quad \mathbf{V}_{j+}^{a b}=\left[\begin{array}{ll}
\mathbf{A}_{j+} & \mathbf{B}_{j+}
\end{array}\right],
\end{gathered}
$$


where

$\mathbf{A}_{j-}(i)=\left[\begin{array}{c}-\exp \left(\mu_{i}^{(j)} y^{(j-1)}\right) \mathbf{p}_{i}^{(j)} \\ -\mu_{i}^{(j)} \exp \left(\mu_{i}^{(j)} y^{(j-1)}\right) \mathbf{p}_{i}^{(j)}\end{array}\right], \quad \mathbf{B}_{j-}(i)=\left[\begin{array}{c}-\exp \left(-\mu_{i}^{(j)} y^{(j-1)}\right) \mathbf{p}_{i}^{(j)} \\ \mu_{i}^{(j)} \exp \left(-\mu_{i}^{(j)} y^{(j-1)}\right) \mathbf{p}_{i}^{(j)}\end{array}\right]$,

$$
\mathbf{A}_{j+}(i)=\left[\begin{array}{c}
\exp \left(\mu_{i}^{(j)} y^{(j)}\right) \mathbf{p}_{i}^{(j)} \\
\mu_{i}^{(j)} \exp \left(\mu_{i}^{(j)} y^{(j)}\right) \mathbf{p}_{i}^{(j)}
\end{array}\right], \quad \mathbf{B}_{j+}(i)=\left[\begin{array}{c}
\exp \left(-\mu_{i}^{(j)} y^{(j)}\right) \mathbf{p}_{i}^{(j)} \\
-\mu_{i}^{(j)} \exp \left(-\mu_{i}^{(j)} y^{(j)}\right) \mathbf{p}_{i}^{(j)}
\end{array}\right]
$$

for $i=1, \ldots, N, j=1, \ldots, r-1$. Propagation constants $\beta$ are thus those values from the interval $\mathcal{I}_{\beta}$ for which a nontrivial solution of Eq. (2.3.14) exists, i.e. those for which at least one eigenvalue of $\mathbf{V}$ becomes zero. Corresponding field profiles $E(x, y)$ can be found from Eq. (2.3.3).

\subsubsection{Modal basis functions}

Let the refractive index distribution in the waveguide within the $j$-th slice be $n_{j}(x)$. The corresponding modes are continuous solutions with continuous derivatives of

$$
X^{\prime \prime}(x)+k^{2} n_{j}^{2}(x) X(x)=\gamma^{2} X(x),
$$

with $\gamma$ as a propagation constant. There are two cases to be distinguished:

- If the computational window is unbounded, solutions of (2.3.18) must be square integrable. There is only a finite number of them.

- If the computational window in $x$-direction is $x \in\left[x_{0}, x_{1}\right]$, solutions of (2.3.18) are chosen to be zero at the boundary. There are infinitely many of them and, moreover, they form a complete discrete set of functions defined on $\left[x_{0}, x_{1}\right]$ with zero Dirichlet boundary conditions.

The detailed process of finding the solutions of Eq. (2.3.18) can be found e.g. in [101]. Ensuring positive-definiteness of the matrix $\mathbf{F}$ requires linear independence of the trial functions. This means that one should select which modes $X$ to take into expansion (2.3.3). Obviously a safe choice is to take a finite set of modes from a single slice. At the same time when taking into the expansion only a few modes, it is sometimes possible and, as our results show, beneficial to take also into account modes from other slices. 


\subsubsection{Relation with the Effective Index Method}

While the equations in section 2.3 appear rather involved, it is instructive to write them out for a case in which there is only one slab mode in the expansion. By taking only one term $E=X(x) Y(y)$ in the expansion (2.3.3) (with $X$ a mode profile of a reference slice; for the rib of Fig. 2.1 this would typically be the guided mode of the inner slice), according to (2.3.5) the corresponding equation for the function $Y$ is

$$
Y^{\prime \prime}(y)+\frac{\int_{x_{0}}^{x_{1}}\left(k^{2} n^{2}(x, y) X^{2}(x)-\left(X^{\prime}(x)\right)^{2}\right) d x}{\int_{x_{0}}^{x_{1}} X^{2}(x) d x} Y(y)=\beta^{2} Y(y) .
$$

As a slab waveguide mode profile, $X$ satisfies the equation

$$
X^{\prime \prime}(x)+k^{2} n_{\mathrm{r}}^{2}(x) X(x)=\gamma^{2} X(x)
$$

for propagation constant $\gamma$, where $n_{\mathrm{r}}$ is the refractive index profile of the reference slice. By inserting this equation into (2.3.19) and integrating by parts, we obtain, after defining the effective index $N_{\text {eff }}$ of the mode profile $X$ of the slice $\mathrm{r}$ through $\gamma=k N_{\text {eff: }}$ :

$$
Y^{\prime \prime}(y)+k^{2}\left(N_{\mathrm{eff}}^{2}+\frac{\int_{x_{0}}^{x_{1}}\left(n^{2}(x, y)-n_{\mathrm{r}}^{2}(x)\right) X^{2}(x) d x}{\int_{x_{0}}^{x_{1}} X^{2}(x) d x}\right) Y(y)=\beta^{2} Y(y) .
$$

Hence the equation for the lateral profile function $Y$ as it emerges from the variational procedure is similar to what is used in the EIM: in the reference slice one has $n(x, y)=n_{\mathrm{r}}(x)$, and the effective index appears to be the one corresponding to the mode profile $X$; in other slices this effective index is modified by the difference between the local permittivity and that of the reference slice, weighted by the local intensity of the reference mode profile. This means that even in slices in which there is no local mode above cut-off, a reasonable "effective index" can still be defined. Note that this recipe results directly from the application of the variational procedure; no heuristics are needed beyond the ansatz (2.3.3). 


\subsection{Numerical results and comparisons}

In short, the whole numerical procedure of mode finding can be outlined as follows: find the set of basis modes $X_{i}$; calculate matrices $\mathbf{T}_{j}$ and their eigenvectors and eigenvalues according to Eq. (2.3.11); determine the interval $\mathcal{I}_{\beta}$ for admissible values of $\beta$; identify values of $\beta$ from this interval, for which the matrix $\mathbf{V}$ from (2.3.14) has at least one zero eigenvalue; for each found propagation constant $\beta$ assemble the corresponding field profile according to Eq. (2.3.3). The validity of the method was checked for several waveguide structures. It should be mentioned that in all of the following examples our results agree remarkably well with rigorous numerical finite-element FEMLAB [22] solutions of the Eqs. (2.2.1) or (A-1).

\subsubsection{Rib waveguide}

A frequently investigated geometry for comparison of mode analysis methods [102], [60] is shown in Fig. 2.2(a). The structure can be divided into three slices $S_{1}, S_{2}, S_{3}$. Inside these slices the refractive index distribution is homogeneous along the $y$-axis. Since the first and last slices are equal, we will take into expansion (2.3.3) only modes from the first and second slices.

(a)

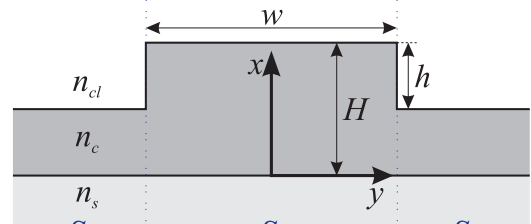

$S_{I}$

$S_{2}$

$S_{3}$ (b)

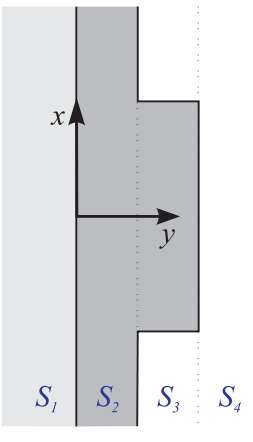

Figure 2.2: Cross-section of a benchmark rib waveguide. Geometrical parameters: $w=3 \mu \mathrm{m}$, varying $h, H=1 \mu \mathrm{m}$, refractive indices: $n_{s}=3.4$, $n_{c}=3.44, n_{c l}=1.0$, operating wavelength $\lambda=1.15 \mu \mathrm{m}$. (a) "Conventional" division into slices, computational window $x \in[-10,10] \mu m$; (b) an alternative division of the rotated structure.

In Fig. 2.3 propagation constants of the fundamental TE and TM modes, obtained by other methods, are compared to those of the VMEM. As was 
discussed in section 2.2 , with enlarging the mode set the propagation constant $\beta$ increases and thus approaches its exact value from below. Note that very accurate results compared to the rigorous finite element (FEMLAB) solutions can be obtained using only 16 modes in the expansion (2.3.3).
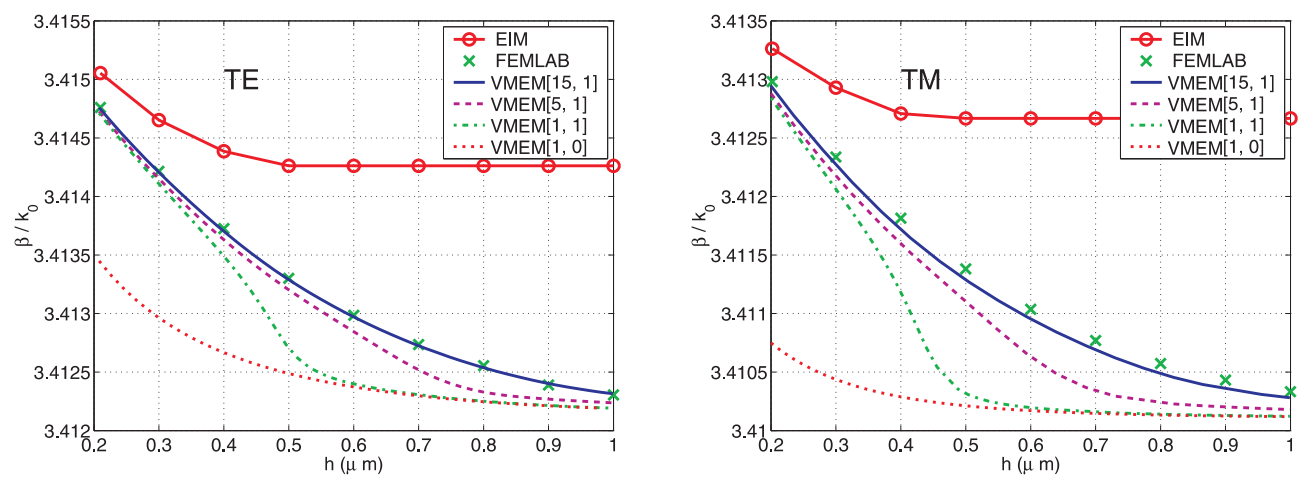

Figure 2.3: Effective indices $(\beta / k)$ of the fundamental TE and TM modes supported by the waveguide of Fig. 2.2(a) as a function of the rib height h. EIM: effective index method; FEMLAB: finite element [22] calculations on a mesh with about 20000 elements and a computational window $(x, y) \in$ $[-6.5,2.5] \times[-7.5,7.5] ; \operatorname{VMEM}[i, j]$ : the present method with $i$ and $j$ modes of slices $S_{2}$ and $S_{1}$ correspondingly, used in the expansion (2.3.3).

In Fig. 2.4 we show $X, Y$ and total field profiles of the fundamental TE modes for the VMEM[1,0], VMEM[1,1] and VMEM[5,1], for $h=0.4 \mu m$. From the $Y$ profiles one can see that in this structure the field in the middle of the inner slice is dominated by the fundamental mode of the inner slice, while in the outer slices the field is dominated by the fundamental mode of the outer slice. Around the interfaces between slices all considered modes play a significant role; apparently they are all needed to satisfy the interface conditions. From Figs. 2.3 and 2.4 it follows, that including the outer slice mode significantly improves the estimations of the field profiles as well as propagation constants, while the higher order modes of the inner slice mainly seem to smoothen the field around the interfaces.

A division into horizontal layers can be an alternative to the division of the waveguide cross-section described above, as is shown in the Fig. 2.2(b). There are four layers $S_{1}, S_{2}, S_{3}, S_{4}$ with homogeneous refractive index distribution along the $y$-axis. Effectively modes only from two layers $S_{3}$ and 
$S_{4}$ (alternatively, $S_{1}$ or $S_{2}$ ) are required.

(a)

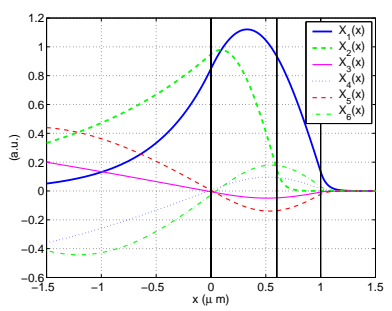

(b)

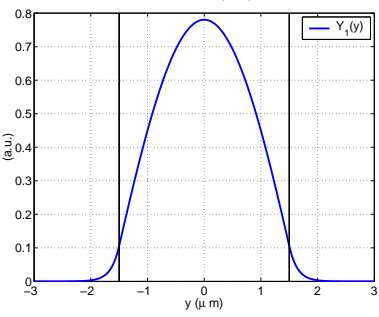

(c)

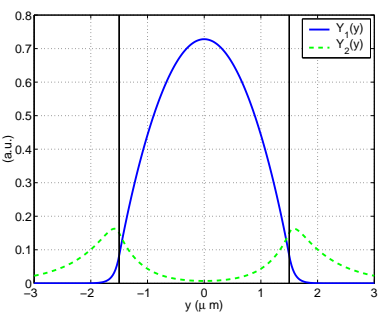

(d)

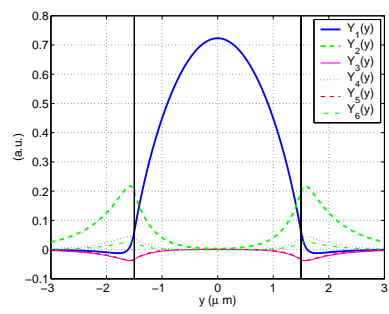

(b')

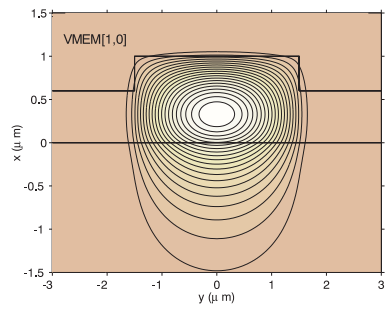

$\left(c^{\prime}\right)$

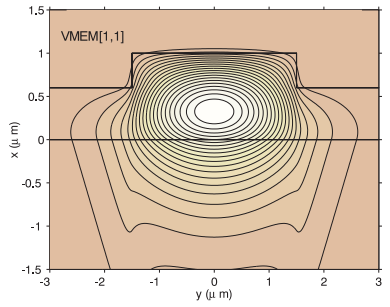

(d')

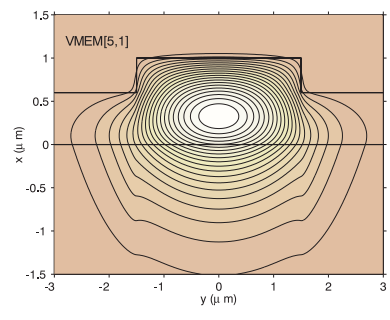

Figure 2.4: For the rib waveguide structure of Fig. 2.2(a) with $h=0.4 \mu \mathrm{m}$ : Plot (a) shows the TE mode profiles $X$ of the constituting slab waveguides: $X_{1}$ is the fundamental mode of the inner slice $S_{2} ; X_{2}$ is the fundamental mode of the outer slice $S_{1} ; X_{3}, X_{4}, X_{5}$ and $X_{6}$ - first, second, third and fourth order modes of the inner slice $S_{2}$ correspondingly. Plots (b), (c) and (d) show $Y$ profiles and plots (b'), (c') and (d') corresponding field profiles of the fundamental TE mode. (b) and ( $\left.b^{\prime}\right)$ correspond to VMEM[1,0], (c) and (c') to $\operatorname{VMEM}[1,1]$, and (d) and (d') to VMEM[5,1]. 
In Fig. 2.5 convergence of the effective index of the fundamental TE mode is shown. Note that for the symmetric structure only symmetric modes $X$ of slices $S_{3}$ and $S_{4}$ have an impact on the field distribution of the symmetric mode of the whole structure, while antisymmetric will have none - and the other way around for antisymmetric modes. Therefore only even modes are used in Fig. 2.5.

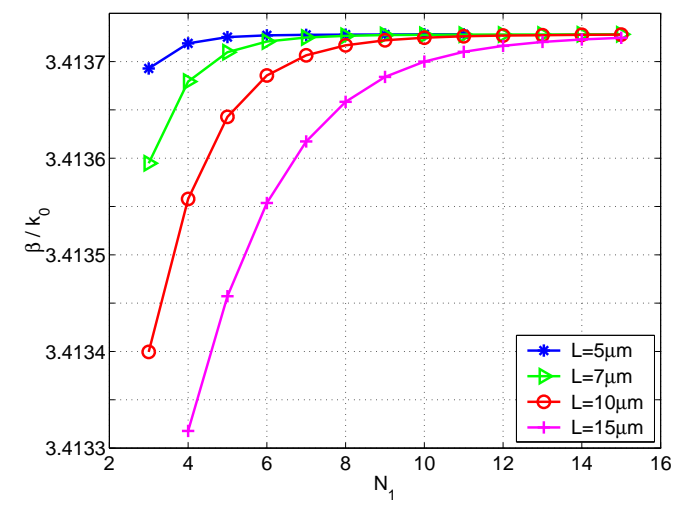

Figure 2.5: Convergence of the effective index $(\beta / k)$ of the fundamental TE mode for the rib waveguide of Fig. 2.2 $(b)(h=0.4 \mu \mathrm{m})$ as a function of the number of even modes from region $S_{3}$, used in the expansion (2.3.3), for different sizes of the computational window $x \in[-L, L]$. The number of even modes from the region $S_{4}$ (equivalently, $S_{1}$ or $S_{2}$ ), used in the expansion (2.3.3), is always 5.

Already with a moderate number of $15+5$ basis fields a satisfactory convergence in refractive index is achieved, contrary to the results of the Complex General Fourier Variational Method in [69] where many more basis modes are needed.

While according to the figure the effective index $\beta / k$ obtained on a larger computational window $L$ is always smaller than the effective index computed with a smaller window for the same number of (different) modes $N$, this is not the case when high number of terms are taken into account in expansion (2.3.3) (the curves cross). As was discussed in section 2.2, when using the full modal basis sets with increasing computational window the effective index (propagation constant) of the fundamental mode can only increase. 
As discussed in section 2.3.2, when taking only one mode into the expansion (VMEM[1,0]) the resulting equations are still always well-defined. Contrary to the effective index method, the VMEM produces reasonable results after the outer slice has gone below cut-off, at very similar computational cost.

\subsubsection{Four evanescently coupled ribs}

For the analysis of coupler structures, a basic issue is the accurate determination of all "supermode" propagation constants, i.e. fundamental as well as higher order modes are relevant. While finite difference and finite element methods can produce rather accurate results, they require large mesh sizes to cover the waveguide cross-section. The VMEM proves to be applicable for this type of structures.

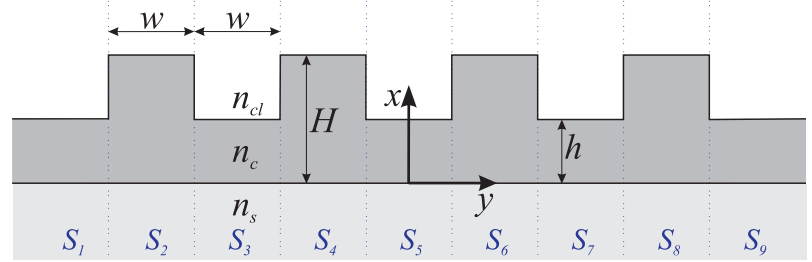

Figure 2.6: Geometrical parameters of the four rib waveguide structure: $w=4 \mu m, H=6 \mu m, h=3 \mu m$, refractive indices: $n_{s}=3.34, n_{c}=3.44$, $n_{c l}=1.0$, operating wavelength $\lambda=1.55 \mu \mathrm{m}$. A computational window $x \in[-10,10] \mu m$ has been used.

Fig. 2.6 introduces a waveguide geometry with four parallel ribs [67]. It can be divided into 9 slices $S_{1}, \ldots, S_{9}$ with horizontally homogeneous refractive index distributions. In the field expansion we will take into account modes from two slices, $S_{2}$ (alternatively, any other even numbered slice) and $S_{3}$ (alternatively, any other odd numbered slice). Table 1 compares normalized propagation constants with results from other methods. Note that even with only quite few modes $X_{i}$ in the expansion (2.3.3) reasonable accuracy in comparison with FEMLAB results can be achieved. The deviation in propagation constants from results of other methods can be due to polarization effects, i.e. due to different approximate mode equations. In Fig. 2.7 corresponding field profiles are shown. 


\begin{tabular}{c|c|c|c|c}
\hline$b$ & TE0 & TE1 & TE2 & TE3 \\
\hline DSI & 0.944760 & 0.943999 & 0.942957 & 0.942008 \\
EIM & 0.959920 & 0.959118 & 0.958025 & 0.957040 \\
FEMLAB & 0.946091 & 0.945366 & 0.944381 & 0.943493 \\
\hline VMEM[3,2] & 0.945826 & 0.945099 & 0.944104 & 0.943199 \\
VMEM $[30,2]$ & 0.946113 & 0.945392 & 0.944407 & 0.943513 \\
\hline \hline$b$ & TM0 & TM1 & TM2 & TM3 \\
\hline DSI & 0.944509 & 0.943852 & 0.942975 & 0.942195 \\
EIM & 0.958671 & 0.957919 & 0.956907 & 0.956006 \\
FEMLAB & 0.943087 & 0.942395 & 0.941462 & 0.940628 \\
\hline VMEM[3,2] & 0.942558 & 0.941863 & 0.940917 & 0.940062 \\
VMEM $[30,2]$ & 0.943019 & 0.942314 & 0.941381 & 0.940539 \\
\hline
\end{tabular}

Table 1. Normalized effective indices $b=\left((\beta / k)^{2}-n_{s}^{2}\right) /\left(n_{c}^{2}-n_{s}^{2}\right)$ of the four rib waveguide of Fig. 2.6. DSI: discrete-spectral-index method by $\mathrm{Ng}$ et. al. [67], EIM: effective index method, FEMLAB: finite element calculation on a dense mesh and a computational window $(x, y) \in[-10,10] \times$ $[-20,20] \mu m^{2}, V M E M[i, j]$ : the present method, where a computational window $x \in[-10,10] \mu m$ and $i$ and $j$ modes of the slices $S_{2}$ and $S_{3}$ correspondingly were used in the expansion (2.3.3).

\subsubsection{D coupler}

Fig. 2.8 shows the geometry of a three dimensional four waveguide coupler [24], [60]. This waveguide can be divided into five slices with homogeneous refractive index distribution along the $y$-direction. We performed two calculations: one on an infinite and one on a finite computational window. In the former case only two - one symmetric and one antisymmetric - guided modes exist in the slice $S_{2}$ (alternatively, $S_{4}$ ) and none in the slice $S_{3}$ (alternatively, $S_{1}$ or $S_{5}$ ). It is obvious, that only symmetric modes of constituting slices will influence the estimation of propagation constants and field profiles of symmetric, with respect to $y$-axis, modes of the whole waveguide, while antisymmetric modes will be irrelevant, and vice versa. If a computational window $x \in\left[x_{0}, x_{1}\right]$ is used, complete sets of modes exist in all five slices. The reasoning of choosing either symmetric or antisymmetric modes remains valid.

Table 2 compares effective indices, obtained by various other methods and the present one. To calculate propagation constants of modes TE00 and TE01 (TE10 and TE11) only symmetric (antisymmetric) modes were 

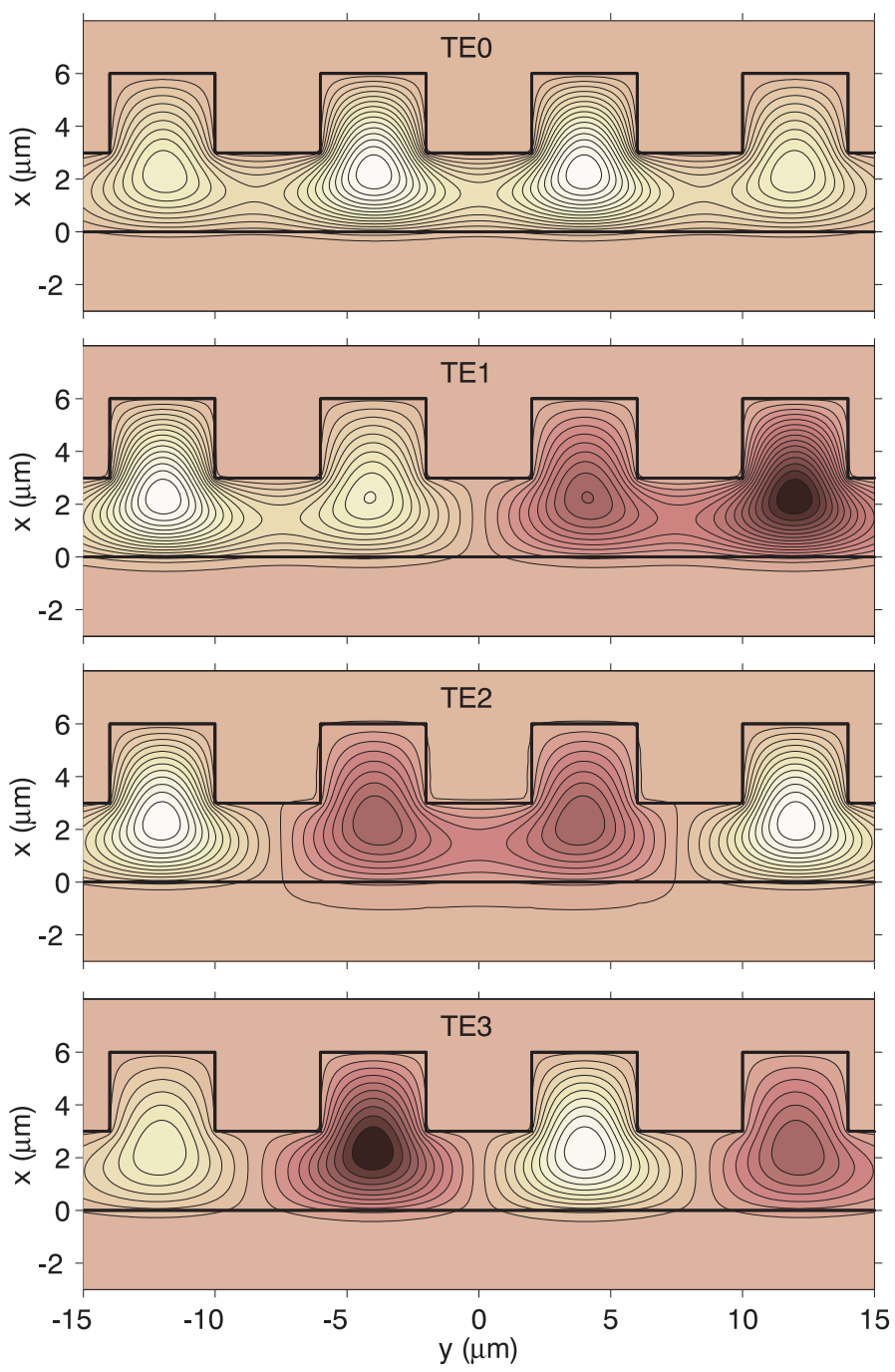

Figure 2.7: VMEM[30, 2] field profiles of the four rib waveguide of the Fig. 3 for the propagation constants of Table 1.

used. Rather accurate estimations of effective indices are achieved already with only one mode in the expansion. Fig. 2.9 shows the corresponding field profiles.

Due to the fact that slices $S_{1}, S_{3}, S_{5}$ do not support any guided modes effective indices of these slices are not defined and thus the "standard" EIM cannot be consistently applied to this waveguide geometry. As an 


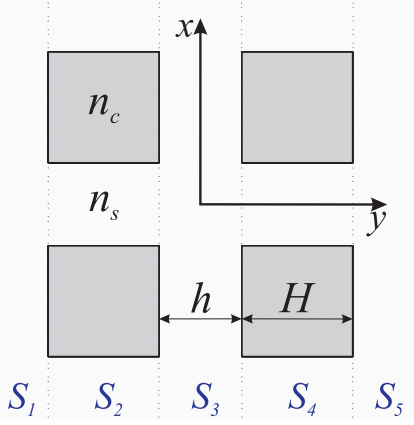

Figure 2.8: A 3D waveguide coupler - four equidistant square cores of higher refractive index embedded in a substrate of lower refractive index [24]. Parameters are $h=3 \mu m, H=4 \mu m, n_{s}=1.506, n_{c}=1.512$, operating wavelength $\lambda=1.32 \mu \mathrm{m}$.

approximation to these indices the substrate refractive index $n_{s}$ was used to compute the EIM effective indices of Table 2. Note that EIM profiles are always discontinuous across the interfaces, while the VMEM fields are well defined and continuous even with one mode in the expansion (2.3.3).

\begin{tabular}{c|cccc}
\hline & $N_{\text {eff }}^{00}$ & $N_{\text {eff }}^{01}$ & $N_{\text {eff }}^{10}$ & $N_{\text {eff }}^{11}$ \\
\hline VFEM & 1.5075807 & 1.5067966 & 1.5067966 & 1.5060260 \\
WMM & 1.5078966 & 1.5071085 & 1.5071092 & 1.5064697 \\
EIM & 1.5080433 & 1.5072134 & 1.5075570 & 1.5067277 \\
\hline VMEM $^{*}[1,0]$ & 1.5077912 & 1.5069894 & 1.5069690 & 1.5061836 \\
VMEM $[15,2]$ & 1.5078853 & 1.5070795 & 1.5070793 & 1.5062961 \\
\hline
\end{tabular}

Table 2. Effective indices $N_{\text {eff }}=\beta / k$ of the TE modes of the $3 D$ coupler; VFEM: vectorial FEM [24], WMM: wave matching method [60], EIM: effective index method, VMEM[i,j]: the present method with $i$ and $j$ modes of slices $S_{2}$ and $S_{3}$ correspondingly, used in the field expansion. For the results VMEM* an infinite computational window was used and for VMEM the computational window was $x \in[-20,20] \mu m$.

\subsection{Concluding remarks}

A variational method for mode analysis of dielectric waveguides was developed. VMEM results were compared with several other methods on differ- 
(a)

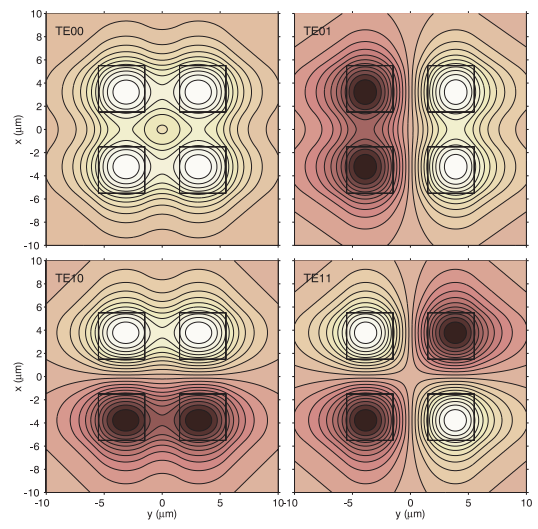

(b)

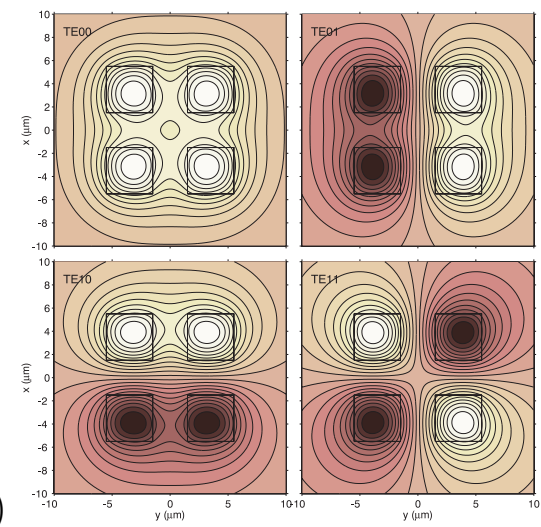

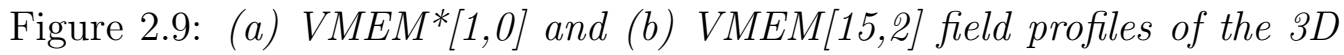
coupler.

ent waveguide geometries. Somewhat remarkably, the comparison indicates that a reasonable accuracy in the computation of propagation constants can be achieved with rather few (sometimes: single) modes in the field expansion. No problem arises, if one of the constituting slices is below cut-off.

It was shown that the VMEM is applicable to waveguides with arbitrary, piecewise constant rectangular cross-section. In principle, also waveguides with arbitrary refractive index distributions (graded index, non-rectangular discontinuities) can be considered, provided these can reasonably be approximated in a staircase manner by slices with piecewise constant rectangular refractive index distribution [107].

Although in this chapter we concentrated on scalar / semivectorial analysis, it will be shown that it is possible to extend this method to vectorial calculations by using the stationary formula for the vectorial mode equations [101] together with suitable templates for the vectorial fields.

\subsection{Appendix}

If TM polarized modes are considered, some of the equations in sections 2.2 and 2.3 have to be modified as follows. The principal magnetic field 
component $H=H_{y}(x, y)$ is to satisfy the scalar TM mode equation

$$
\nabla\left(\frac{1}{n^{2}(x, y)} \nabla H\right)+k^{2} H=\beta^{2} \frac{1}{n^{2}(x, y)} H .
$$

Propagation constants are found as values of the functional

$$
\mathcal{F}(H)=\frac{\int\left[-\frac{1}{n^{2}(x, y)}|\nabla H(x, y)|^{2}+k^{2} H^{2}(x, y)\right] d x d y}{\int \frac{1}{n^{2}(x, y)} H^{2}(x, y) d x d y}
$$

at critical points. When restricted to the field template (2.3.3) with TM modes, (A-2) reads

$$
\mathcal{F}\left(Y_{1}, \ldots, Y_{N}\right)=\frac{\int\left[-\frac{1}{n^{2}(x, y)}\left|\nabla\left(\sum_{i=1}^{N} X_{i}(x) Y_{i}(y)\right)\right|^{2}+k^{2}\left(\sum_{i=1}^{N} X_{i}(x) Y_{i}(y)\right)^{2}\right] d x d y}{\int \frac{1}{n^{2}(x, y)}\left(\sum_{i=1}^{N} X_{i}(x) Y_{i}(y)\right)^{2} d x d y} .
$$

Evaluation of the condition for stationarity leads to the equation

$$
\left(\mathbf{F}(y) \mathbf{Y}^{\prime}\right)^{\prime}+\mathbf{M}(y) \mathbf{Y}=\beta^{2} \mathbf{F}(y) \mathbf{Y}
$$

with $\mathbf{Y}$ and $\mathbf{F}(y) \mathbf{Y}^{\prime}$ required to be continuous across the slice interfaces, where the entries of the matrices $\mathbf{F}$ and $\mathbf{M}$ are

$$
\begin{gathered}
\mathbf{F}_{g, h}=\int_{x_{0}}^{x_{1}} \frac{1}{n^{2}(x, y)} X_{g}(x) X_{h}(x) d x, \\
\mathbf{M}_{g, h}=\int_{x_{0}}^{x_{1}}\left(k^{2} X_{g} X_{h}-\frac{1}{n^{2}(x, y)} X_{g}^{\prime} X_{h}^{\prime}\right) d x .
\end{gathered}
$$

The function $\mathbf{Y}$ in each separate slice satisfies the vectorial differential equation with constant coefficients

$$
\left(\mathbf{Y}^{(j)}\right)^{\prime \prime}+\mathbf{T}^{(j)} \mathbf{Y}^{(j)}=\beta^{2} \mathbf{Y}^{(j)}, \text { where } \mathbf{T}^{(j)}=\left(\mathbf{F}^{(j)}\right)^{-1} \mathbf{M}^{(j)},
$$

and the continuity conditions are (2.3.12) and

$$
\mathbf{F}^{(j)}\left(\mathbf{Y}^{(j)}\left(y^{(j)}\right)\right)^{\prime}=\mathbf{F}^{(j+1)}\left(\mathbf{Y}^{(j+1)}\left(y^{(j)}\right)\right)^{\prime}, \quad j=1, \ldots, r-1 .
$$


Using the exponential form $\exp \left(\mu^{(j)} y\right) \mathbf{p}_{j}$ for $\mathbf{Y}^{(j)}$ within the slice $S_{j}$ one obtains a system of equations in the form of (2.3.14), where the columns of the submatrices are given by

$$
\begin{aligned}
& \mathbf{V}^{d}(i)=\left[\begin{array}{c}
\exp \left(\mu_{i}^{(1)} y^{(1)}\right) \mathbf{p}_{i}^{(1)} \\
\mu_{i}^{(1)} \exp \left(\mu_{i}^{(1)} y^{(1)}\right) \mathbf{F}^{(1)} \mathbf{p}_{i}^{(1)}
\end{array}\right], \quad \mathbf{V}^{c}(i)=\left[\begin{array}{c}
-\exp \left(-\mu_{i}^{(r)} y^{(r-1)}\right) \mathbf{p}_{i}^{(r)} \\
\mu_{i}^{(r)} \exp \left(-\mu_{i}^{(r)} y^{(r-1)}\right) \mathbf{F}^{(r)} \mathbf{p}_{i}^{(r)}
\end{array}\right], \\
& \mathbf{A}_{j-}(i)=\left[\begin{array}{c}
-\exp \left(\mu_{i}^{(j)} y^{(j-1)}\right) \mathbf{p}_{i}^{(j)} \\
-\mu_{i}^{(j)} \exp \left(\mu_{i}^{(j)} y^{(j-1)}\right) \mathbf{F}^{(j)} \mathbf{p}_{i}^{(j)}
\end{array}\right], \quad \mathbf{B}_{j-}(i)=\left[\begin{array}{c}
-\exp \left(-\mu_{i}^{(j)} y^{(j-1)}\right) \mathbf{p}_{i}^{(j)} \\
\mu_{i}^{(j)} \exp \left(-\mu_{i}^{(j)} y^{(j-1)}\right) \mathbf{F}^{(j)} \mathbf{p}_{i}^{(j)}
\end{array}\right], \\
& \mathbf{A}_{j+}(i)=\left[\begin{array}{c}
\exp -1(6)\left(\mu_{i}^{(j)} y^{(j)}\right) \mathbf{p}_{i}^{(j)} \\
\mu_{i}^{(j)} \exp \left(\mu_{i}^{(j)} y^{(j)}\right) \mathbf{F}^{(j)} \mathbf{p}_{i}^{(j)}
\end{array}\right], \quad \mathbf{B}_{j+}(i)=\left[\begin{array}{c}
\exp \left(-\mu_{i}^{(j)} y^{(j)}\right) \mathbf{p}_{i}^{(j)} \\
-\mu_{i}^{(j)} \exp \left(-\mu_{i}^{(j)} y^{(j)}\right) \mathbf{F}^{(j)} \mathbf{p}_{i}^{(j)}
\end{array}\right] .
\end{aligned}
$$




\section{Vectorial mode problems}

A flexible and efficient method for fully vectorial modal analysis of 3D dielectric optical waveguides with arbitrary $2 \mathrm{D}$ cross-sections is proposed. The technique is based on expansion of each modal component in some a priori defined functions defined on one coordinate axis times some unknown coefficient-functions, defined on the other axis. By applying a variational restriction procedure the unknown coefficientfunctions are determined, resulting in an optimum approximation of the true vectorial mode profile. This technique can be related to both Effective Index and Mode Matching methods. A couple of examples illustrate the performance of the method.

\subsection{Introduction}

Three-dimensional optical channel waveguides are basic components of integrated optical devices such as directional couplers, wavelength filters, phase shifters, and optical switches. The successful design of these devices requires an accurate estimation of the modal field profiles and propagation constants. Over already some decades several classes of methods for the analysis of dielectric optical waveguides were developed: among these are techniques of more numerical character, like Finite Element and Finite Difference approximations, the Method of Lines, and Integral Equations Methods, but also more analytical approaches like Film Mode Matching (FMM) and the Effective Index Method (EIM). Detailed overviews of these techniques can be found in [16], [102], [82]. 
In the present chapter we propose an extension of the scalar mode solver of Chapter 2 to vectorial problems. Our method is based on individual expansions of each mode profile component into a set of a priori defined functions of one coordinate axis (vertical), here, field components of some slab waveguide mode. The expansion is global, meaning that the same basis functions are used at any point on the horizontal axis. The unknown expansion coefficients - in our case functions, defined on the horizontal axis - are found by means of variational methods [101], [31].

The present method can be viewed as some bridge between two popular approaches, namely the FMM on the one hand and the EIM on the other. In the standard EIM the 2D problem of finding modes of the waveguide is reduced to consecutive solving two 1D problems: at first, the 1D modes, and their propagation constants, of the constituting slab waveguides are found, and then their propagation constants are used to define effective refractive indices of a reduced 1D problem. In general this is a very quick and easy approach for a rough estimation of mode parameters. However, in case one of the constituting slabs doesn't support a guided mode (for example, some substrate material with air on top) it is impossible to uniquely define the effective refractive index in that particular region of the reduced problem. Should it be the refractive index of the cladding, refractive index of the air, or something in between? The restriction of the present approach to one-term expansions will answer this question.

The validity of the method was checked on several structures, including waveguides with rectangular and non-rectangular piecewise-constant refractive index distributions, and a diffused waveguide. Comparison shows that the present method is a more consistent and accurate alternative to the standard EIM and also can be pushed to its limits and used for more rigorous computations.

The chapter is organized as follows. In section 3.2 the problem of finding vectorial modes of the dielectric waveguides is stated, then some properties of slab modes and the modal field ansatz are described in sections 3.3 and 3.4. The equations for the coefficient functions are derived in section 3.5. Section 3.6 outlines the numerical solution methods. The relation of the present method to the EIM and FMM is explained in more detail in sections 3.7 and 3.8. Then in section 3.9 numerical results for several waveguide configurations are presented. Finally some concluding remarks are made in section 3.10 . 


\subsection{Variational form of the vectorial mode problem}

Consider a $z$-invariant dielectric isotropic waveguide defined on its crosssection by a refractive index $n(x, y)$ or relative dielectric permittivity distribution $\varepsilon(x, y)=n^{2}(x, y)$. Figure 3.1 shows two examples.
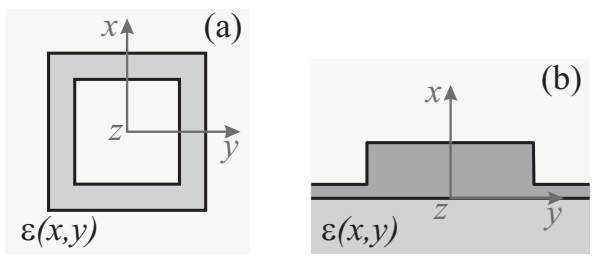

Figure 3.1: Examples for 3D dielectric waveguides defined on their cross-section by permittivity distribution $\varepsilon(x, y)$. The structures are invariant along the z-axis. (a) boxshaped hollow-core waveguide, a concept from [66], the subject of section 3.9.1, (b) a standard rib waveguide, investigated in section 3.9.2.

The propagation of monochromatic light, given by the electric $\overline{\mathbf{E}}$ and magnetic $\overline{\mathbf{H}}$ components of the optical field, with propagation constant $\beta$ and frequency $\omega$,

$$
\overline{\mathbf{E}}(x, y, z, t)=\mathbf{E}(x, y) \mathrm{e}^{-\mathrm{i} \beta z} \mathrm{e}^{\mathrm{i} \omega t}, \quad \overline{\mathbf{H}}(x, y, z, t)=\mathbf{H}(x, y) \mathrm{e}^{-\mathrm{i} \beta z} \mathrm{e}^{\mathrm{i} \omega t},
$$

is governed by the Maxwell's equations for the mode profile components $\mathbf{E}$ and $\mathbf{H}$

$$
\begin{aligned}
& \omega \varepsilon_{0} \varepsilon \mathbf{E}+\mathrm{i} C \mathbf{H}=\beta R \mathbf{H}, \\
& \omega \mu_{0} \mu \mathbf{H}-\mathrm{i} C \mathbf{E}=-\beta R \mathbf{E},
\end{aligned}
$$

with

$$
R=\left(\begin{array}{ccc}
0 & 1 & 0 \\
-1 & 0 & 0 \\
0 & 0 & 0
\end{array}\right), \quad C=\left(\begin{array}{ccc}
0 & 0 & \partial_{y} \\
0 & 0 & -\partial_{x} \\
-\partial_{y} & \partial_{x} & 0
\end{array}\right)
$$

vacuum permittivity $\varepsilon_{0}$, vacuum permeability $\mu_{0}$, relative permittivity $\varepsilon(x, y)=n^{2}(x, y)$. Here and further in this chapter it is assumed that the relative permeability $\mu$ is equal to 1 , as is the case for most materials at optical frequencies.

We will work with a variational formulation of the Maxwell's equations. Solutions $(\beta, \mathbf{E}, \mathbf{H})$ of the equations (3.2.2) correspond to stationary points 
$(\mathbf{E}, \mathbf{H})$ of the functional [101]

$$
\mathcal{F}(\mathbf{E}, \mathbf{H})=\frac{\omega \varepsilon_{0}\langle\mathbf{E}, \varepsilon \mathbf{E}\rangle+\omega \mu_{0}\langle\mathbf{H}, \mathbf{H}\rangle+\mathrm{i}\langle\mathbf{E}, C \mathbf{H}\rangle-\mathrm{i}\langle\mathbf{H}, C \mathbf{E}\rangle}{\langle\mathbf{E}, R \mathbf{H}\rangle-\langle\mathbf{H}, R \mathbf{E}\rangle},
$$

with propagation constant $\beta=\mathcal{F}(\mathbf{E}, \mathbf{H})$ equal to the value of the functional at the stationary point. The inner product used is $\langle\mathbf{A}, \mathbf{B}\rangle=\int \mathbf{A}^{*} \cdot \mathbf{B} d x d y$. The natural interface conditions are the continuity of all tangential field components across the interfaces.

\subsection{Slab modes}

In this section we will consider modes of slab waveguides, which we will use in the next section as building blocks to construct approximations of the modes of waveguides with arbitrary $2 \mathrm{D}$ cross-sections.

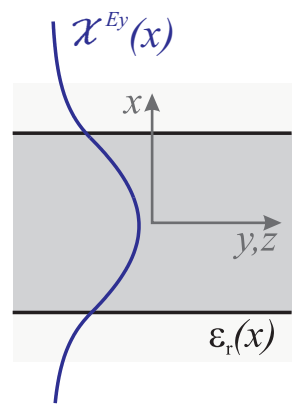

Figure 3.2: A slab waveguide with permittivity distribution $\varepsilon_{r}(x)$ and principal component $\chi^{E_{y}}$ of a corresponding TE slab mode (3.3.5).

A one dimensional TE mode, propagating in the $z$-direction with propagation constant $\beta_{\mathrm{r}, \mathrm{TE}}$, of the slab waveguide, given by the permittivity distribution $\varepsilon_{\mathrm{r}}(x)$ (Figure 3.2) can be represented as

$$
\left(\begin{array}{c}
E_{x}, E_{y}, E_{z} \\
H_{x}, H_{y}, H_{z}
\end{array}\right)(x, y, z)=\left(\begin{array}{ccc}
0, & \chi^{E_{y}}(x), & 0 \\
\chi^{H_{x}}(x), & 0, & \chi^{H_{z}}(x)
\end{array}\right) \mathrm{e}^{-\mathrm{i} \beta_{\mathrm{r}, \mathrm{TE}} z} .
$$

The principal electric component $\chi^{E_{y}}$ satisfies the equation

$$
\left(\chi^{E_{y}}(x)\right)^{\prime \prime}+k^{2} \varepsilon_{\mathrm{r}}(x) \chi^{E_{y}}(x)=\beta_{\mathrm{r}, \mathrm{TE}}^{2} \chi^{E_{y}}(x)
$$

with vacuum wavenumber $k=2 \pi / \lambda$. The remaining two nonzero components of the mode profile can be derived directly from $\chi^{E_{y}}$ :

$$
\chi^{H_{x}}(x)=-\frac{\beta}{\omega \mu_{0}} \chi^{E_{y}}(x), \quad \chi^{H_{z}}(x)=\frac{\mathrm{i}}{\omega \mu_{0}}\left(\chi^{E_{y}}(x)\right)^{\prime} .
$$


The slab waveguide (Figure 3.2) is by definition invariant in the $(y, z)$-plane. So if a modal solution of Maxwell's equations propagating in the $z$-direction will be rotated in the $(y, z)$-plane by an angle $\theta$ (Figure 3.3), it will still remain a modal solution of the Maxwell's equations, but now propagating in the direction $(y, z)=(-\sin \theta ; \cos \theta)$ :

$$
\begin{array}{r}
\left(\begin{array}{c}
E_{x}, E_{y}, E_{z} \\
H_{x}, H_{y}, H_{z}
\end{array}\right)(x, y, z)=\left(\begin{array}{ccc}
0, & \chi^{E_{y}}(x) \cos \theta, & \chi^{E_{y}}(x) \sin \theta \\
\chi^{H_{x}}(x), & -\chi^{H_{z}}(x) \sin \theta, & \chi^{H_{z}}(x) \cos \theta
\end{array}\right) . \\
\cdot \mathrm{e}^{-\mathrm{i} \beta_{\mathrm{r}, \mathrm{TE}}(-\sin \theta y+\cos \theta z)} .
\end{array}
$$

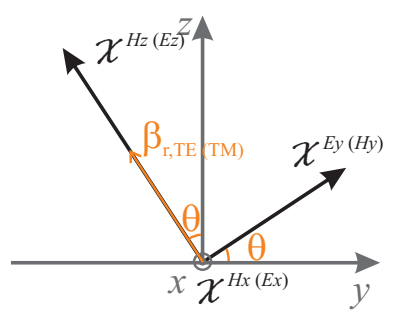

Figure 3.3: A slab TE (TM) mode propagating in the z-direction with propagation constant $\beta_{r, T E(T M)}$ is rotated around the $x$-axis by an angle $\theta$. The rotated mode propagates with the same propagation constant, but in the direction $(y, z)=(-\sin \theta, \cos \theta)$.

Similarly a 1D TM slab mode, propagating in the $z$-direction with propagation constant $\beta_{\mathrm{r}, \mathrm{TM}}$

$$
\left(\begin{array}{c}
E_{x}, E_{y}, E_{z} \\
H_{x}, H_{y}, H_{z}
\end{array}\right)(x, y, z)=\left(\begin{array}{ccc}
\chi^{E_{x}}(x), & 0, & \chi^{E_{z}}(x) \\
0, & \chi^{H_{y}}(x), & 0
\end{array}\right) \mathrm{e}^{-\mathrm{i} \beta_{\mathrm{r}, \mathrm{TM}} z}
$$

will still be a solution of the Maxwell's equations after a rotation around the $x$-axis (Figure 3.3)

$$
\begin{array}{r}
\left(\begin{array}{c}
E_{x}, E_{y}, E_{z} \\
H_{x}, H_{y}, H_{z}
\end{array}\right)(x, y, z)=\left(\begin{array}{ccc}
\chi^{E_{x}}(x), & -\chi^{E_{z}}(x) \sin \theta, & \chi^{E_{z}}(x) \cos \theta \\
0, & \chi^{H_{y}}(x) \cos \theta, & \chi^{H_{y}}(x) \sin \theta
\end{array}\right) \\
\cdot \mathrm{e}^{-\mathrm{i} \beta_{\mathrm{r}, \mathrm{TM}}(-\sin \theta y+\cos \theta z)}
\end{array}
$$

The principal magnetic component $\chi^{H_{y}}$ satisfies the equation

$$
\left(\frac{1}{\varepsilon_{\mathrm{r}}(x)}\left(\chi^{H_{y}}(x)\right)^{\prime}\right)^{\prime}+k^{2} \chi^{H_{y}}(x)=\beta_{\mathrm{r}, \mathrm{TM}}^{2} \frac{1}{\varepsilon_{\mathrm{r}}(x)} \chi^{H_{y}}(x) .
$$

Again the remaining two nonzero components of the mode profile can be derived directly from $\chi^{H_{y}}$ :

$$
\chi^{E_{x}}(x)=\frac{\beta}{\omega \varepsilon_{0} \varepsilon_{\mathrm{r}}(x)} \chi^{H_{y}}(x), \quad \chi^{E_{z}}(x)=-\frac{\mathrm{i}}{\omega \varepsilon_{0} \varepsilon_{\mathrm{r}}(x)}\left(\chi^{H_{y}}(x)\right)^{\prime} .
$$




\subsection{Modal field ansatz}

We now return to the vectorial modes of the $3 \mathrm{D}$ waveguides, as in section 3.2. Each field component $F \in\left\{E_{x}, E_{y}, E_{z}, H_{x}, H_{y}, H_{z}\right\}$ is represented individually as a superposition of $m_{F}$ a priori known functions $X_{j}^{F}(x)$, defined on one coordinate axis, times some unknown coefficient-function $Y_{j}^{F}(y)$, defined on the other axis:

$$
F(x, y)=\sum_{j=1}^{m_{F}} X_{j}^{F}(x) Y_{j}^{F}(y) .
$$

For the functions $X$ we will take components of slab modes from some reference slice(s). Further in the chapter two types of the expansion will be relevant, one which introduces 5 unknown functions $Y$ per slab mode, and another one, which introduces only 3 . These will be called five component approximation (VEIM5) and three component approximation (VEIM3), respectively.

In case of VEIM5, the TE basis mode (3.3.5) number $j$ with mode profile components $\chi_{j}^{E_{y}}, \chi_{j}^{H_{x}}, \chi_{j}^{H_{z}}$ contributes to the expansion of components $E_{y}$, $E_{z}, H_{x}, H_{y}$ and $H_{z}$ with the form $\chi_{j}^{E_{y}} Y_{j}^{E_{y}}, \chi_{j}^{E_{y}} Y_{j}^{E_{z}}, \chi_{j}^{H_{x}} Y_{j}^{H_{x}}, \chi_{j}^{H_{z}} Y_{j}^{H_{y}}$ and $\chi_{j}^{H_{z}} Y_{j}^{H_{z}}$. Likewise, the TM basis mode (3.3.9) number $l$ with mode profile components $\chi_{l}^{E_{y}}, \chi_{l}^{H_{x}}, \chi_{l}^{H_{z}}$ contributes to the expansion of components $E_{x}, E_{y}, E_{z}, H_{y}$ and $H_{z}$ with the form $\chi_{l}^{E_{x}} Y_{l}^{E_{x}}, \chi_{l}^{E_{z}} Y_{l}^{E_{y}}, \chi_{l}^{E_{z}} Y_{l}^{E_{z}}, \chi_{l}^{H_{y}} Y_{l}^{H_{y}}$, $\chi_{l}^{H_{y}} Y_{l}^{H_{z}}$, such that the complete expansion looks like

$$
\begin{aligned}
& \left(\begin{array}{c}
E_{x}, E_{y}, E_{z} \\
H_{x}, H_{y}, H_{z}
\end{array}\right)(x, y, z)= \\
& \quad=\sum_{j \in \mathrm{TE}}\left(\begin{array}{ccc}
0, & \chi_{j}^{E_{y}}(x) Y_{j}^{E_{y}}(y), & \chi_{j}^{E_{y}}(x) Y_{j}^{E_{z}}(y) \\
\chi_{j}^{H_{x}}(x) Y_{j}^{H_{x}}(y), & \chi_{j}^{H_{z}}(x) Y_{j}^{H_{y}}(y), & \chi_{j}^{H_{z}}(x) Y_{j}^{H_{z}}(y)
\end{array}\right)+ \\
& \quad+\sum_{l \in \mathrm{TM}}\left(\begin{array}{ccc}
\chi_{l}^{E_{x}}(x) Y_{l}^{E_{x}}(y), & \chi_{l}^{E_{z}}(x) Y_{l}^{E_{y}}(y), & \chi_{l}^{E_{z}}(x) Y_{l}^{E_{z}}(y) \\
0, & \chi_{l}^{H_{y}}(x) Y_{l}^{H_{y}}(y), & \chi_{l}^{H_{y}}(x) Y_{l}^{H_{z}}(y)
\end{array}\right) .
\end{aligned}
$$

This expansion has the drawback that the functions making up some of the components can become linearly dependent. In case of VEIM3 we omit contributions of some modal components. So a TE basis mode (3.3.5) number $j$ with mode profile components $\chi_{j}^{E_{y}}, \chi_{j}^{H_{x}}, \chi_{j}^{H_{z}}$ contributes to the expansion of components $E_{y}, E_{z}$ and $H_{x}$ with the form $\chi_{j}^{E_{y}} Y_{j}^{E_{y}}, \chi_{j}^{E_{y}} Y_{j}^{E_{z}}$, 
$\chi_{j}^{H_{x}} Y_{j}^{H_{x}}$. Likewise a TM basis mode (3.3.9) number $l$ with mode profile components $\chi_{l}^{E_{y}}, \chi_{l}^{H_{x}}, \chi_{l}^{H_{z}}$ contributes to the expansion of components $E_{x}$, $H_{y}$ and $H_{z}$ with the form $\chi_{l}^{E_{x}} Y_{l}^{E_{x}}, \chi_{l}^{H_{y}} Y_{l}^{H_{y}}, \chi_{l}^{H_{y}} Y_{l}^{H_{z}}$, such that the complete expansion looks like

$$
\begin{aligned}
& \left(\begin{array}{c}
E_{x}, E_{y}, E_{z} \\
H_{x}, H_{y}, H_{z}
\end{array}\right)(x, y, z)= \\
& \quad=\sum_{j \in \mathrm{TE}}\left(\begin{array}{ccc}
0, & \chi_{j}^{E_{y}}(x) Y_{j}^{E_{y}}(y), & \chi_{j}^{E_{y}}(x) Y_{j}^{E_{z}}(y) \\
\chi_{j}^{H_{x}}(x) Y_{j}^{H_{x}}(y), & 0, & 0
\end{array}\right)+ \\
& \quad+\sum_{l \in \mathrm{TM}}\left(\begin{array}{ccc}
\chi_{l}^{E_{x}}(x) Y_{l}^{E_{x}}(y), & 0, & 0 \\
0, & \chi_{l}^{H_{y}}(x) Y_{l}^{H_{y}}(y), & \chi_{l}^{H_{y}}(x) Y_{l}^{H_{z}}(y)
\end{array}\right) .
\end{aligned}
$$

Note that in both expansions each contributing component $\chi^{F}$ of $1 \mathrm{D}$ mode is used to represent the field not only in the slab segment where it belongs to, as in EIM and FMM methods, but also in the whole waveguide. So even with a single slab mode in both expansions, (3.4.14) and (3.4.15), it is possible to construct an approximation of the field in the whole structure. In section 3.7 we will study in detail properties of such one-mode-expansions.

The form of the expansion (3.4.14) was inspired by the mode matching techniques that use rotated modes (3.3.8), (3.3.10) to locally expand the total field [5], [95]. In the present approach though, we attribute those parts of the slab mode components that do not depend on $x$ to the functions $Y^{F}$, treating them as unknowns. In the sections 3.7 and 3.8 we will study the behavior of these functions $Y^{F}$.

What concerns the choice of the reference slice(s), it seems that modal components from the slice, where the maximum power is expected to be localized, give the best results. Further in this chapter VEIM5 will be used with a few modes only for rough and efficient approximations, while VEIM3 will be used with higher numbers of modes to obtain accurate, converged results.

In the following all the slab mode components $\chi$, which are used to expand a field component $F$ of the complete waveguide, we will denote as $X^{F}$ (just like in eqn. (3.4.13)). 


\subsection{Reduced problem}

The next question is how to find corresponding functions $Y$, such that the expansion (3.4.13) represents the true solution in the best possible way. For this purpose we apply variational restriction [102], [31] of the functional (3.2.4). In short it can be outlined as follows. As it was already mentioned the critical points of the functional (3.2.4), which satisfy some continuity conditions, are solutions of the Maxwell's equations (3.2.2) and, vice versa, solutions of the Maxwell's equations (3.2.2) are critical points of the functional (3.2.4).

After insertion of the expansions (3.4.14) or (3.4.15), variation of the functional (3.2.4) with respect to a function $\mathbf{Y}^{F}$, a vector function made up of all functions $Y^{F}$, results in the following system of first order differential equations for $\mathbf{Y}^{F}$ with parameter $\beta$ :

$$
\begin{aligned}
& \mathbf{A}_{11} \mathbf{Y}^{E_{x}}+\mathbf{A}_{12}\left(\mathbf{Y}^{H_{z}}\right)^{\prime}=\beta \mathbf{A}_{13} \mathbf{Y}^{H_{y}} \\
& \mathbf{A}_{21} \mathbf{Y}^{E_{y}}+\mathbf{A}_{22} \mathbf{Y}^{H_{z}}=\beta \mathbf{A}_{23} \mathbf{Y}^{H_{x}} \\
& \mathbf{A}_{31} \mathbf{Y}^{E_{z}}+\mathbf{A}_{32}\left(\mathbf{Y}^{H_{x}}\right)^{\prime}+\mathbf{A}_{33} \mathbf{Y}^{H_{y}}=0 \\
& \mathbf{A}_{41} \mathbf{Y}^{H_{x}}+\mathbf{A}_{42}\left(\mathbf{Y}^{E_{z}}\right)^{\prime}=\beta \mathbf{A}_{43} \mathbf{Y}^{E_{y}} \\
& \mathbf{A}_{51} \mathbf{Y}^{H_{y}}+\mathbf{A}_{52} \mathbf{Y}^{E_{z}}=\beta \mathbf{A}_{53} \mathbf{Y}^{E_{x}} \\
& \mathbf{A}_{61} \mathbf{Y}^{H_{z}}+\mathbf{A}_{62}\left(\mathbf{Y}^{E_{x}}\right)^{\prime}+\mathbf{A}_{63} \mathbf{Y}^{E_{y}}=0 .
\end{aligned}
$$

The elements of the matrices $\mathbf{A}$ include the overlap integrals of the functions $X_{j}^{F}(x)$, their derivatives, and the local permittivity distribution of the waveguide:

$$
\begin{array}{lll}
\mathbf{A}_{11}(p, j)=\omega\left\langle X_{p}^{E_{x}}, \varepsilon X_{j}^{E_{x}}\right\rangle & \mathbf{A}_{12}(p, j)=\mathrm{i}\left\langle X_{p}^{E_{x}}, X_{j}^{H_{z}}\right\rangle & \mathbf{A}_{13}(p, j)=\left\langle X_{p}^{E_{x}}, X_{j}^{H_{y}}\right\rangle \\
\mathbf{A}_{21}(p, j)=\omega\left\langle X_{p}^{E_{y}}, \varepsilon X_{j}^{E_{y}}\right\rangle & \mathbf{A}_{22}(p, j)=-\mathrm{i}\left\langle X_{p}^{E_{y}},\left(X_{j}^{H_{z}}\right)^{\prime}\right\rangle & \mathbf{A}_{23}(p, j)=-\left\langle X_{p}^{E_{y}}, X_{j}^{H_{x}}\right\rangle \\
\mathbf{A}_{31}(p, j)=\omega\left\langle X_{p}^{E_{z}}, \varepsilon X_{j}^{E_{z}}\right\rangle & \mathbf{A}_{32}(p, j)=-\mathrm{i}\left\langle X_{p}^{E_{z}}, X_{j}^{H_{x}}\right\rangle & \mathbf{A}_{33}(p, j)=\mathrm{i}\left\langle X_{p}^{E_{z}},\left(X_{j}^{H_{y}}\right)^{\prime}\right\rangle \\
\mathbf{A}_{41}(p, j)=\omega \mu\left\langle X_{p}^{H_{x}}, X_{j}^{H_{x}}\right\rangle & \mathbf{A}_{42}(p, j)=-\mathrm{i}\left\langle X_{p}^{H_{x}}, X_{j}^{E_{z}}\right\rangle & \mathbf{A}_{43}(p, j)=-\left\langle X_{p}^{H_{x}}, X_{j}^{E_{y}}\right\rangle \\
\mathbf{A}_{51}(p, j)=\omega \mu\left\langle X_{p}^{H_{y}}, X_{j}^{H_{y}}\right\rangle & \mathbf{A}_{52}(p, j)=\mathrm{i}\left\langle X_{p}^{H_{y}},\left(X_{j}^{E_{z}}\right)^{\prime}\right\rangle & \mathbf{A}_{53}(p, j)=\left\langle X_{p}^{H_{y}}, X_{j}^{E_{x}}\right\rangle \\
\mathbf{A}_{61}(p, j)=\omega \mu\left\langle X_{p}^{H_{z}}, X_{j}^{H_{z}}\right\rangle & \mathbf{A}_{62}(p, j)=\mathrm{i}\left\langle X_{p}^{H_{z}}, X_{j}^{E_{x}}\right\rangle & \mathbf{A}_{63}(p, j)=-\mathrm{i}\left\langle X_{p}^{H_{z}},\left(X_{j}^{E_{y}}\right)^{\prime}\right\rangle
\end{array}
$$

Note that the permittivity appears only in $\mathbf{A}_{11}, \mathbf{A}_{21}$ and $\mathbf{A}_{31}$, hence only these matrices are $y$-dependent.

If the permittivity exhibits discontinuities along the $y$-direction, the functions

$$
\mathbf{Y}^{E_{x}} \text { and } \mathbf{Y}^{H_{x}}, \quad \mathbf{Y}^{E_{z}} \text { and } \mathbf{Y}^{H_{z}}
$$

are required to be continuous at the respective positions.

It turns out that by algebraic operations the system of first order differential equations (3.5.16) can be reduced to a system of second order 
differential equations for functions $\mathbf{Y}^{E_{x}}$ and $\mathbf{Y}^{H_{x}}$ only. Moreover, since the components $E_{y}$ and $E_{z}, H_{y}$ and $H_{z}$ are approximated by the same functions $\chi$ in the representations (3.4.14) and (3.4.15), the matrices $\mathbf{A}$ satisfy the following equalities:

$$
\begin{aligned}
& \mathbf{A}_{13}=-\mathrm{i} \mathbf{A}_{12} \\
& \mathbf{A}_{31}=\mathbf{A}_{21}, \quad \mathbf{A}_{32}=\mathrm{i} \mathbf{A}_{23}, \quad \mathbf{A}_{33}=-\mathbf{A}_{22} \\
& \mathbf{A}_{43}=-\mathrm{i} \mathbf{A}_{42} \\
& \mathbf{A}_{61}=\mathbf{A}_{51}, \quad \mathbf{A}_{62}=\mathrm{i} \mathbf{A}_{53}, \quad \mathbf{A}_{63}=-\mathbf{A}_{52},
\end{aligned}
$$

and hence the system (3.5.16) reduces to

$$
\mathbf{S}_{1} \mathbf{u}+\left(\mathbf{S}_{2} \mathbf{u}^{\prime}+\beta \mathbf{S}_{3} \mathbf{u}\right)^{\prime}=\beta^{2} \mathbf{S}_{2} \mathbf{u}+\beta \mathbf{S}_{3} \mathbf{u}^{\prime}
$$

with $\mathbf{u}(y)=\left(\begin{array}{c}\mathbf{Y}^{E_{x}}(y) \\ \mathbf{Y}^{H_{x}}(y)\end{array}\right)$ and (anti-)block-diagonal matrices $\mathbf{S}$ of the following form:

$$
\begin{aligned}
& \mathbf{S}_{1}=\left(\begin{array}{cc}
\mathbf{A}_{11} & 0 \\
0 & \mathbf{A}_{41}
\end{array}\right), \\
& \mathbf{S}_{2}=\left(\begin{array}{cc}
-\mathrm{i} \mathbf{A}_{12}\left(\mathbf{A}_{51}+\mathbf{A}_{52} \mathbf{A}_{21}^{-1} \mathbf{A}_{22}\right)^{-1} \mathbf{A}_{53} & 0 \\
0 & -\mathrm{i} \mathbf{A}_{42}\left(\mathbf{A}_{21}+\mathbf{A}_{22} \mathbf{A}_{51}^{-1} \mathbf{A}_{52}\right)^{-1} \mathbf{A}_{23}
\end{array}\right), \\
& \mathbf{S}_{3}=\left(\begin{array}{cc}
0 & \mathbf{A}_{12} \mathbf{A}_{51}^{-1} \mathbf{A}_{52}\left(\mathbf{A}_{21}+\mathbf{A}_{22} \mathbf{A}_{51}^{-1} \mathbf{A}_{52}\right)^{-1} \mathbf{A}_{23} \\
\mathbf{A}_{42} \mathbf{A}_{21}^{-1} \mathbf{A}_{22}\left(\mathbf{A}_{51}+\mathbf{A}_{52} \mathbf{A}_{21}^{-1} \mathbf{A}_{22}\right)^{-1} \mathbf{A}_{53} & 0
\end{array}\right) .
\end{aligned}
$$

Across the vertical interfaces continuity of

$$
\mathbf{u} \text { and } \mathbf{S}_{2} \mathbf{u}^{\prime}+\beta \mathbf{S}_{3} \mathbf{u}
$$

is required.

As soon as the function $\mathbf{u}$, or in other words $\mathbf{Y}^{E_{x}}$ and $\mathbf{Y}^{H_{x}}$, are known, the functions $\mathbf{Y}$ corresponding to the four other components can be derived as follows:

$$
\begin{aligned}
\mathbf{Y}^{E_{y}} & =\mathrm{i} \mathbf{A}_{21}^{-1} \mathbf{A}_{22}\left(\mathbf{A}_{51}+\mathbf{A}_{52} \mathbf{A}_{21}^{-1} \mathbf{A}_{22}\right)^{-1} \mathbf{A}_{53}\left(\mathbf{Y}^{E_{x}}\right)^{\prime}+\beta\left(\mathbf{A}_{21}+\mathbf{A}_{22} \mathbf{A}_{51}^{-1} \mathbf{A}_{52}\right)^{-1} \mathbf{A}_{23} \mathbf{Y}^{H_{x}}, \\
\mathbf{Y}^{E_{z}} & =\beta \mathbf{A}_{21}^{-1} \mathbf{A}_{22}\left(\mathbf{A}_{51}+\mathbf{A}_{52} \mathbf{A}_{21}^{-1} \mathbf{A}_{22}\right)^{-1} \mathbf{A}_{53} \mathbf{Y}^{E_{x}}-\mathrm{i}\left(\mathbf{A}_{21}+\mathbf{A}_{22} \mathbf{A}_{51}^{-1} \mathbf{A}_{52}\right)^{-1} \mathbf{A}_{23}\left(\mathbf{Y}^{H_{x}}\right)^{\prime}, \\
\mathbf{Y}^{H_{y}} & =\beta\left(\mathbf{A}_{51}+\mathbf{A}_{52} \mathbf{A}_{21}^{-1} \mathbf{A}_{22}\right)^{-1} \mathbf{A}_{53} \mathbf{Y}^{E_{x}}+\mathrm{i} \mathbf{A}_{51}^{-1} \mathbf{A}_{52}\left(\mathbf{A}_{21}+\mathbf{A}_{22} \mathbf{A}_{51}^{-1} \mathbf{A}_{52}\right)^{-1} \mathbf{A}_{23}\left(\mathbf{Y}^{H_{x}}\right)^{\prime}, \\
\mathbf{Y}^{H_{z}} & =-\mathrm{i}\left(\mathbf{A}_{51}+\mathbf{A}_{52} \mathbf{A}_{21}^{-1} \mathbf{A}_{22}\right)^{-1} \mathbf{A}_{53}\left(\mathbf{Y}^{E_{x}}\right)^{\prime}+\beta \mathbf{A}_{51}^{-1} \mathbf{A}_{52}\left(\mathbf{A}_{21}+\mathbf{A}_{22} \mathbf{A}_{51}^{-1} \mathbf{A}_{52}\right)^{-1} \mathbf{A}_{23} \mathbf{Y}^{H_{x}}
\end{aligned}
$$

\subsection{Method of solution}

In general the system (3.5.20) can be solved by the Finite Element method [18], [52]. It relies on a spatial discretization, i.e. divides the whole computational domain into a number of elements. On each of these elements the 
unknown function is represented as a superposition of some basis functions. The coefficients of the expansion are found using the weak form of eqn. (3.5.20). While this method is very general, it quickly introduces a large number of unknowns.

However, due to common techniques of fabrication many waveguides do not have a completely arbitrary refractive index distribution, but rather one which is piecewise constant along the horizontal axis or can be approximated as such. The waveguide then can be split in several vertical slices, where the refractive index does not change in the horizontal direction. In each of these layers the general solution of (3.5.20) can be written down analytically. Gluing them together across the vertical interfaces will give the desired mode profile.

In the following we will outline each of these methods in more detail.

\subsubsection{Arbitrary refractive index distribution: Finite Element Method}

In case of an arbitrary permittivity distribution $\varepsilon(x, y)$ (diffused waveguide, waveguide with slanted sidewalls) the matrices $\mathbf{S}$ depend on $y$, as their elements include overlap integrals with the permittivity $\varepsilon(x, y)$. One of the ways to solve the differential equation (3.5.20) is by using the Finite Element Method.

By multiplying both sides of (3.5.20) from the left by some continuous test vector-function $\mathbf{v}$ and integrating over $y$ one gets the weak form of equation (3.5.20):

$$
\int\left(-\mathbf{v}^{\top} \mathbf{S}_{1} \mathbf{u}+\left(\mathbf{v}^{\top}\right)^{\prime} \mathbf{S}_{2} \mathbf{u}^{\prime}\right) d y+\beta \int\left(\left(\mathbf{v}^{\top}\right)^{\prime} \mathbf{S}_{3} \mathbf{u}+\mathbf{v}^{\top} \mathbf{S}_{3} \mathbf{u}^{\prime}\right) d y+\beta^{2} \int \mathbf{v}^{\top} \mathbf{S}_{2} \mathbf{u} d y=0
$$

Then we expand the solution $\mathbf{u}$ into a finite combination of the basis functions $\boldsymbol{\varphi}_{i j}$,

$$
\mathbf{u}(y)=\sum_{i=1}^{n_{d}} \sum_{j=1}^{n_{g}} a_{i j} \boldsymbol{\varphi}_{i j}(y)
$$

with $n_{d}$ the dimension of the vector $\mathbf{u} ; n_{g}$ the number of consecutive grid 
points $y_{j}$ into which the $y$-axis has been divided, and

$$
\boldsymbol{\varphi}_{i j}(y)=\left(\begin{array}{c}
0 \\
\vdots \\
\hat{\varphi}_{j}(y) \\
\vdots \\
0
\end{array}\right) \leftarrow i^{\text {th }} \text { position }
$$

with, for example, linear basis functions

$$
\hat{\varphi}_{j}(y)= \begin{cases}0, & y<y_{j-1} \text { or } y \geq y_{j+1} ; \\ \frac{y-y_{j-1}}{y_{j}-y_{j-i}}, & y_{j-1} \leq y<y_{j} \\ \frac{y_{j+1}-y}{y_{j+1}-y_{j}}, & y_{j} \leq y<y_{j+1} .\end{cases}
$$

As eqn. (3.6.24) should hold for an arbitrary continuous $\mathbf{v}$, we choose it to be one of the basis functions $\varphi_{i j}$. For $i=1, \ldots, n_{d}$ and $j=1, \ldots, n_{g}$ this results in the system of exactly $n_{d} \cdot n_{g}$ linear equations

$$
\left(-\hat{\mathbf{S}}_{1}+\hat{\mathbf{S}}_{2}\right) \mathbf{a}+\beta\left(\hat{\mathbf{S}}_{3}+\hat{\mathbf{S}}_{5}\right) \mathbf{a}+\beta^{2} \hat{\mathbf{S}}_{4} \mathbf{a}=0,
$$

where $\mathbf{a}^{\boldsymbol{\top}}=\left(a_{i j}\right)=\left(\left[a_{11}, \ldots, a_{n_{d} 1}\right],\left[a_{12}, \ldots, a_{n_{d} 2}\right], \ldots,\left[a_{1 n_{g}}, \ldots, a_{n_{d} n_{g}}\right]\right)$ (the subscript ${ }_{i j}$ here refers to the $j^{\text {th }}$ element of the $i^{\text {th }}$ subvector). Since for any square matrix $\mathbf{M}$ of dimension $n_{d} n_{g} \times n_{d} n_{g}$

$$
\boldsymbol{\varphi}_{p m}^{\top} \mathbf{M} \boldsymbol{\varphi}_{i j}=\hat{\varphi}_{m}(y) \cdot M_{p i}(y) \cdot \hat{\varphi}_{j}
$$

holds, the matrices $\mathbf{S}$ turn to be of the following form

$$
\begin{aligned}
& \hat{\mathbf{S}}_{1}(p m, i j)=\int \hat{\varphi}_{m} S_{1 p i} \hat{\varphi}_{j} d y, \\
& \hat{\mathbf{S}}_{2}(p m, i j)=\int\left(\hat{\varphi}_{m}\right)^{\prime} S_{2 p i}\left(\hat{\varphi}_{j}\right)^{\prime} d y, \\
& \hat{\mathbf{S}}_{3}(p m, i j)=\int\left(\hat{\varphi}_{m}\right)^{\prime} S_{3 p i} \hat{\varphi}_{j} d y, \\
& \hat{\mathbf{S}}_{4}(p m, i j)=\int \hat{\varphi}_{m} S_{2 p i} \hat{\varphi}_{j} d y, \\
& \hat{\mathbf{S}}_{5}(p m, i j)=\int \hat{\varphi}_{m} S_{3 p i}\left(\hat{\varphi}_{j}\right)^{\prime} d y,
\end{aligned}
$$

where the indices $p m$ and $i j$ have the same meaning as in the definition of the vector a.

The solution of the quadratic eigenvalue problem (3.6.28) with $\beta$ as an eigenvalue can be found by transforming it to the linear eigenvalue problem

$$
\left(\begin{array}{cc}
\mathbf{0} & \mathbf{1} \\
-\hat{\mathbf{S}}_{4}^{-1}\left(-\hat{\mathbf{S}}_{1}+\hat{\mathbf{S}}_{2}\right) & -\hat{\mathbf{S}}_{4}^{-1}\left(\hat{\mathbf{S}}_{3}+\hat{\mathbf{S}}_{5}\right)
\end{array}\right)\left(\begin{array}{l}
\mathbf{a} \\
\mathbf{b}
\end{array}\right)=\beta\left(\begin{array}{l}
\mathbf{a} \\
\mathbf{b}
\end{array}\right) .
$$


This is a quite straightforward, but expensive approach, as the dimension of the transformed problem is doubled in comparison to the original one. Other more involved approaches to tackle a quadratic eigenvalue problem can be found e.g. in [98]. We apply standard general eigenvalue solvers as embedded within the LAPACK [58] package. Specialized solvers could be employed, provided that an initial guess for the propagation constant, or a range of possible eigenvalues, are available for the problem at hand. On the other hand, there are situations where all the propagation constants $\beta$ and corresponding functions $\mathbf{u}$ need to be found together, e.g. if one wants to expand a 3D field in terms of vectorial modes of some channel waveguide, as required for the implementation of transparent boundary conditions (cf. Chapter [5, Ref. [48]).

\subsubsection{Piecewise constant refractive index distribution}

If a waveguide has a piecewise constant rectangular refractive index profile, it can be divided by vertical lines into slices with constant refractive index distribution along the $y$-direction. In each of these slices the matrices $\mathbf{S}$ do not depend on $y$. Then (3.5.20) can be rewritten in a more familiar manner: Inside each of the slices $\mathbf{u}$ should satisfy a system of second order differential equations with constant coefficients $\mathbf{S}$ and a parameter $\beta^{2}$

$$
\mathbf{S}_{1} \mathbf{u}+\mathbf{S}_{2} \mathbf{u}^{\prime \prime}=\beta^{2} \mathbf{S}_{2} \mathbf{u}
$$

together with the continuity conditions (3.5.22). Moreover the matrices $\mathbf{S}_{1}$ and $\mathbf{S}_{2}$ are block-diagonal in such a way that the equations for the functions $\mathbf{Y}^{E_{x}}$ and $\mathbf{Y}^{H_{x}}$ decouple inside each of the slices; coupling occurs only across the vertical interfaces.

Inside each slice a particular solution of the system (3.6.32) can be readily written as

$$
\mathbf{u}=c \mathrm{e}^{\alpha y} \mathbf{p}
$$

with some constants $c, \alpha$ and a vector p. By substituting (3.6.33) into (3.6.32) we find a generalized eigenvalue problem with $\eta^{2}=\beta^{2}-\alpha^{2}$ as an eigenvalue:

$$
\mathbf{S}_{1} \mathbf{p}=\eta^{2} \mathbf{S}_{2} \mathbf{p}
$$

So inside each of the slices with uniform permittivity along the $y$-axis 
the function $\mathbf{u}$ can be represented as

$$
\mathbf{u}=\sum_{j}\left(c_{1 j} \mathrm{e}^{\sqrt{\beta^{2}-\eta_{j}^{2}} y}+c_{2 j} \mathrm{e}^{-\sqrt{\beta^{2}-\eta_{j}^{2}} y}\right) \mathbf{p}_{j}
$$

with eigenvalues $\eta_{j}$ and corresponding eigenvectors $\mathbf{p}_{j}$ from (3.6.34).

By matching the solutions of the each individual slab across the vertical interfaces using (3.5.22) and looking only for exponentially decaying solutions for $y \rightarrow \pm \infty$, one can obtain an eigenvalue problem

$$
\mathbf{M}(\beta) \mathbf{c}=0 .
$$

The vector $\mathbf{c}$ consists of all unknown coefficients $c_{1 j}$ and $c_{2 j}$ from the representations of $\mathbf{u}(3.6 .35)$ on all individual slices. The matrix $\mathbf{M}$ depends on $\beta$ in a non-linear, even non-polynomial way. One of the strategies to tackle this is at first to specify a range of admissible values $\beta \in\left[I_{1}, I_{2}\right]$, where solutions $\beta$ are sought. As we are looking only for propagating modes, with decaying field (3.6.35) at $y \rightarrow \pm \infty, I_{1}$ should be not smaller than the biggest eigenvalue $\eta_{j}$ of (3.6.34) in the left-most and the right-most slabs. At the same time we require that there exists at least one oscillating function in at least one vertical slab. So $I_{2}$ should be smaller than the biggest eigenvalue $\eta_{j}$ of (3.6.34) of all the constituting slabs, except the left- and the rightmost ones. Once this interval is at hand, we scan through it looking for a $\beta$ such that the matrix $\mathbf{M}(\beta)$ has at least one zero eigenvalue. Obviously, to find a non-trivial solution with certain accuracy requires some iterations. Moreover a large step size might lead to missing some roots while scanning the interval.

Once a nontrivial solution $\beta$, $\mathbf{c}$ of (3.6.36) is at hand, $\mathbf{u}$ can be reconstructed using (3.6.35). And then all field components can be obtained according to expressions (3.5.23) together with (3.4.14) or (3.4.15).

\subsection{Relation with the Effective Index Method}

In the following section we are going to show what happens if only a single, TE or TM, slab mode is taken into account in VEIM5 (3.4.14). Using the variational reasoning we will rigorously derive an analog to the Effective Index method. 


\subsubsection{TE polarization}

Let us take only one TE slab mode with propagation constant $\beta_{\mathrm{r}}$ from a reference slice $\mathrm{r}$ with permittivity distribution $\varepsilon_{\mathrm{r}}(x)$, and use it to represent the vectorial field profile of the complete waveguide as in eqn. (3.4.14). Due to the fact that $X^{E_{x}} \equiv 0$, according to (3.5.20) the unknown function $Y^{H_{x}}$ satisfies the eqn.

$$
\begin{aligned}
A_{41} Y^{H_{x}}+ & \left(-\mathrm{i} A_{42}\left(A_{21}+A_{22} A_{51}^{-1} A_{52}\right)^{-1} A_{23}\left(Y^{H_{x}}\right)^{\prime}\right)^{\prime}= \\
= & \beta^{2}\left(-\mathrm{i} A_{42}\left(A_{21}+A_{22} A_{51}^{-1} A_{52}\right)^{-1} A_{23}\right) Y^{H_{x}}
\end{aligned}
$$

After some manipulations, using the relations between the modal components $\chi^{E_{y}}, \chi^{H_{x}}$ and $\chi^{H_{z}}$ of the slab mode the above relation can be rewritten as follows

$$
\left(\frac{1}{\varepsilon_{\mathrm{eff}}}\left(Y^{H_{x}}\right)^{\prime}\right)^{\prime}+k^{2} Y^{H_{x}}=\beta^{2} \frac{1}{\varepsilon_{\mathrm{eff}}} Y^{H_{x}}
$$

with

$$
\varepsilon_{\mathrm{eff}}(y)=\frac{\beta_{\mathrm{r}}^{2}}{k^{2}}+\frac{\left\langle\chi^{E_{y}},\left(\varepsilon(x, y)-\varepsilon_{\mathrm{r}}(y)\right) \chi^{E_{y}}\right\rangle}{\left\langle\chi^{E_{y}}, \chi^{E_{y}}\right\rangle} .
$$

This looks exactly as a TM mode equation, similar to the standard Effective Index Method. In the reference slice one has $\varepsilon=\varepsilon_{\mathrm{r}}$, and the effective permittivity $\varepsilon_{\text {eff }}$ is equal to the squared effective index of the mode of the reference slice $\beta_{\mathrm{r}}^{2} / k^{2}$. In other slices this squared effective index is modified by the difference between the local permittivity and that of the reference slice, weighted by the local intensity of the fundamental component of the reference mode profile. Hence, on the contrary to the EIM, even in slices where no guided mode exist, the effective permittivity can still be defined.

Now it is instructive to see how the mode profile adjusts both in the reference slabs and elsewhere. Inside a slice with constant permittivity $\varepsilon_{\text {eff }}$, eqn. (3.7.38) permits solutions of the form

$$
Y^{H_{x}}=c_{+} \mathrm{e}^{\mathrm{i} \alpha y}+c_{-} \mathrm{e}^{-\mathrm{i} \alpha y}
$$

for arbitrary constants $c_{+}$and $c_{-}$and with

$$
\beta^{2}+\alpha^{2}=k^{2} \varepsilon_{\text {eff }}
$$


With the abbreviation $\rho^{2}=k^{2} \varepsilon_{\text {eff }}$ from (3.5.23) it follows that

$$
\begin{aligned}
Y^{H_{z}} & =\frac{\beta_{\mathrm{r}} \beta}{\rho^{2}}\left(c_{+} \mathrm{e}^{\mathrm{i} \alpha y}+c_{-} \mathrm{e}^{-\mathrm{i} \alpha y}\right), \\
Y^{E_{y}} & =Y^{H_{z}}, \\
Y^{E_{z}} & =\frac{\beta_{\mathrm{r}} \alpha}{\rho^{2}}\left(c_{+} \mathrm{e}^{\mathrm{i} \alpha y}-c_{-} \mathrm{e}^{-\mathrm{i} \alpha y}\right), \\
Y^{H_{y}} & =-Y^{E_{z}} .
\end{aligned}
$$

By introducing an angle $\theta$ such that $\cos \theta=\beta / \rho$, one can write

$$
\begin{aligned}
\left(\begin{array}{c}
Y^{E_{x}}, Y^{E_{y}}, Y^{E_{z}} \\
Y^{H_{x}}, Y^{H_{y}}, Y^{H_{z}}
\end{array}\right)(y) & =c_{+} \frac{\beta_{\mathrm{r}}}{\rho} \mathrm{e}^{\mathrm{i} \rho \sin \theta y}\left(\begin{array}{crr}
0, & \cos \theta, & \sin \theta \\
\rho / \beta_{\mathrm{r}}, & -\sin \theta, & \cos \theta
\end{array}\right)+ \\
& +c_{-} \frac{\beta_{\mathrm{r}}}{\rho} \mathrm{e}^{-\mathrm{i} \rho \sin \theta y}\left(\begin{array}{ccc}
0, & \cos \theta, & -\sin \theta \\
\rho / \beta_{\mathrm{r}}, & \sin \theta, & \cos \theta
\end{array}\right) .
\end{aligned}
$$

If we use the principal square roots of $\alpha^{2}$ and $\rho^{2}$ for $\alpha$ and $\rho$, and the principal inverse cosine for $\theta$ eq. (3.7.43) can be interpreted as follows. In the slice where the reference slab mode lives $\rho=\beta_{\mathrm{r}}$, and we find that functions $Y$ act as a rotation of the slab mode, such that the projection of the propagation constant of this mode onto the $z$-axis will match the global propagation constant $\beta$. In other slices, in addition to the rotation of the $y$ and $z$ components of the slab mode, the $x$ component is scaled by $\rho / \beta_{\mathrm{r}}$.

\subsubsection{TM polarization}

Analogously, the eqn. (3.5.20) can be rewritten for a single TM mode, with a field template as in (3.4.14). We now have $X^{H_{x}} \equiv 0$ in eqn. (3.5.20) and using the properties of the TM slab mode, the original equation for the unknown function $Y^{E_{x}}$,

$$
\begin{aligned}
A_{11} Y^{E_{x}}+ & \left(-\mathrm{i} A_{12}\left(A_{51}+A_{52} A_{21}^{-1} A_{22}\right)^{-1} A_{53}\left(Y^{E_{x}}\right)^{\prime}\right)^{\prime}= \\
& =\beta^{2}\left(-\mathrm{i} A_{12}\left(A_{51}+A_{52} A_{21}^{-1} A_{22}\right)^{-1} A_{53}\right) Y^{E_{x}}
\end{aligned}
$$

can be rewritten as

$$
\left(\frac{1}{\varepsilon_{1 \mathrm{eff}}}\left(Y^{E_{x}}\right)^{\prime}\right)^{\prime}+k^{2} \varepsilon_{2} Y^{E_{x}}=\beta^{2} \frac{1}{\varepsilon_{1 \mathrm{eff}}} Y^{E_{x}},
$$


with

$$
\begin{aligned}
& \varepsilon_{1 \mathrm{eff}}(y)=\frac{\beta_{\mathrm{r}}^{2}}{k^{2}} \frac{\left\langle\chi^{E_{z}}, \varepsilon_{\mathrm{r}}(x) \chi^{E_{z}}\right\rangle}{\left\langle\chi^{E_{z}}, \varepsilon(x, y) \chi^{E_{z}}\right\rangle}+\frac{\left\langle\chi^{H_{y}}, \chi^{H_{y}}\right\rangle}{\left\langle\chi^{H_{y}}, \frac{1}{\varepsilon_{\mathrm{r}}(x)} \chi^{H_{y}}\right\rangle} \frac{\left\langle\chi^{E_{z}},\left(\varepsilon(x, y)-\varepsilon_{\mathrm{r}}(x)\right) \chi^{E_{z}}\right\rangle}{\left\langle\chi^{E_{z}}, \varepsilon(x, y) \chi^{E_{z}}\right\rangle}, \\
& \varepsilon_{2}(y)=\frac{\left\langle\chi^{E_{x}}, \varepsilon(x, y) \chi^{E_{x}}\right\rangle}{\left\langle\chi^{E_{x}}, \varepsilon_{\mathrm{r}}(x) \chi^{E_{x}}\right\rangle} .
\end{aligned}
$$

This appears to be neither a standard TE nor a TM mode equation, but something in between, with the local refractive index distribution appearing both in the terms with and without derivative. In the reference slice with $\varepsilon=\varepsilon_{\mathrm{r}}$, the effective permittivity $\varepsilon_{1 \text { eff }}$ is equal to the squared effective index $\beta_{\mathrm{r}}^{2} / k^{2}$ of the mode of the reference slice and $\varepsilon_{2}=1$. Contrary to the EIM, even in slices where no guided mode exists quantities that act like effective indices can still be rigorously defined.

What concerns the mode profile, in intervals along the $y$-axis with constant $\varepsilon_{1 \text { eff }}$ and $\varepsilon_{2}$, local solutions of eqn. (3.7.45) are of the form

$$
Y^{E_{x}}=c_{+} \mathrm{e}^{\mathrm{i} \alpha y}+c_{-} \mathrm{e}^{-\mathrm{i} \alpha y}
$$

with

$$
\beta^{2}+\alpha^{2}=k^{2} \varepsilon_{1 \mathrm{eff}} \varepsilon_{2}
$$

Let us denote the right hand side of eqn. (3.7.48) as $\rho^{2}=k^{2} \varepsilon_{1 \text { eff }} \varepsilon_{2}$ and

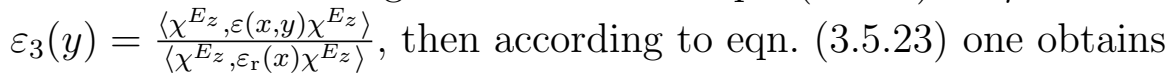

$$
\begin{aligned}
Y^{H_{z}} & =\frac{\beta_{\mathrm{r}} \alpha \varepsilon_{2}}{\rho^{2}}\left(c_{+} \mathrm{e}^{\mathrm{i} \alpha y}-c_{-} \mathrm{e}^{-\mathrm{i} \alpha y}\right), \\
Y^{E_{y}} & =-\frac{1}{\varepsilon_{3}} Y^{H_{z}}, \\
Y^{H_{y}} & =\frac{\beta_{\mathrm{r}} \beta \varepsilon_{2}}{\rho^{2}}\left(c_{+} \mathrm{e}^{\mathrm{i} \alpha y}+c_{-} \mathrm{e}^{-\mathrm{i} \alpha y}\right), \\
Y^{E_{z}} & =\frac{1}{\varepsilon_{3}} Y^{H_{y}}
\end{aligned}
$$


By introducing an angle $\theta$ such that $\cos \theta=\beta / \rho$, one can write

$$
\begin{aligned}
& \left(\begin{array}{r}
Y^{E_{x}}, Y^{E_{y}}, Y^{E_{z}} \\
Y^{H_{x}}, Y^{H_{y}}, Y^{H_{z}}
\end{array}\right)(y)= \\
& =c_{+} \frac{\beta_{\mathrm{r}} \varepsilon_{2}}{\rho} \mathrm{e}^{\mathrm{i} \rho \sin \theta y}\left(\begin{array}{lrr}
\rho / \beta_{\mathrm{r}} \varepsilon_{2}, & -\varepsilon_{3}^{-1} \sin \theta, & \varepsilon_{3}^{-1} \cos \theta \\
0, & \cos \theta, & \sin \theta
\end{array}\right)+ \\
& \quad+c_{-} \frac{\beta_{\mathrm{r}} \varepsilon_{2}}{\rho} \mathrm{e}^{-\mathrm{i} \rho \sin \theta y}\left(\begin{array}{lrr}
\rho / \beta_{\mathrm{r}} \varepsilon_{2}, & \varepsilon_{3}^{-1} \sin \theta, & \varepsilon_{3}^{-1} \cos \theta \\
0, & \cos \theta, & -\sin \theta
\end{array}\right)
\end{aligned}
$$

In the reference slice $\rho=\beta_{\mathrm{r}}$ and we find that the functions $Y$ also in this case act as a rotation of the slab mode. In all other slices, while the $y$ and $z$-components of the magnetic field are just rotated by the angle $\theta$, the electric $y$ and $z$ components are not only rotated, but also scaled by $\varepsilon_{3}^{-1}$. In addition to this the $x$-component is scaled by $\rho / \beta_{\mathrm{r}} \varepsilon_{2}$.

\subsection{Relation with the Film Mode Matching Method}

As we could see in the previous section if only one, TE or TM, slab mode is used to expand the total field profile using the 5 component expansion (3.4.14), the variational procedure leads to functions $Y$ that act as a rotation. Then the field representation inside the slice where the slab mode lives replicates the field ansatz of the FMM (cf. section 3.4). In the following we look at the case when multiple TE and TM slab modes appear in the 5 component expansion (3.4.14).

Let us rewrite the second, third, fifth and sixth equations of (3.5.16) as

$$
\begin{aligned}
& \mathbf{I}_{\mathrm{TE}}\left(\mathbf{Y}_{\mathrm{TE}}^{E_{y}}-\mathbf{Y}_{\mathrm{TE}}^{H_{z}}\right)+\mathbf{I}_{\mathrm{TM}}\left(\mathbf{Y}_{\mathrm{TM}}^{E_{y}}+\mathbf{Y}_{\mathrm{TM}}^{H_{z}}\right)=\mathbf{A}_{21}^{-1} \mathbf{A}_{23}\left(\beta \mathbf{Y}^{H_{x}}-\mathbf{G}_{\mathrm{TE}} \mathbf{Y}_{\mathrm{TE}}^{H_{z}}\right) \\
& -\mathbf{I}_{\mathrm{TE}}\left(\mathbf{Y}_{\mathrm{TE}}^{E_{y}}-\mathbf{Y}_{\mathrm{TE}}^{H_{z}}\right)+\mathbf{I}_{\mathrm{TM}}\left(\mathbf{Y}_{\mathrm{TM}}^{E_{y}}+\mathbf{Y}_{\mathrm{TM}}^{H_{z}}\right)=\mathbf{A}_{51}^{-1} \mathbf{A}_{53}\left(\mathbf{G}_{\mathrm{TM}} \mathbf{Y}_{\mathrm{TM}}^{E_{y}}-\mathrm{i}\left(\mathbf{Y}^{E_{x}}\right)^{\prime}\right) \\
& \mathbf{I}_{\mathrm{TE}}\left(\mathbf{Y}_{\mathrm{TE}}^{E_{z}}+\mathbf{Y}_{\mathrm{TE}}^{H_{y}}\right)+\mathbf{I}_{\mathrm{TM}}\left(\mathbf{Y}_{\mathrm{TM}}^{E_{z}}-\mathbf{Y}_{\mathrm{TM}}^{H_{y}}\right)=\mathbf{A}_{21}^{-1} \mathbf{A}_{23}\left(\mathbf{G}_{\mathrm{TE}} \mathbf{Y}_{\mathrm{TE}}^{H_{y}}-\mathrm{i}\left(\mathbf{Y}_{x}^{H_{x}}\right)^{\prime}\right) \\
& \mathbf{I}_{\mathrm{TE}}\left(\mathbf{Y}_{\mathrm{TE}}^{E_{z}}+\mathbf{Y}_{\mathrm{TE}}^{H_{y}}\right)-\mathbf{I}_{\mathrm{TM}}\left(\mathbf{Y}_{\mathrm{TM}}^{E_{z}}-\mathbf{Y}_{\mathrm{TM}}^{H_{y}}\right)=\mathbf{A}_{51}^{-1} \mathbf{A}_{53}\left(\beta \mathbf{Y}^{E_{x}}-\mathbf{G}_{\mathrm{TM}} \mathbf{Y}_{\mathrm{TM}}^{E_{z}}\right)
\end{aligned}
$$

with functions $\mathbf{Y}_{b}^{a}$ corresponding to a vector of all the functions $Y$ related to modal component of polarization $b$, used to expand component $a$ of the total field. $\mathbf{G}_{b}$ is a diagonal matrix with propagation constants $\beta_{\mathrm{r}, j}$ of the 
slab modes of polarization $b$ sitting on the diagonal. Matrices

$$
\mathbf{I}_{\mathrm{TE}}=\left(\begin{array}{c}
\mathbf{1}_{n_{\mathrm{TE}} \times n_{\mathrm{TE}}} \\
\mathbf{0}_{n_{\mathrm{TM}} \times n_{\mathrm{TE}}}
\end{array}\right), \quad \mathbf{I}_{\mathrm{TM}}=\left(\begin{array}{c}
\mathbf{0}_{n_{\mathrm{TE}} \times n_{\mathrm{TM}}} \\
\mathbf{1}_{n_{\mathrm{TM}} \times n_{\mathrm{TM}}}
\end{array}\right)
$$

have been introduced to increase the readability of the equations. Here $\mathbf{1}_{d}$ and $\mathbf{0}_{d}$ denote correspondingly the unity- and zero-matrix of a dimension $d$, and symbols $n_{\mathrm{TE}}$ and $n_{\mathrm{TM}}$ denote the number of slab modes of respectively TE and TM polarization included in the expansion (3.4.14).

Obviously, functions $\mathbf{Y}$ that satisfy

$$
\begin{array}{ll}
\mathbf{Y}_{\mathrm{TE}}^{E_{y}}=\mathbf{Y}_{\mathrm{TE}}^{H_{z}}, & \mathbf{Y}_{\mathrm{TM}}^{E_{y}}=-\mathbf{Y}_{\mathrm{TM}}^{H_{z}}, \\
\beta \mathbf{Y}^{H_{x}}=\mathbf{G}_{\mathrm{TE}} \mathbf{Y}_{\mathrm{TE}}^{H_{z}}, & \mathbf{G}_{\mathrm{TM}} \mathbf{Y}_{\mathrm{TM}}^{E_{y}}=\mathrm{i}\left(\mathbf{Y}^{E_{x}}\right)^{\prime}, \\
\mathbf{Y}_{\mathrm{TE}}^{E_{z}}=-\mathbf{Y}_{\mathrm{TE}}^{H_{y}}, & \mathbf{Y}_{\mathrm{TM}}^{E_{z}}=\mathbf{Y}_{\mathrm{TM}}^{H_{y}}, \\
\mathbf{G}_{\mathrm{TE}} \mathbf{Y}_{\mathrm{TE}}^{H_{y}}=\mathrm{i}\left(\mathbf{Y}^{H_{x}}\right)^{\prime}, & \beta \mathbf{Y}^{E_{x}}=\mathbf{G}_{\mathrm{TM}} \mathbf{Y}_{\mathrm{TM}}^{E_{z}}
\end{array}
$$

are solutions of (3.8.51). Using these relations together with the first and the fourth equations of (3.5.16) result in

$$
\begin{aligned}
& \left(\mathbf{Y}^{E_{x}}\right)^{\prime \prime}+\left(\mathbf{G}_{\mathrm{TM}}\right)^{2} \mathbf{Y}^{E_{x}}=\beta^{2} \mathbf{Y}^{E_{x}} \\
& \left(\mathbf{Y}^{H_{x}}\right)^{\prime \prime}+\left(\mathbf{G}_{\mathrm{TE}}\right)^{2} \mathbf{Y}^{H_{x}}=\beta^{2} \mathbf{Y}^{H_{x}}
\end{aligned}
$$

According to eqns. (3.8.51) and (3.8.54) all the functions $Y$ decouple inside the slice where the slab modes belongs to. So we can solve these equations for all the components of $\mathbf{Y}^{E_{x}}$ and $\mathbf{Y}^{H_{x}}$ separately. Solutions of (3.8.54) have the form

$$
Y_{j}^{H_{x}}=c_{+, j} \mathrm{e}^{\mathrm{i} \alpha_{j} y}+c_{-, j} \mathrm{e}^{-\mathrm{i} \alpha_{j} y}
$$

with

$$
\beta^{2}+\alpha_{j}^{2}=\beta_{\mathrm{r}, j}^{2} .
$$

Other components can be derived from (3.8.51) as

$$
\begin{aligned}
\left(\begin{array}{c}
Y_{j}^{E_{x}}, Y_{j}^{E_{y}}, Y_{j}^{E_{z}} \\
Y_{j}^{H_{x}}, Y_{j}^{H_{y}}, Y_{j}^{H_{z}}
\end{array}\right)(y) & =c_{+, j} \mathrm{e}^{\mathrm{i} \beta_{\mathrm{r}, j} \sin \theta_{j} y}\left(\begin{array}{rrr}
0, & \cos \theta_{j}, & \sin \theta_{j} \\
1, & -\sin \theta_{j}, & \cos \theta_{j}
\end{array}\right)+ \\
& +c_{-, j} \mathrm{e}^{-\mathrm{i} \beta_{\mathrm{r}, j} \sin \theta_{j} y}\left(\begin{array}{rrr}
0, & \cos \theta_{j}, & -\sin \theta_{j} \\
1, & \sin \theta_{j}, & \cos \theta_{j}
\end{array}\right),
\end{aligned}
$$

where $\cos \theta_{j}=\beta / \beta_{\mathrm{r}, j}$. 
Hence the functions $Y_{j}$ corresponding to TE slab mode number $j$ rotate the original slab mode around the $x$-axis such that the projection of its propagation constant $\beta_{\mathrm{r}, j}$ onto the direction of propagation $z$ will be precisely the propagation constant $\beta$ of the mode of the complete waveguide structure. The same is true for TM slab modes.

We showed that the field ansatz of rotated slab modes, as used locally in the Film Mode Matching method [95], [5] can be found also by the present approach where it appears to be optimal. While in itself it might seem rather pointless to reinvent the method, the idea behind the present technique might be used in deriving some sort of analogue of the FMM for full 3D scattering problems, in which the structure varies in all 3 directions (cf. Chapter [5, Ref. [48]).

\section{$3.9 \quad$ Numerical results}

We will illustrate the method with four examples. The first two deal with waveguides with piecewise constant rectangular refractive index distribution. The third example is a waveguide with slanted sidewall and the fourth is an indiffused waveguide. We will use the acronym VEIM (variational effective index method) for results of the technique as introduced in sections $3.2-3.8$.

\subsubsection{Box-shaped waveguide}

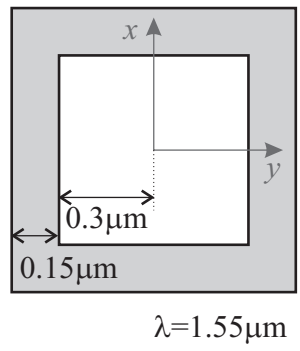

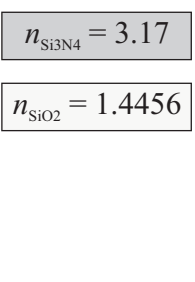

$n_{\mathrm{Si} 3 \mathrm{~N} 4}=3.17$
$n_{\mathrm{SiO} 2}=1.4456$

Figure 3.4: Structure of the Box-Shaped Waveguide. The vertical extents of the computational window range from $-2.5 \mu \mathrm{m}$ to $2.5 \mu \mathrm{m}$.

Consider the box-shaped waveguide of Figure 3.4, originating from [66]. It can be divided into five vertical slices with three distinct cross-sections (Figure 3.5, left). We take slab modes from the side walls of the box (Figure 3.5, middle) to approximate the modal field in the entire cross-section (Figure 3.5, right). The waveguide will be analyzed with both 3 (3.4.15) and 
5 (3.4.14) component approximations, denoted by VEIM $3_{a, b}$ andVEIM $5_{a, b}$, where $a$ and $b$ are the number of TE and TM slab modes taken into account.

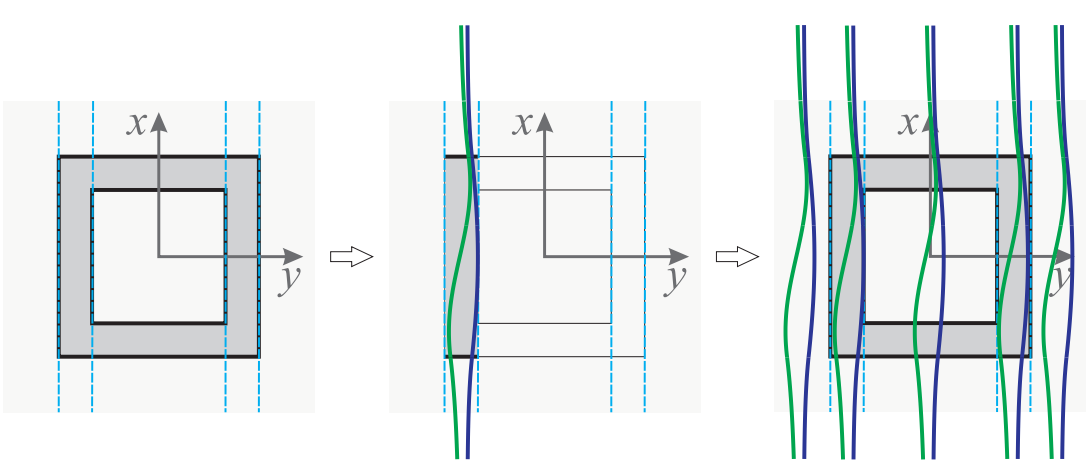

Figure 3.5: Subdivision of the waveguide into slices. Slab modes of the side walls are used to approximate the field of the mode everywhere.

In Figure 3.6 VEIM5 $5_{1,0}$ approximation of the vectorial mode profile of the fundamental TE-like mode is shown. In this case $\chi^{E_{y}}$ is multiplied by $Y^{E_{y}}$ and $Y^{E_{z}}$ to get $E_{y}$ and $E_{z}$ respectively; $\chi^{H_{x}}$ is multiplied by $Y^{H_{x}}$ to get $H_{x}$; and $\chi^{H_{z}}$ is multiplied by $Y^{H_{y}}$ and $Y^{H_{z}}$ to get $H_{y}$ and $H_{z}$ respectively. The figure contains plots of all contributing functions. Consistent with the observation in sec. 3.7.1, we see that $Y^{E_{y}}=Y^{H_{z}}$ and $Y^{E_{z}}=-Y^{H_{y}}$. Note that, contrary to the EIM, the field profile can still be visualized even when no local guided slab mode exists. 
(a)
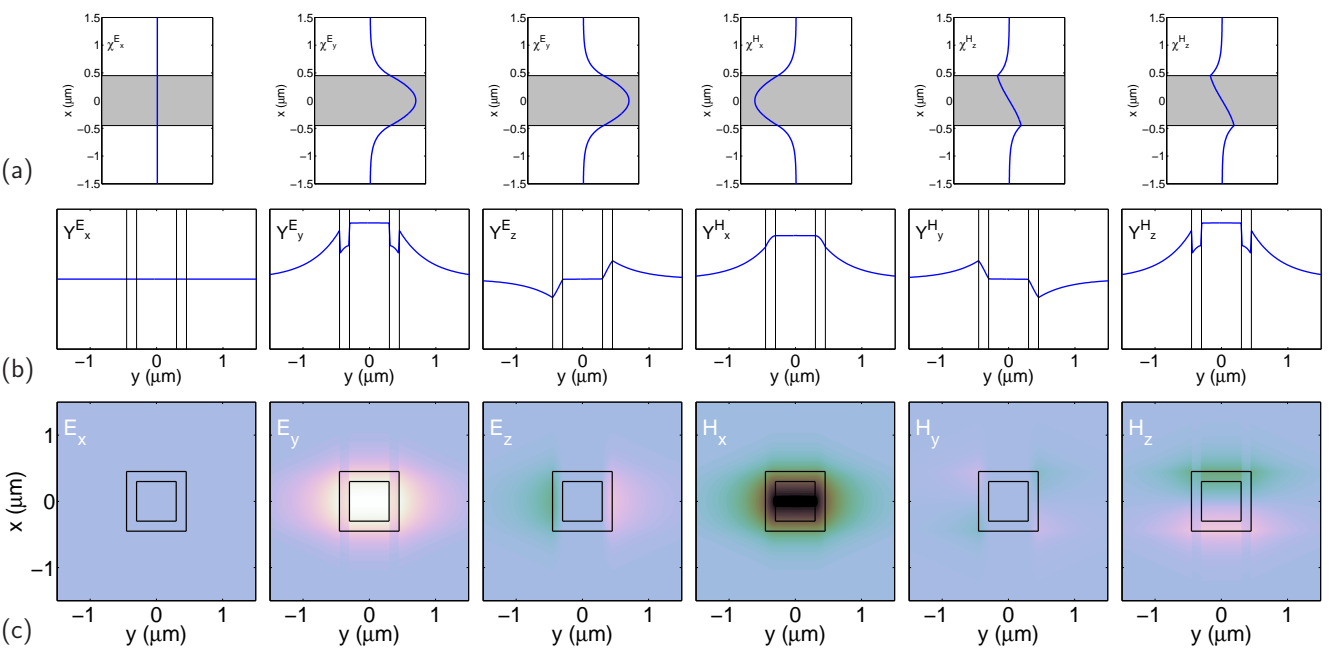

Figure 3.6: Square waveguide: (a) Functions $\chi$ in expansion VEIM51,0; (b) Functions $Y$ in expansion VEIM5 $5_{1,0}$; (c) Vectorial field profile VEIM5 $5_{1,0}$.

Next, Figure 3.7 gives an impression of the "converged" field profile obtained using VEIM3 $3_{30,30}$. The slab mode basis has been discretized by Dirichlet boundary conditions on the boundaries of the vertical computational window as given in Figure 3.4. Comparison with Figure 3.6 shows that even with a single mode in the representation, the main features of the true field profile are already visible. So the present method with one mode in the expansion can very well serve as a quick tool for qualitative analysis of the waveguide structures, while also being able to quantitatively analyze the waveguide by using more modes in the expansion.
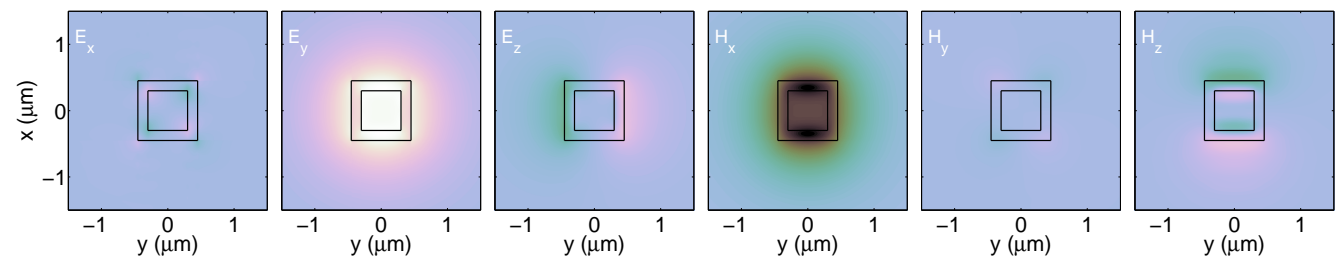

Figure 3.7: "Converged" (VEIM330,30) vectorial field profiles of the fundamental TE-like mode.

Figure 3.8 shows the propagation constant of the fundamental modes of the waveguide versus the number $m$ of TE and TM modes in the expansion 
VEIM $3_{m, m}$ for both the present method and a commercial FMM solver [10]. Both methods converge to the same value with comparable convergence speed.

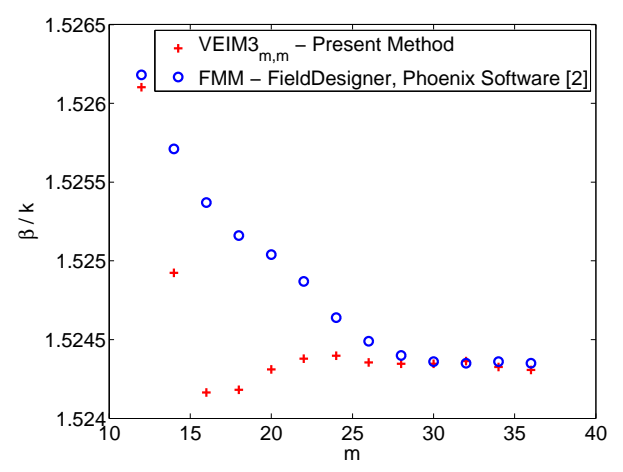

Figure 3.8: Convergence of the effective index of the fundamental TE-like mode of the box-shaped waveguide Figure 3.4 .

\subsubsection{Rectangular rib waveguide}
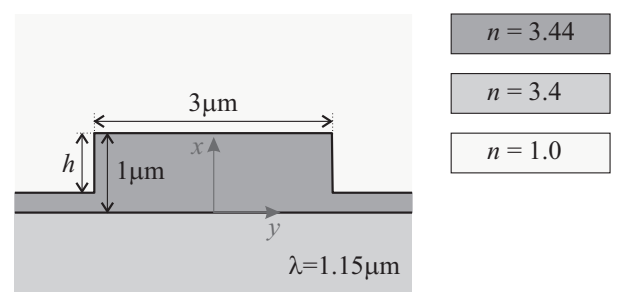

Figure 3.9: Structure of the Rib Waveguide. Vertical extents of the computational window are $[-2,2] \mu m$.

In this section we consider the rib structure from Figure 3.9, which is used as a benchmark waveguide in [102], [32], [60], and Chapter 2 of this thesis. The structure supports a fundamental TE and TM mode for all etch depths $h$ in the range we look in, which is $[0.2,1]$. The modes are strongly polarized, and thus it may be expected that an expansion using only TE or only TM modes (similar to a semi-vectorial calculation) will give good results.

At etch depths greater than $0.5 \mu \mathrm{m}$ guided modes do not exist outside the central slice, so the EIM fails to uniquely determine the effective refractive index of those regions. We analyze this structure with both 3- (3.4.15) and 5-component (3.4.14) approximations. In the following figures we will refer to them as VEIM $3_{a, b, c, d}$ and VEIM $5_{a, b, c, d}$ correspondingly. The subscript letters stand for number of slab modes used in the current approximation: $a$ - number of TE modes from the central slice, $b$ - number of TE modes 
from the outer slice, $c$ - number of TM modes from the central slice, $d-$ number of TM modes from the outer slice.

The slab modes are calculated using Dirichlet boundary conditions on the upper and lower computational domain boundaries. Because of this, the outer slice mode is still defined when the guided mode of that slice goes below cut-off.

Figure 3.10 and Figure 3.11 show plots of the TE and TM effective indices correspondingly using these different expansions versus the etch depth. The figures also show the corresponding EIM results, and, as reference, FMM results obtained by the commercial mode solver [10].

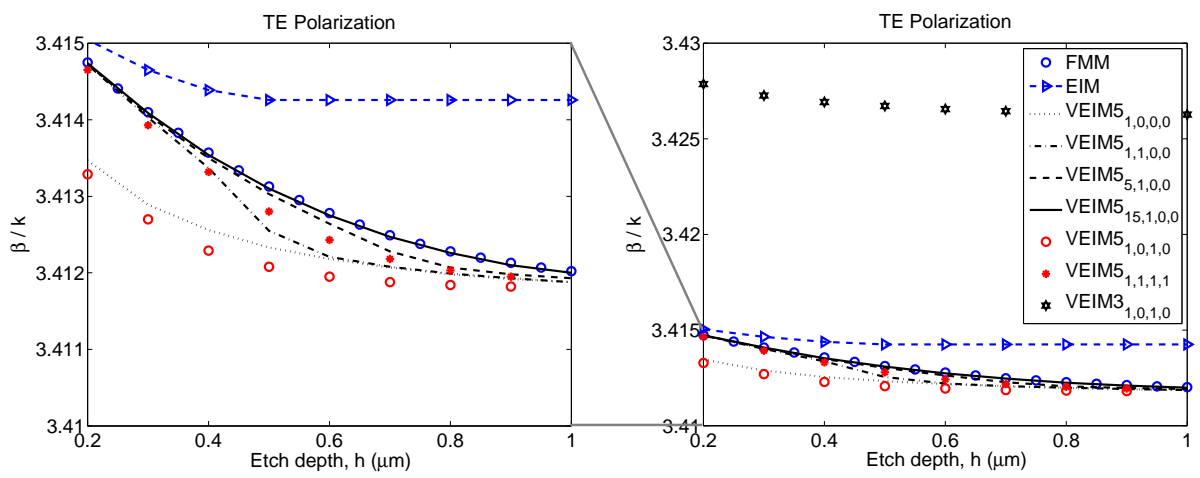

Figure 3.10: Convergence of the effective index $(\beta / k)$ of the fundamental TE mode of the rib waveguide.

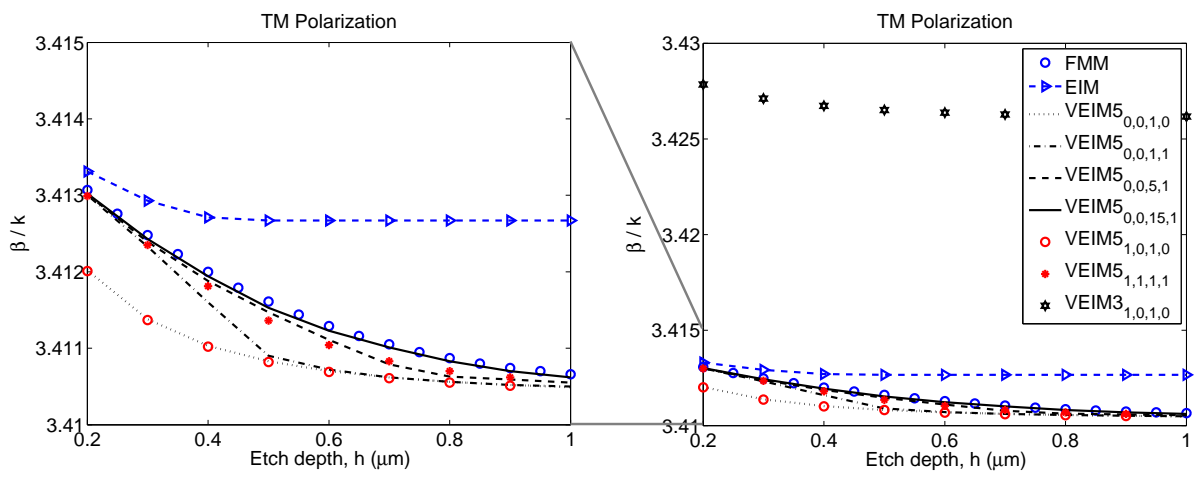

Figure 3.11: Convergence of the effective index $(\beta / k)$ of the fundamental TM mode of the rib waveguide. 
Comparing the results of our method with only one TE (VEIM $\left.5_{1,0,0,0}\right)$ or TM (VEIM $5_{0,0,1,0}$ ) mode of the central slice in the expansion (3.4.14) to the EIM results, shows that for larger etch depths, our results are much closer to the reference results - especially after the outer slice has gone below cutoff and the EIM uses the substrate refractive index as (constant) outer slice effective index.

Adding one outer slice mode to the VEIM expansion greatly improves its accuracy, especially if it is a guided slab mode; the VEIM $5_{1,1,0,0}$ curves are much closer to the reference results than the VEIM $5_{1,0,0,0}$ curves, especially at etch depths below $0.5 \mu \mathrm{m}$.

Taking five inner and one outer slice mode VEIM $5_{5,1,0,0}$ moves the results closer to the reference curve, while fifteen inner and one outer slice modes VEIM515,1,0,0 yield results that almost coincide with the reference. Note that these results use only TE or only TM modes in the 5-component expansion (3.4.14), i.e. the resulting fields are semi-vectorial; apparently a semivectorial approximation is sufficient for an accurate estimation of the effective indices of this structure.

The present method when using just one central slice TE and TM mode simultaneously with the three-component-per-mode approximation VEIM $3_{1,0,1,0}(3.4 .15)$ yields results that are quite far from the reference data. Moving to the five-component-per-mode approximation VEIM $5_{1,0,1,0}$ (3.4.14), on the other hand, gives much better results. Moreover, adding outer slice TE and TM modes VEIM $5_{1,1,1,1}$ greatly improves the estimation of propagation constant for both, TE and TM, polarizations.

\subsubsection{Waveguide with non-rectangular piecewise con- stant cross-section}

The waveguide cross-section of Figure 3.12 is part of a polarization rotator in $\mathrm{InP} / \mathrm{InGaAsP}$, proposed in [70]. Due to its slanted sidewall, the modes of this structure are highly hybrid.

Because of the slanted sidewall, the finite element scheme is more suitable to calculate the modes of this structure; the semi-analytical method requires a rather large number of slices, while the finite elements automatically take the slant into account. 


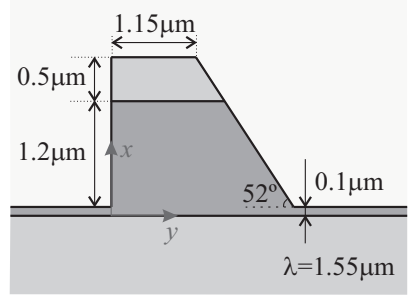

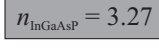

$n_{\mathrm{InP}}=3.17$

$n_{\text {air }}=1.0$

Figure 3.12: Structure of polarization converter from [70]. The computational window in the calculations is $(x, y) \in[-2,2.5] \times$ $[-2,3.5] \mu \mathrm{m}^{2} ; 50$ elements are used in the finite element scheme.
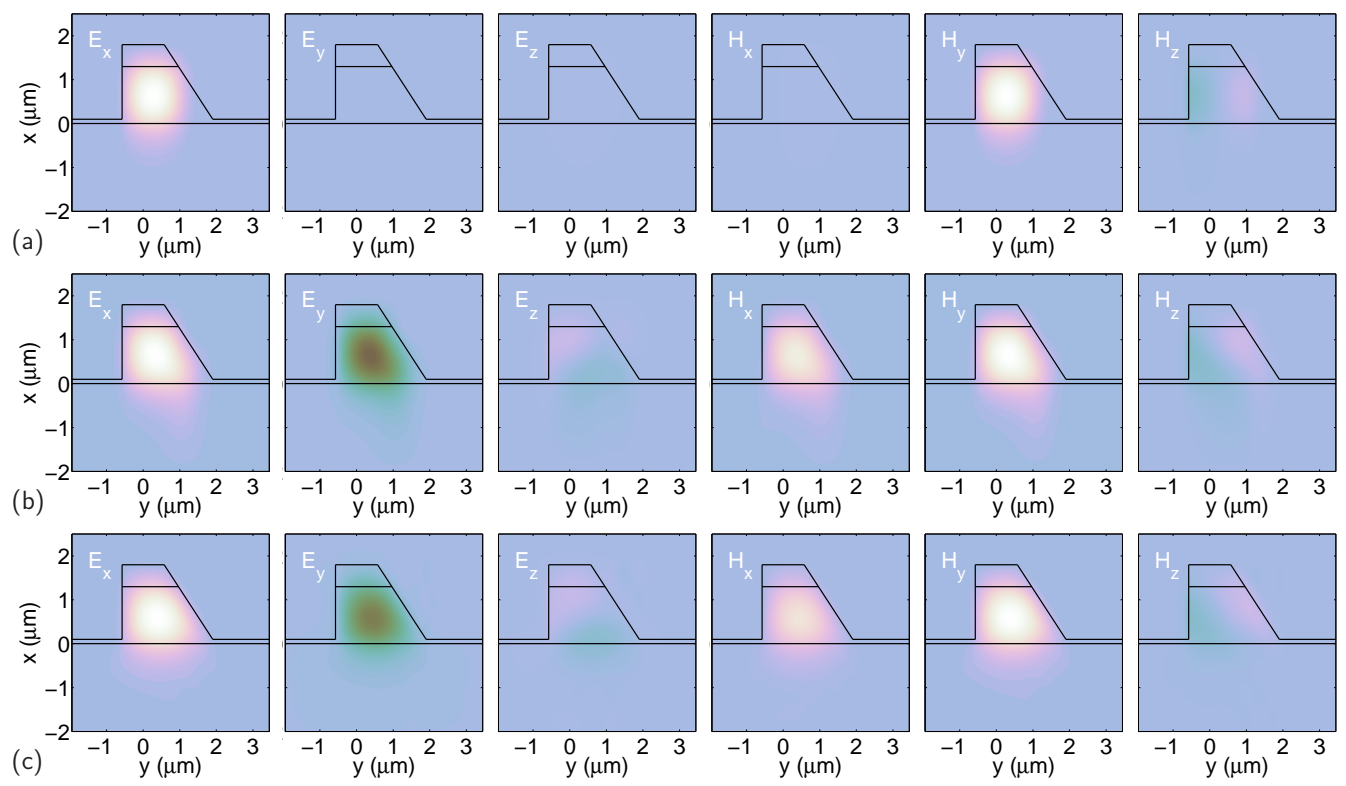

Figure 3.13: Vectorial field profile of the fundamental mode of the polariza-

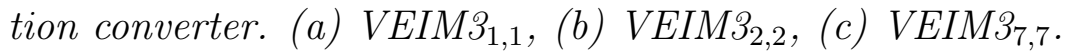

Figure 3.14 shows the convergence of the effective index of the fundamental mode of the waveguide versus the number of modes in the 3-component expansion VEIM $3_{a, b}$ (3.4.15), with $a$ and $b$ being numbers of TE and TM slab modes from the central $(y \in(0,1.15) \mu m)$ slab. It also shows the convergence of the commercial FMM mode solver [10], in which the structure is subdivided into 50 slices. Remarkably, starting from just 2 TE and TM modes in the 3-component expansion (3.4.15) VEIM $3_{2,2}$, the effective index is stable and close to the converged value of the FMM solver; 320 modes in the FMM solver lead to an effective index of 3.2225 , while with just 7 TE and TM modes the current method predicts already an effective index of 3.2223. The field profiles also converge rapidly; Figure 3.13 shows the 
vectorial fields for (a) one (VEIM3 $\left.3_{1,1}\right)$, (b) two $\left(\right.$ VEIM $\left.3_{2,2}\right)$, and (c) seven $\left(\mathrm{VEIM}_{7,7}\right)$ TE and TM modes in the expansion (3.4.15).

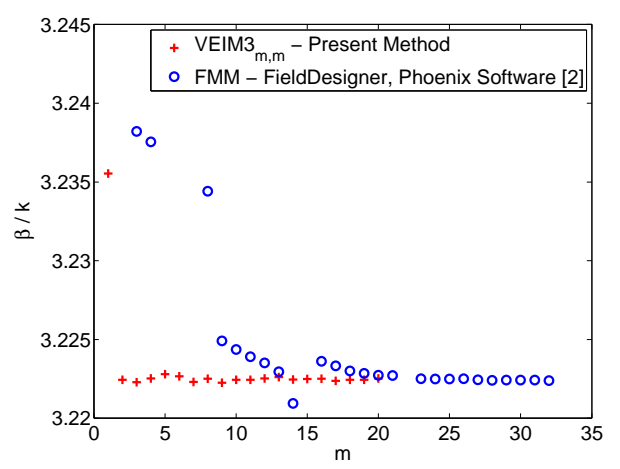

Figure 3.14: Convergence of the effective index of the fundamental mode of the polarization converter.

\subsubsection{Indiffused waveguide}

To show the flexibility of the present method we apply it to a diffused waveguide [85] with a refractive index distribution given by

$$
n^{2}(x, y)= \begin{cases}n_{s}^{2}+n_{s}^{2}\left(1.05^{2}-1\right) \exp \left(-x^{2} / 16\right) \exp \left(-y^{2} / 4\right), & \text { if } x>0 \\ n_{c}^{2}, \quad \text { if } x<0,\end{cases}
$$

with $n_{s}^{2}=2.1, n_{c}^{2}=1.0$ and $\lambda=1.3 \mu \mathrm{m}$. Similar to the slanted sidewall waveguide described above, the finite element implementation of the presented method is the more suitable, since it takes into account the nonuniform distribution in the $y$-direction of the refractive index automatically. Vertically, the structure is subdivided into 7 layers; horizontally, 20 finite elements are used. The computational window used in the calculations is defined as $(x, y) \in[-1,8] \times[-6,6] \mu m^{2}$.

Figure 3.15 shows the convergence of the effective index of the fundamental mode of the indiffused waveguide versus the number of modes in the 3- $\left(\mathrm{VEIM} 3_{a, b}\right)$ and 5-component (VEIM $\left.5_{a, b}\right)$ approximations, with $a$ and $b$ being numbers of TE and TM slab modes of the central $(y=0 \mu m)$ slab. The results are compared to the rigorous Finite Difference simulation (with $129 \times 129$ grid points) [10]. Since the fundamental mode is strongly polarized, the semi-vectorial approximation appears to converge much faster.

On Figure 3.16 field profiles of the dominant electric component $E_{y}$ of the fundamental TE mode are shown. The effective index $N_{\text {eff }}=\beta \lambda / 2 \pi$ of the 

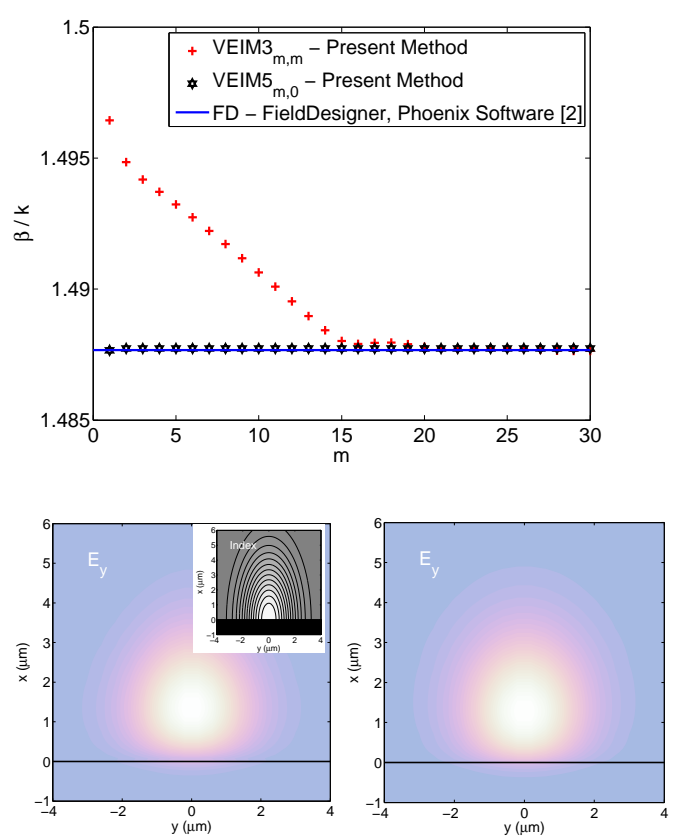

Figure 3.15: Convergence of the effective index of the fundamental mode of the diffused waveguide.

fundamental mode on the left picture is 1.4965 and on the right -1.48802 , which compares well with the Finite Difference simulation - 1.48797.

\subsection{Concluding remarks}

A variational method for the fully vectorial analysis of arbitrary isotropic dielectric waveguides was developed. Similar to the scalar approach of Chapter 2 this method gives rather accurate estimates of the propagation constants, sometimes even with only a few terms in the expansion.

When applying the present method with only one slab mode in the expansion of the modal field of the complete waveguide, this mode is transformed in all different slices to fit the true solution there the best. Together with the shape transformation, the effective index of this mode is uniquely transformed. Additionally, the expression for the transformed propagation constant is quite simple and is certainly not more complicated, than the calculation of a slab mode. In this way the present procedure turns out to be a simple and still a more rigorous way to obtain a first intuitive guess for the propagation constant and field profile, than the standard Effective Index Method.

It turns out that in case a TE mode is used in the expansion, the reduced 
equation appears to be a TM mode equation. At the same time when a TM mode is used, the reduced equation appears to be neither TE, nor TM mode equation, but something in between, with the effective refractive indices appearing both under the derivative sign and in the right part of the equation.

While in the Film Mode Matching method, rotated modes of each slice are used to locally expand the field, VEIM uses only one set of modes everywhere. We showed that in the reference slice, where the 1D modes are calculated, VEIM predicts exactly the same rotations as the Film Mode Matching method uses. In the reference slice the total field profile is a superposition of these rotated 1D TE and TM modes; in other slices, however, the components of all the 1D modes mix.

Of course the question remains - would some other combination of slab mode components lead to faster convergence? For example, one could imagine that in a certain case a superposition of e.g. explicitly selected profiles of specific slices would lead to similar results as if one would take functions related to more, let's say, five but consecutive modes - from the fundamental to the fourth order. However, adding field profiles from different slices may lead to a (near) linear dependency of functions $X$, and result in non-unique functions $Y$. Obviously, the safe choice is to use in the approximation of a component of the total field only profiles from a single slice. Nevertheless, when only a few modal components are used, it may, as our calculations show, be beneficial to use one or two modes from other slice(s).

Similar ideas can be applied to optical scattering problems in 2D and $3 \mathrm{D}$, as will be shown in Chapters 4 and 5 . 


\section{D scattering problems}

This chapter describes a simulation method for 2D frequency domain scattering problems in photonics. The technique reduces the spatial dimensionality of the problem by means of global, continuous mode expansion combined with a variational formalism; the resulting equations are solved using a finite element method. Transparent influx boundary conditions and perfectly matched layers are employed at the computational window boundaries. Numerical examples validate the method.

\subsection{Introduction}

Photonic integrated circuit design relies in general heavily on computational tools. Simulations are, on the one hand, employed for rough and approximate, but quick and efficient assessment of a configuration. On the other hand, the verification and fine-tuning of a design requires rigorous, accurate calculations, that typically demand a much higher computational effort. Among the multitude of existing approaches to frequency domain scattering problems in photonics we will briefly highlight two methods that are typically used for the aforementioned purposes.

Although "the" Effective Index Method (EIM) is mostly being formulated for the calculation of waveguide modes [15, 107, 101, 2, 64, 17, 71, $28,84]$, optical scattering problems can just as well be treated in terms of effective indices [104, 79, 109, 86, 36]. Replacing, more or less heuristically, the original 2D Helmholtz problem by a scalar equation for 1D fre- 
quency domain wave propagation through an effective structure, one obtains a very approximate solution at very low computational cost. In contrast, Bidirectional Eigenmode Propagation (BEP, also called Eigenmode Expansion Method or EME) schemes aim at accurate, converged solutions $[96,108,62,7,61,9]$. Meant for structures with piecewise constant permittivity, the optical electromagnetic field is expanded locally into sets of $1 \mathrm{D}$ slab modes. Bidirectional mode overlaps then connect the local expansions across interfaces.

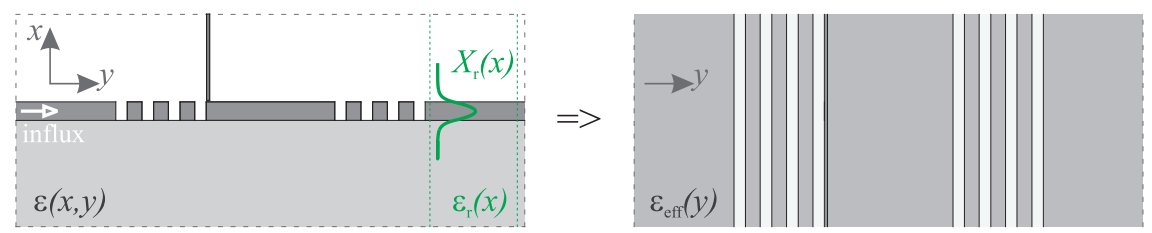

Figure 4.1: 2-to-1D dimensionality reduction, the defect grating structure with a probe tip from section 4.3.2. Using a global mode expansion together with variational techniques, the original problem in two spatial dimensions (left) is reduced to an effective system of differential equations in one spatial variable (right).

The current method is conceptually similar to what was applied in the context of scalar (Chapter 4) and vectorial (Chapter 5) mode solvers and employs a global mode expansion. A given structure is enclosed by a computational window (CW) (Figure 4.1, box), with metallic upper and lower boundaries that are equipped with Perfectly Matched Layers (PMLs) [13]. Then slab modes of one or more suitable vertical cross-section(s) are calculated (functions of $x$ in Figure 4.1), and one assumes that a superposition of these modes gives a reasonable approximation to the true field profile everywhere on the horizontal axis $y$. A variational restriction procedure [31] then allows to extract a system of second order differential equations for the unknown, now only $y$-dependent, coefficient functions. To find a numerical solution to this system we use a Finite Element Method (FEM) [18]. Transparent Influx Boundary Conditions (TIBCs) [68, 31, 89, 93] are implemented at the left and right boundaries of the computational domain. These permit to prescribe the given influx, while radiation from within the CW can freely pass through the left and right boundary; at the top and bottom of the CW, the PMLs make sure that scattered light is not reflected back from the boundaries. 
Note that the present scheme uses an expansion basis quite similar to a conventional BEP approach. Our expansions, however, are global; continuity requirements at vertical interfaces are "automatically" satisfied. In combination with a suitable selection of basis modes, the variational procedure generates reasonable approximations already with relatively few expansion terms. As an extreme case, expansions with only one vertical mode can be viewed as a variant of an EIM. Contrary to the "standard" formulations, the present approach allows to determine uniquely the effective indices, even if a local slab does not support any guided modes. While in this chapter we will only touch this particular case, more detailed remarks can be found in Ref. [36].

Apart from the EIM and BEP versions cited before, relations with the bidirectional mode propagation algorithm of Ref. [105] can be observed. Global expansions are employed there as well; the fields are expanded into Fourier series. While that standard basis allows for a highly efficient handling, apart from the special case of a locally homogeneous region the basis modes are nowhere exact solutions, such that also there a rather large number of terms might be required to generate acceptable approximations (so far, however, we did not carry out a direct comparison of efficiencies).

A brief preliminary account on our method has been given in Ref. [42]. Section 4.2 describes the theoretical background in detail. We restrict the formulation to TE polarization. In principle, the formalism for TM polarized fields can be established, similar to the scalar analysis of guided TM modes in Chapter 4. Examples and explicit equations for the situation with single mode expansions are included in Ref. [36]. In Sections 4.2.2 and 4.2.3 we pay some attention to the PML related particularities of the expansion basis, and to aspects of the numerical solution. Examples for a series of $2 \mathrm{D}$ configurations show the validity of the method in Section 4.3 .

\subsection{D scattering problems}

The propagation of TE-polarized monochromatic light with vacuum wavelength $\lambda$ and wavenumber $k=2 \pi / \lambda$ through a dielectric structure, defined by the permittivity distribution $\varepsilon(x, y)$, is governed by the 2D Helmholtz equation for the $z$-component of the electric field:

$$
\Delta E_{z}(x, y)+k^{2} \varepsilon(x, y) E_{z}(x, y)=0 .
$$


Solutions of Eq. (4.2.1) can also be formally found [30] as critical points of the functional [101]

$$
\mathcal{F}\left(E_{z}\right)=\int\left[\left(\nabla E_{z}(x, y)\right)^{2}-k^{2} \varepsilon(x, y) E_{z}^{2}(x, y)\right] d x d y .
$$

In the following we will use the variational formulation only. While Eqs. (4.2.1, 4.2.2) cover in principle the entire $x$-y-plane, practical computations have to be confined to a computational window. Then suitable boundary conditions need to be implemented, that allow any given influx to be prescribed and radiation to freely leave the computational window.

\subsubsection{Dimensionality reduction}

We assume that the true field profile can be reasonably approximated at every point on the horizontal $y$-axis by a superposition of $1 \mathrm{D}$ vertical slab modes. Typically these are modes that are supported by (one or more) vertical cross-sections. Note that each mode can, in principle, be taken from a different reference slab waveguide, although in practice, many (or all) may come from the same one. Then the principal field component $E_{z}$ is expanded as

$$
E_{z}(x, y)=\sum_{j=1}^{m} X_{j}(x) \cdot Y_{j}(y),
$$

where $X_{j}$ is the principal component of a TE polarized mode from a reference slab waveguide, and $Y_{j}$ is an unknown coefficient function which determines the amplitude of this mode at every position $y$.

By restricting the functional (4.2.2) to the set of functions in the form (4.2.3), and taking variations with respect to all unknowns $Y_{j}$, following a similar procedure as in Chapter 2, we find the following system of second order differential equations for the functions $Y_{j}$ :

$$
\mathbf{F Y}^{\prime \prime}(y)+\mathbf{M}(y) \mathbf{Y}(y)=0 .
$$

Here $\mathbf{Y}$ is a vector function formed of all functions $Y_{j}$, and $\mathbf{F}$ and $\mathbf{M}$ are matrices of dimension $m \times m$ with elements that are given by overlap integrals involving the modes $X_{j}$, their derivatives, and the permittivity $\varepsilon(x, y)$ :

$$
F_{g, h}=\int X_{g}(x) X_{h}(x) d x
$$




$$
M_{g, h}(y)=\int\left[k^{2} \varepsilon(x, y) X_{g}(x) X_{h}(x)-X_{g}^{\prime}(x) X_{h}^{\prime}(x)\right] d x .
$$

Beyond (4.2.4), for a structure divided into slices, the stationarity of (4.2.2) amounts to interface conditions of continuity of

$$
\mathbf{Y}(y) \text { and } \mathbf{Y}^{\prime}(y)
$$

Thus, through the approximation (4.2.3), the problem of finding the function $E_{z}(x, y): \mathbb{R}^{2} \rightarrow \mathbb{C}$ has been reduced to the task of identifying a vector function $\mathbf{Y}(y): \mathbb{R} \rightarrow \mathbb{C}^{m}$.

\subsubsection{Basis modes $X_{j}$ defined using Perfectly Matched Layers}

Before we proceed to the actual solution procedure for the problem (4.2.4) in section 4.2.3, we will introduce some short remarks on the the basis modes together with the implementation of transparency for the upper and lower computational window boundaries.

\section{Perfectly matched layers}

If one would use only Dirichlet boundary conditions at the top and bottom window boundaries (Figure 4.1), light that is scattered from the structure and reaches these boundaries would be completely reflected back into the window. In order to model an open domain, first along the $x$-axis, we employ the well-known Perfectly Matched Layers (PMLs) [3, 14, 4]. Although there are other viewpoints on PMLs, we prefer to see them in the following way: In these artificial layers, the coordinate is 'stretched' into the complex plane, providing - at least analytically - absorption of the light without causing reflections at the interior-PML boundary. We will use PMLs that act as absorbers along the $x$ direction, where inside the PML the coordinate $x$ is transformed as

$$
x \rightarrow x-\mathrm{i} \int_{0}^{x} \sigma(\tilde{x}) d \tilde{x}
$$

meaning that $d x$ and $\frac{\partial}{\partial x}$ are transformed in the following way:

$$
d x \rightarrow(1-\mathrm{i} \sigma(x)) d x, \quad \frac{\partial}{\partial x} \rightarrow \frac{1}{1-\mathrm{i} \sigma(x)} \frac{\partial}{\partial x} .
$$


Let's consider how a plane wave behaves in a PML. Under the given transformations, a wave that would propagate with $x$-dependence $\exp \left(-\mathrm{i} k_{x} x\right)$ in non-PML media will instead behave as $\exp \left(-\mathrm{i} k_{x}\left(x-\mathrm{i} \int_{0}^{x} \sigma(\tilde{x}) d \tilde{x}\right)\right)$ - i.e. if $\sigma$ is positive, it is attenuated in the positive $x$ direction. Similarly, a wave with $x$-dependence $\exp \left(\mathrm{i} k_{x} x\right)$ in non-PML media, inside the PML is attenuated in the negative $x$-direction. The consequence of this is that if a PML is placed just before a totally reflecting boundary, waves impinging on the boundary are first attenuated while traversing the PML toward the boundary, reflected, and subsequently attenuated once more. This double attenuation makes PMLs very powerful as absorbing boundary conditions in simulations of Maxwell's equations.

The parameter $\sigma(x)$, sometimes called the PML strength, equals zero outside the PML. Inside the PML, we choose it to increase linearly from zero at the interior-PML interface, to a maximum value $\sigma_{\max }$ at the outer boundary of the domain. The reason for a slow increase in $\sigma(x)$ is that even though the PML is analytically reflectionless, numerically a quick spatial variation of the PML strength can cause some reflections.

\section{D slab modes with PMLs}

In the presence of PMLs that absorb in the $x$-direction, the modified Helmholtz equation (4.2.1) is

$$
\left(\partial_{y y}+\frac{1}{1-\mathrm{i} \sigma(x)} \partial_{x} \frac{1}{1-\mathrm{i} \sigma(x)} \partial_{x}+k^{2} \varepsilon(x, y)\right) E_{z}(x, y)=0
$$

where $\sigma(x)$ is as described at the end of the previous subsection.

In case the refractive index does not depend on $y, \varepsilon(x, y)=\varepsilon_{\mathrm{r}}(x)$, Eq. (4.2.10) permits modal solutions of the form $E_{z}(x, y)=X(x) \mathrm{e}^{-\mathrm{i} \beta y}$, where the complex mode profile $X$ satisfies the equation

$$
\left(\frac{1}{1-\mathrm{i} \sigma(x)} \partial_{x} \frac{1}{1-\mathrm{i} \sigma(x)} \partial_{x}+\left(k^{2} \varepsilon_{\mathrm{r}}(x)-\beta^{2}\right)\right) X(x)=0,
$$

for a complex propagation constant $\beta$.

If we consider a domain $x \in[a, b]$ with zero Dirichlet boundary conditions $X(a)=0$ and $X(b)=0$, solutions $X_{j}$ of the eigenvalue equation (4.2.11) are orthogonal in the following dot product [25]:

$$
(u(x), v(x))=\int_{a}^{b} u(x) v(x)(1-\mathrm{i} \sigma(x)) d x .
$$


To solve Eq. (4.2.11), we employ a standard finite element (FE) method, using a nonuniform 1D grid generated by a commercial package [18] with linear basis functions and constant coefficients in each element. The finite element scheme results in a generalized eigenvalue problem, the solutions of which approximate the eigenfunctions $X_{j}$ and eigenvalues $\beta_{j}^{2}$ of Eq. (4.2.11). Using a FE scheme has the additional advantage that the overlap integrals (4.2.5), (4.2.6) become trivial to calculate.

\section{Use of PMLs in the expansion}

In order to use PMLs in the theory described in the sections 4.2 and 4.2.1, what is needed is to consistently apply the transformations given in Eq. (4.2.9) - which formally affect Eq. (4.2.1) and the functional (4.2.2), and consequently also the calculation of the overlap integrals in (4.2.5) and (4.2.6). Here, weighting factors as in Eq. (4.2.12) appear as the only complication required in practice.

\subsubsection{Method of solution of the reduced problem}

In general an integrated optics component can have an arbitrary permittivity distribution, e.g. tapers (Figure 4.10) or sinusoidal gratings. In section 4.2 .3 we will detail a numerical method of solution based on the Finite Element Method. Due to its general applicability and ease of implementation, we will only use this solution technique in the numerical results that will follow. At the same time for a large class of problems the system (4.2.4) could be solved semi-analytically, as we will briefly describe in section 4.2.3.

\section{Finite Element Method combined with Transparent Influx Bound- ary Conditions}

Using a Finite Element Method to solve the system of differential equations allows for more freedom in the structure than the semi-analytic method described below. For example, it can handle interfaces that do not run parallel to $x$ or $y$, like tapered waveguides.

While the system (4.2.4) is defined on the whole $\mathbb{R}$, we have to restrict our finite element computations to the finite numerical window. Since we deal with influx interacting with optical structure, it is important to have proper boundary conditions that represent the correct physical properties of the exterior of the computational domain. Boundary conditions should 
allow influx to be prescribed and reflected fields to propagate through the boundary without reflection. For this purpose we use so-called Transparent Influx Boundary Conditions [30, 68, 89, 93, 34, 50].

As a requirement we have to choose the computational window $y \in[l, r]$ in such a way that outside of it the optical structure is invariant in the $y$-direction. Then we can readily write a weak formulation for the system (4.2.4) [50, 52]:

$$
\begin{aligned}
& \int_{l}^{r} \mathbf{V}^{\top}(y)\left[\mathbf{F}^{-1} \mathbf{M}(y) \mathbf{Y}(y)+\mathbf{Y}^{\prime \prime}(y)\right] d y- \\
& -\mathbf{V}^{\top}(r)\left[\mathbf{Y}^{\prime}(r)-\mathbf{Y}_{\text {ext }}^{\prime}(r)\right]+\mathbf{V}^{\top}(l)\left[\mathbf{Y}^{\prime}(l)-\mathbf{Y}_{\text {ext }}^{\prime}(l)\right]=0,(4.2 .13)
\end{aligned}
$$

where $\mathbf{V}$ is a continuous test vector-function defined on $y \in[l, r] . \quad \mathbf{Y}_{\text {ext }}$ represents a - still to be established - solution to the system (4.2.4) outside the computational interval. Dashes indicate $y$ derivatives, the symbol ${ }^{\top}$ denotes the transpose. Note that $\mathbf{Y}$ is meant here to be defined exclusively on the interior interval $[l, r]$, and $\mathbf{Y}_{\text {ext }}$ - on the exterior regions $(-\infty, l] \cup$ $[r, \infty)$. Where necessary, derivatives are to be taken as one-sided limits.

By requiring that Eq. (4.2.13) is satisfied for arbitrary $\mathbf{V}$, one recovers the system (4.2.4) for $y \in[l, r]$, together with continuity $\mathbf{Y}_{\text {ext }}(l)=\mathbf{Y}(l)$ and $\mathbf{Y}(r)=\mathbf{Y}_{\text {ext }}(r)$ of the functions (essential boundary conditions); and continuity $\mathbf{Y}_{\text {ext }}^{\prime}(l)=\mathbf{Y}^{\prime}(l)$ and $\mathbf{Y}^{\prime}(r)=\mathbf{Y}_{\text {ext }}^{\prime}(r)$ of the derivatives across the boundary (natural boundary conditions). Integrating by parts the term with the second derivative transforms Eq. (4.2.13) to a standard weak form, with simpler boundary terms:

$$
\begin{aligned}
& \int_{l}^{r}\left[\mathbf{V}^{\top}(y) \mathbf{F}^{-1} \mathbf{M}(y) \mathbf{Y}(y)-\left(\mathbf{V}^{\top}(y)\right)^{\prime} \mathbf{Y}^{\prime}(y)\right] d y+ \\
& +\mathbf{V}^{\top}(r) \mathbf{Y}_{\mathrm{ext}}^{\prime}(r)-\mathbf{V}^{\top}(l) \mathbf{Y}_{\mathrm{ext}}^{\prime}(l)=0 .
\end{aligned}
$$

Now, the invariance along $y$ of the permittivity distribution outside the computational domain makes it possible to construct the exterior solution $\mathbf{Y}_{\text {ext }}$ [93]. Since $\mathbf{M}$ is constant for $y<l$, a particular solution of Eq. (4.2.4) in that region is

$$
\mathbf{Y}_{\text {ext }}(y)=a \mathrm{e}^{\mathrm{i} \lambda y} \mathbf{p} \text { for } y<l,
$$

where $a$ is an arbitrary amplitude, and $\lambda$ and $\mathbf{p}$ satisfy the eigenvalue problem

$$
\mathbf{M p}=\lambda^{2} \mathbf{F} \mathbf{p}
$$


To proceed further we look a bit closer at the properties of the matrices entering this eigenvalue problem. Alternatively to the expression (4.2.6), we can consider

$$
M_{g, h}(y)=\beta_{h}^{2} F_{g, h}+k^{2} \int\left[\varepsilon(x, y)-\varepsilon_{h}(x)\right] X_{g}(x) X_{h}(x) d x,
$$

derived from (4.2.6) using once integration by parts and the differential equation for the mode profile $X_{h}$. If it happens that the waveguide with the permittivity $\varepsilon_{h}(x)$ and which supports the mode profile $X_{h}$, coincides with the one that extends into the left exterior $y<l$, then to the left of the computational window $\varepsilon(x, y)=\varepsilon_{h}(x)$, and the second term of (4.2.17) vanishes. Thus column $h$ of the matrix $\mathbf{M}$ is equal to column $h$ of the matrix $\mathbf{F}$ times the square of $\beta_{h}$, the propagation constant of mode $h$. Hence the eigenvalue problem (4.2.16) permits a solution pair: eigenvalue $\lambda^{2}=\beta_{h}^{2}$ and corresponding unit eigenvector $\mathbf{p}$, with an entry 1 at position $h$. This means that in the left exterior this particular mode $X_{h}$ decouples from all other modes that enter the expansion (4.2.3) and contributes to the solution for $y<l$ as

$$
E_{z}(x, y)=\left(a_{1} \mathrm{e}^{\mathrm{i} \beta_{h} y}+a_{2} \mathrm{e}^{-\mathrm{i} \beta_{h} y}\right) X_{h}(x)+\ldots,
$$

with some coefficients $a_{1}$ and $a_{2}$. It implies that while all the other modes can be coupled through the non-unit eigenvectors $\mathbf{p}$, those that originally were computed for the same waveguide as the one in the left exterior, are not modified by the expansion (4.2.3). This property then makes it trivial to prescribe the influx through the computational boundary in terms of the modes of the incoming waveguide. Let us say we want to excite the optical structure by the mode $X_{h}$ with amplitude $q$, which implies that

$$
\mathbf{Y}_{\text {ext }}(y)=\sum_{s=1, \ldots, h, \ldots, m} a_{s} \mathrm{e}^{\mathrm{i} \lambda_{s} y} \mathbf{p}_{s}+q \mathrm{e}^{-\mathrm{i} \lambda_{h} y} \mathbf{p}_{h} \text { for } y<l
$$

with yet unknown coefficients $a_{s}$. We can rewrite this equation in matrix form by defining a to be a column-vector of all coefficients $a_{s} ; \mathbf{E}(y)-$ a diagonal matrix with entries $\mathrm{e}^{\mathrm{i} \lambda_{s} y}$; and $\mathbf{P}-$ a matrix, composed by the vectors $\mathbf{p}_{s}$ as columns:

$$
\mathbf{Y}_{\text {ext }}(y)=\mathbf{P E}(y) \mathbf{a}+q \mathrm{e}^{-\mathrm{i} \lambda_{h} y} \mathbf{p}_{h}
$$


Next, since the continuity conditions (4.2.7) require that $\mathbf{Y}_{\text {ext }}(y)=\mathbf{Y}(y)$ at $y=l$, we can express these coefficients in terms of the interior solution $\mathbf{Y}$ at the boundary:

$$
\mathbf{a}=\mathbf{E}^{-1}(l) \mathbf{P}^{-1}\left[\mathbf{Y}(l)-q \mathrm{e}^{-\mathrm{i} \lambda_{h} l} \mathbf{p}_{h}\right]
$$

These coefficients then permit to express also the derivative of the exterior solution $\mathbf{Y}_{\text {ext }}^{\prime}(l)$ in terms of the interior solution $\mathbf{Y}(l)$ at the boundary. Differentiating Eq. (4.2.19) gives

$$
\begin{aligned}
\mathbf{Y}_{\text {ext }}^{\prime}(y) & =\sum_{s=1, \ldots, h, \ldots, m} a_{s} \mathrm{i} \lambda_{s} \mathrm{e}^{\mathrm{i} \lambda_{s} y} \mathbf{p}_{s}-\mathrm{i} q \lambda_{h} \mathrm{e}^{-\mathrm{i} \lambda_{h} y} \mathbf{p}_{h}= \\
& =\mathrm{i} \mathbf{P} \mathbf{\Lambda} \mathbf{E}(y) \mathbf{a}-\mathrm{i} q \lambda_{h} \mathrm{e}^{-\mathrm{i} \lambda_{h} y} \mathbf{p}_{h}= \\
& =\mathrm{i} \mathbf{P} \boldsymbol{\Lambda} \mathbf{E}(y)\left[\mathbf{E}^{-1}(l) \mathbf{P}^{-1}\left[\mathbf{Y}(l)-q \mathrm{e}^{\left.-\mathrm{i} \lambda_{h} l_{\mathbf{p}_{h}}\right]}\right]-\mathrm{i} q \lambda_{h} \mathrm{e}^{-\mathrm{i} \lambda_{h} y} \mathbf{p}_{h}\right.
\end{aligned}
$$

where $\boldsymbol{\Lambda}$ is a diagonal matrix with diagonal entries $\lambda_{s}$. So at the boundary $y=l$ we have after some simplifications

$$
\mathbf{Y}_{\mathrm{ext}}^{\prime}(l)=\mathrm{i} \mathbf{P} \boldsymbol{\Lambda} \mathbf{P}^{-1} \mathbf{Y}(l)-\mathrm{i} 2 q \lambda_{h} \mathrm{e}^{-\mathrm{i} \lambda_{h} l} \mathbf{p}_{h}
$$

Inserting this into the weak formulation (4.2.14), together with an analogous expression for $\mathbf{Y}_{\text {ext }}^{\prime}(r)$ at the right boundary, we are finally able to restrict the weak formulation to the computational domain only, with all information about the exterior hidden inside the matrices $\mathbf{P}$ and $\boldsymbol{\Lambda}$.

\section{Semi-analytic solution method}

Due to common techniques of fabrication, many components of integrated optical devices have a piecewise constant rectangular permittivity distribution, e.g. the rectangular grating of Figure 4.2. This property of the device geometry results in a piecewise constant matrix $\mathbf{M}(y)$. Therefore the general solution of Eq. (4.2.4) can be written out on every interval where the permittivity is invariant in the $y$-direction. These local solutions then can be matched across the interfaces according to the interface conditions (4.2.7). We refer to Chapter 2 and Ref. [35] for more detailed remarks on such approaches. 


\subsubsection{Relation with the Effective Index Method}

Regarding the relation between the present approach and "conventional" effective index methods, it is instructive to look at the expansion (4.2.3) with only a single term, $E_{z}(x, y)=X(x) Y(y)$. Here $X$ will typically be the fundamental guided mode of a suitable reference slab (Figure 4.1), that is likely to represent a major part of the actual physical field. For the specific case of guided modes we can use an unbounded domain in $x$ without PMLs. Let $\varepsilon_{\mathrm{r}}(x)$ be the permittivity of that reference slab, and $\beta_{\mathrm{r}}$ be the propagation constant, such that $X$ satisfies the slab mode equation

$$
X^{\prime \prime}(x)+k^{2} \varepsilon_{\mathrm{r}}(x) X(x)=\beta_{\mathrm{r}}^{2} X(x) .
$$

Then the system (4.2.4) reduces to the following EIM-like equation for the function $Y$ :

$$
Y^{\prime \prime}(y)+k^{2} \varepsilon_{\mathrm{eff}}(y) Y(y)=0,
$$

with the effective permittivity

$$
\varepsilon_{\mathrm{eff}}(y)=\frac{\beta_{\mathrm{r}}^{2}}{k^{2}}+\frac{\int\left(\varepsilon(x, y)-\varepsilon_{\mathrm{r}}(x)\right) X^{2}(x) d x}{\int X^{2}(x) d x} .
$$

Similarly to the standard EIM, in the reference slice, where $\varepsilon(x, y)=\varepsilon_{\mathrm{r}}(x)$, the effective permittivity is that of the mode $X$. However, in other slices it is modified by the difference between the local permittivity and that of the reference slice, weighted by the local intensity of the mode profile. So, contrary to the EIM, even in slices where no guided modes exist, an effective index can still be uniquely defined. Note that it may turn out that effective permittivity found from (4.2.28) is negative. We refer to [36] for a more detailed elaboration of this viewpoint, including a series of numerical examples.

\subsection{Numerical results}

The presented theory is implemented with the aid of the COMSOL Multiphysics package [18] for grid generation and for setting up the finite element equations in the $y$-direction; the slab modes are calculated by means of an in-house developed finite element code. This section shows and discusses results on three structures: A waveguide Bragg grating, a perturbed defect cavity in a grating, and a vertical taper. The acronym VEIM (variational effective index method) shall be used to indicate results of our approach. 


\subsubsection{Waveguide Bragg grating}

The waveguide Bragg grating of Figure 4.2 served as a benchmark structure to compare independently developed 2D numerical codes in the COST 268 modelling task [106]. The grating is formed by etching 20 rectangular grooves into a $\mathrm{Si}_{3} \mathrm{~N}_{4} / \mathrm{SiO}_{2}$ slab waveguide. We are interested in the response of the waveguide grating, i.e. the relative guided wave transmission $T$ and reflection $R$, for the incoming fundamental TE mode at varying vacuum wavelengths $\lambda$.

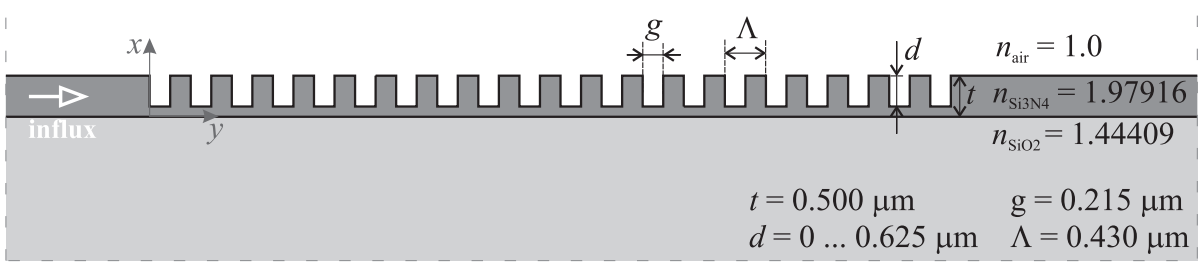

Figure 4.2: A waveguide Bragg grating, benchmark structure of Refs. [106], [6] and [33].

Slab modes are calculated on a computational window $x \in[-3,3] \mu m$ with zero Dirichlet boundaries, coated with $1 \mu m$ thick PMLs, whose strength increases linearly towards the boundaries from 0 to $\sigma_{\max }=0.8$. The expansion uses 40 slab modes of the input waveguide, which are calculated with 320 linear elements in the vertical direction. The horizontal extent $y \in[-1,9] \mu m$ of the computational window is discretized into 352 quadratic finite elements.

Figure 4.3 shows a comparison of results of the present method (VEIM) to those generated by two other modelling schemes. The curves labelled QUEP are computed using the semi-analytic Quadridirectional Eigenmode Propagation scheme from Ref. [33], and the label BEP denotes results from a Bidirectional Eigenmode Propagation method with PMLs at the boundaries from Ref. [106]. The agreement between the present method and the reference results is excellent. 


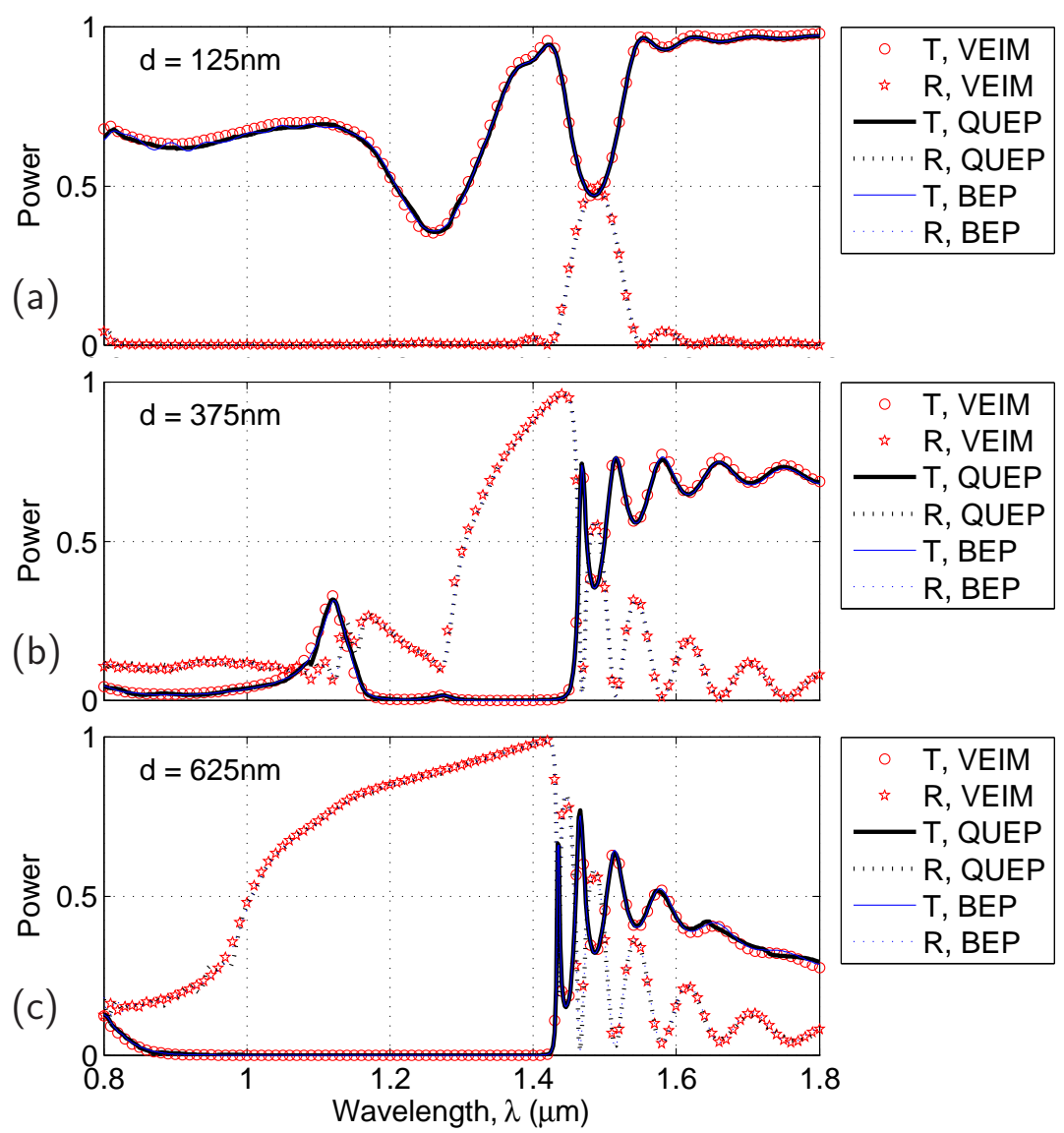

Figure 4.3: $\quad$ Spectral propagation properties, modal reflection $R$ and transmission $T$, for the grating of Figure 4.2, calculated by three modelling methods. Groove depth d: (a) $125 \mathrm{~nm}$, (b) $375 \mathrm{~nm}$ and (c) $625 \mathrm{~nm}$.

\subsubsection{Waveguide Bragg grating perturbed by a nano- sized probe}

The authors of Ref. [37] describe a grating with a defect cavity. This structure exhibits a bandgap in which the transmission is very small, with a narrow transmission peak inside it, due to the defect. The spectrum of this cavity resonance can be shifted in wavelength, while also being deformed, by the presence of a thin silicon probe that is placed on top of it. Figure 4.4 introduces the structure. For the VEIM simulations, the calculation of the slab modes is based on a discretization of the slab profile (outside the holes) 
into 736 linear finite elements; 280 nonuniformly distributed quadratic elements are used to solve the equations along the $y$-axis.

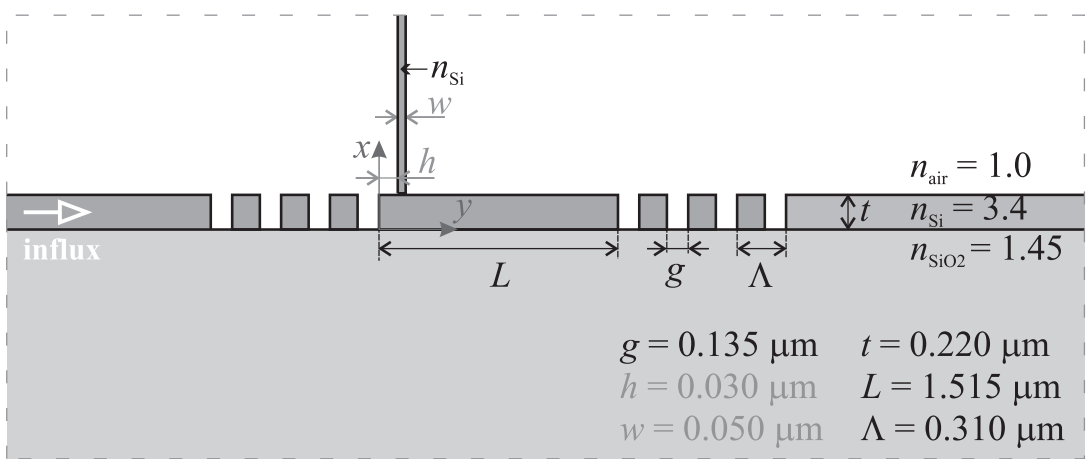

Figure 4.4: A waveguide Bragg grating with defect, perturbed by a thin vertical Si probe, from Ref. [37].
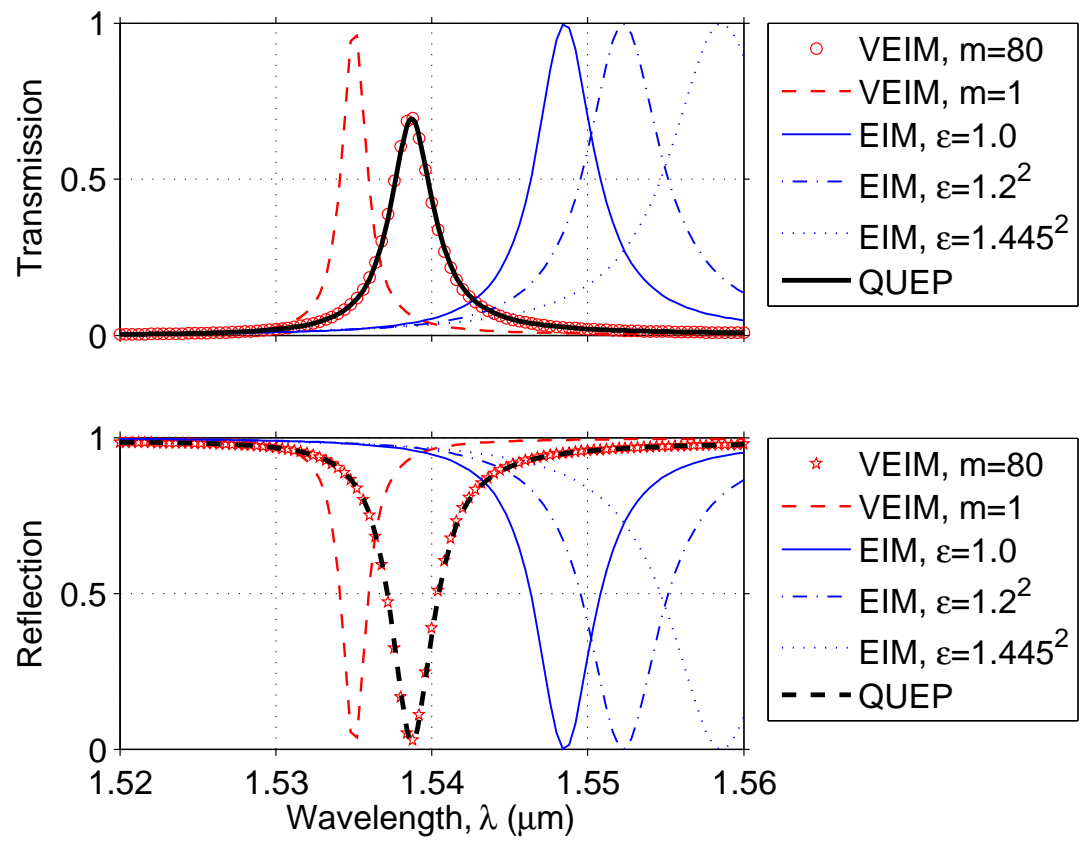

Figure 4.5: Resonant transmission through the defect grating of Figure 4.4, for different computational methods.

First we consider the grating without the probe. Since the present 
method with one mode in the expansion requires a similar computational effort as the Effective Index Method, we compare results of those two methods. As mentioned in section 4.2.4, the effective index in a hole in the structure is not uniquely defined, since no guided mode exists there. Therefore, we choose three values that seem reasonable - the highest local refractive index 1.445 (the substrate), the lowest refractive index 1.0 (air), and an in-between value of 1.2. Figure 4.5 shows the transmission and reflection spectra resulting from these choices in a standard EIM, results from our VEIM with 1 and 80 modes in the expansion, and finally, as a reference, spectra calculated using the QUEP method [33].

The VEIM, $m=80$ results virtually coincide with the reference results. Using only one mode in the expansion (VEIM, $m=1$ ) shifts the spectrum to lower wavelengths and removes the loss mechanisms from the simulations, so the peak in the spectrum is more pronounced, and the reflection and transmission add up to unity. The EIM results are likewise lossless, and their spectra are all shifted to higher wavelengths - and the spectral peaks for all reasonable values of the effective index in the hole regions are displaced from the reference results much further than the VEIM, $m=1$ results.

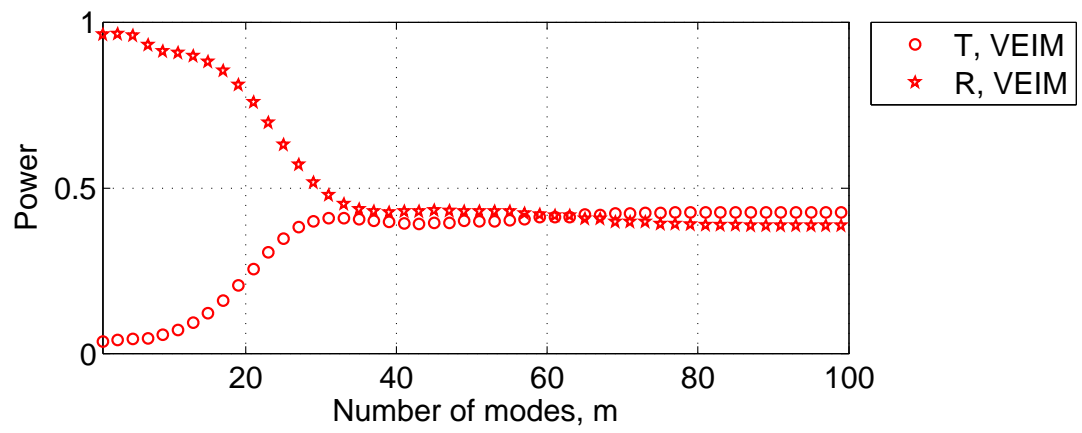

Figure 4.6: Convergence of transmission $T$ and reflection $R$ at wavelength $\lambda=1.54 \mu m$ versus the number of modes $m$ in VEIM simulations of the grating of Figure 4.4, for absent tip.

To investigate how many modes are needed to accurately simulate a structure like this grating, we perform a convergence analysis in the number of modes in the expansion. We choose a wavelength of $1.54 \mu \mathrm{m}$, which lies on the flank of the resonance; this is where the sensitivity of transmission and reflection to numerical errors is expected to be the highest. Figure 4.6 shows 
the results. Starting from 40 modes, the levels are quite stable already, and at 80 modes we may consider the calculations to be converged.

Figure 4.7 shows field plots of the grating at the resonance wavelength of $1.5387 \mu \mathrm{m}$, for VEIM expansions with one, ten and eighty modes. With one mode, there is no radiation, and the transmission is low. Using ten modes already allows for significant radiation, but the reflection and transmission values are still relatively far from the converged values.

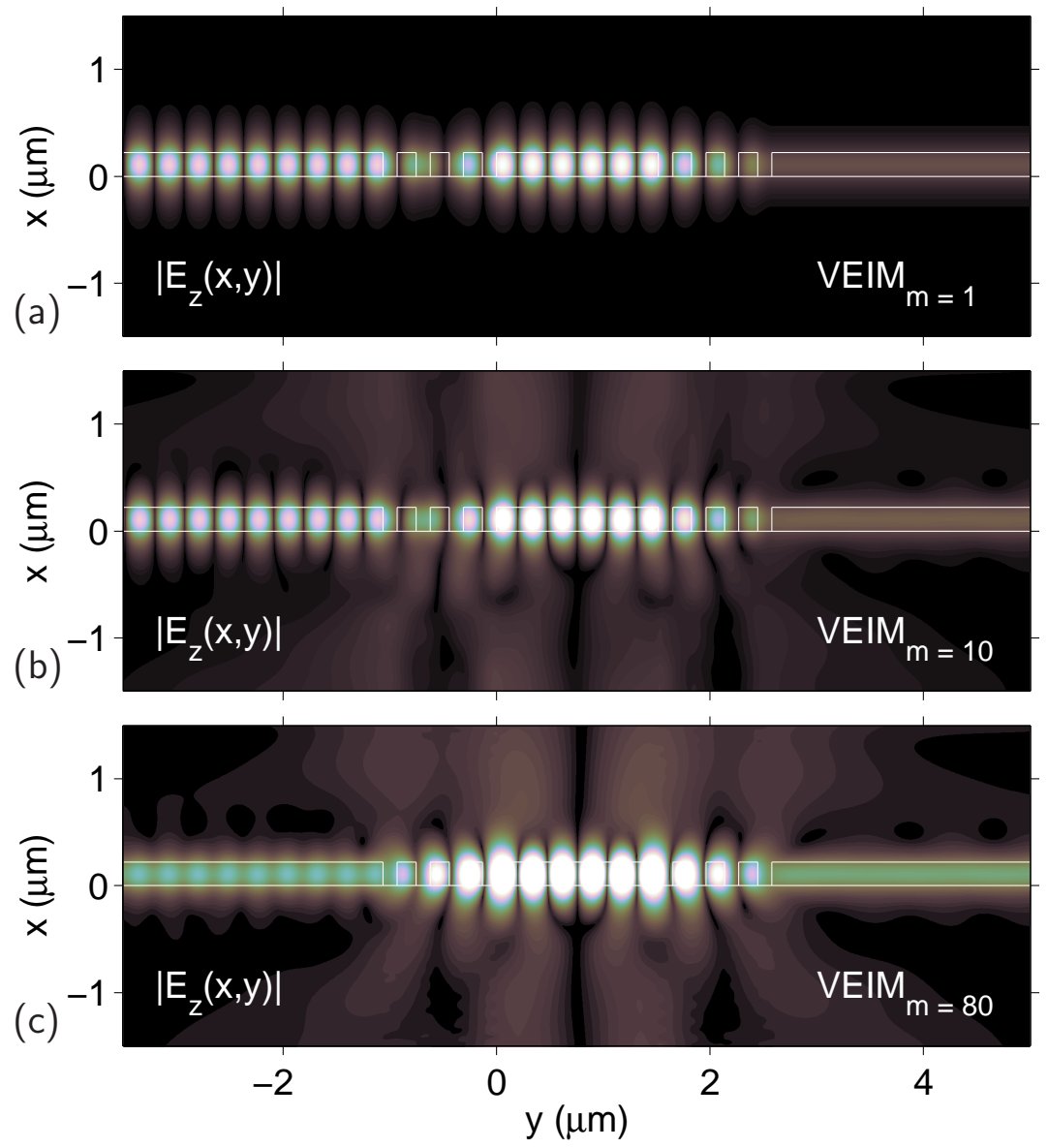

Figure 4.7: Absolute value of the field profile $E_{z}$ for the grating of Figure 4.4. without the tip, at the resonance wavelength $\lambda=1.5387 \mu \mathrm{m}$. (a) a VEIM simulation with only one mode in the expansion; (b) VEIM expansion with 10 modes; (c) the converged results for 80 modes.

When the defect cavity is perturbed with a silicon probe, the spectrum 
shifts and deforms. Figure 4.8 shows the transmission spectrum with the probe, placed near one end of the cavity, as indicated in Figure 4.4. Again, the VEIM results are very close to the reference QUEP data. Figure 4.9 shows the field for the structure with the tip at a wavelength of $1.5387 \mu \mathrm{m}$, the resonance wavelength of the unperturbed structure. The field clearly is disturbed strongly. Remarkably, one can see the fundamental mode of the silicon probe running upward - even though the present method essentially only propagates modes horizontally. The PMLs allow for radiation to leave the domain vertically.

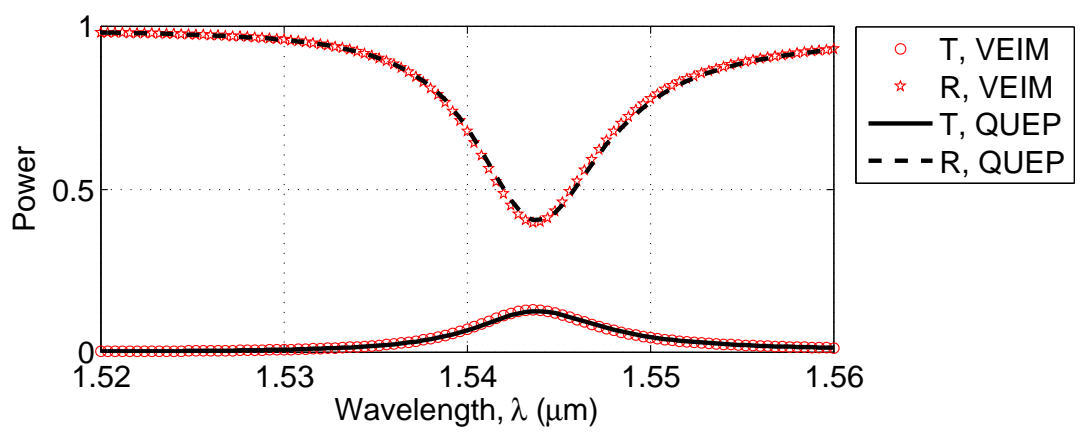

Figure 4.8: Spectral transmission $T$ and reflection $R$ through the defect grating of Figure 4.4 if the probe is present. VEIM and QUEP results are compared.

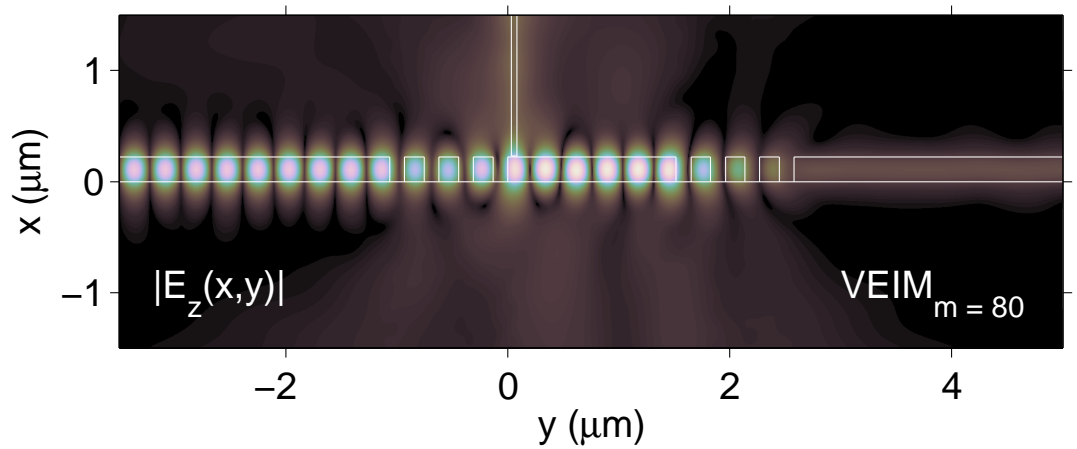

Figure 4.9: Absolute value of the field profile $E_{z}$ of the grating of Figure 4.4 with the probe on top, at the resonance wavelength $\lambda=1.5387 \mu m$ of the original (unperturbed) structure. The probe switches the device to a lowtransmission state. 


\subsubsection{Taper}

Our last example is the vertical taper of Figure 4.10. We are interested in the transmission from the fundamental mode of the left-hand waveguide to the fundamental mode of the thicker right-hand waveguide.

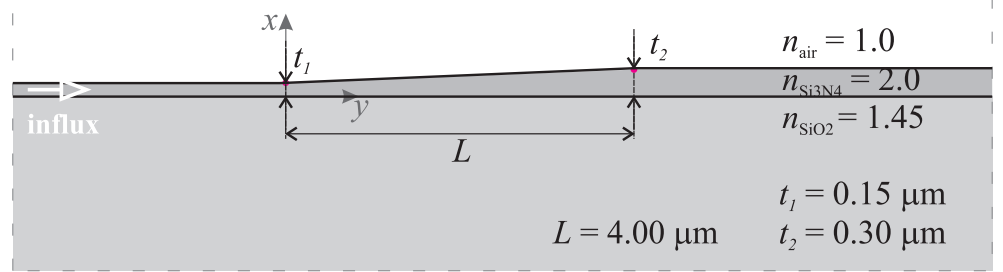

Figure 4.10: A vertical taper in $\mathrm{Si}_{3} \mathrm{~N}_{4}$ on $\mathrm{SiO}_{2}$ with air cladding, simulated at a wavelength of $1.55 \mu \mathrm{m}$. The taper is $4 \mu \mathrm{m}$ long and tapers linearly from 150 to $300 \mathrm{~nm}$ thickness.
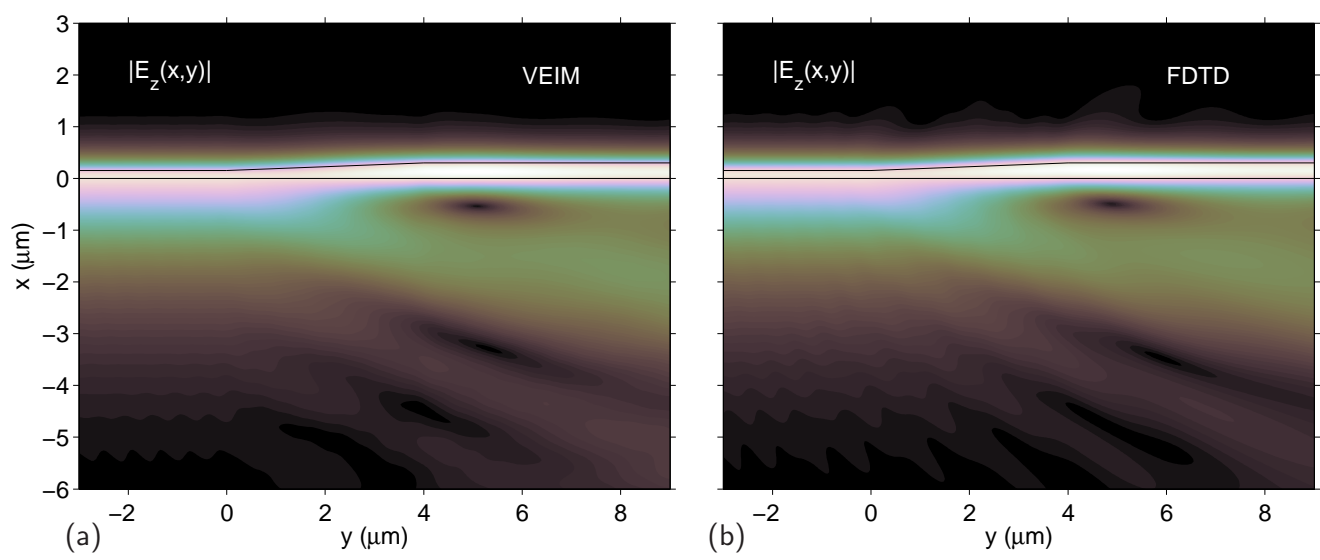

Figure 4.11: Absolute value of the field profile $E_{z}$ of the vertical taper of Figure 4.10. (a) The present method, a VEIM expansion with 10 modes from the left (thin) guide, and 1 mode from the right (thick) one. (b) FDTD reference results.

As discussed in section 4.2.3, in order to properly influx a mode, and also to analyze the outgoing power of a mode, it is beneficial to have those modes in the expansion of the vertical field. Therefore, we expand the field 
into a number of modes of the left-hand waveguide plus the fundamental mode of the right-hand guide. The maximum PML strength is set to $\sigma_{\max }=0.6$. The vertical basis modes are calculated using 368 linear finite elements; 608 quadratic elements discretize the horizontal computational window. Figure 4.11 shows the absolute value of the $E_{z}$ field for the present method, with only 10 modes from the left waveguide, together with rigorous numerical results, obtained with the Finite-Difference-Time-Domain (FDTD) solver from a commercial package [11]. Even for such a low number of modes in the expansion, the fields match remarkably well.

Results of a convergence analysis for the taper structure are given in Figure 4.12. We take one mode of the right-hand waveguide in the expansion and vary the number of modes $m$ from the left-hand waveguide. Already with 6 modes from the left-hand guide the result is well within $1 \%$ of the reference results, and with 10 modes the transmission can be said to be converged.

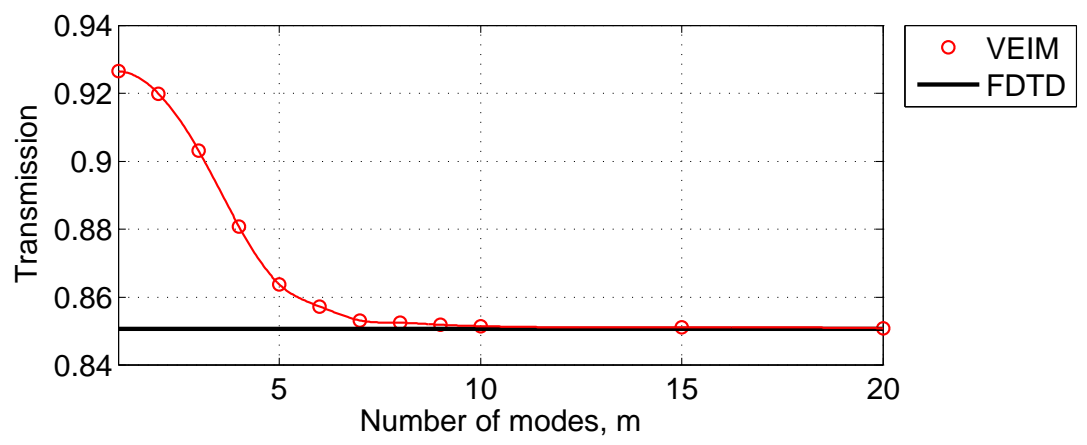

Figure 4.12: VEIM simulations of the taper of Figure 4.10, convergence: The plot shows the guided wave transmission versus the number of modes from the thin waveguide in the VEIM expansion. The thicker waveguide contributes one mode to the expansion.

\subsection{Concluding remarks}

The VEIM algorithm, as proposed in this chapter, constitutes a viable alternative to established quasi-analytical solvers for optical Helmholtz problems, so far in 2D. In particular, the method has similarities to and improves upon the popular EIM and BEP schemes: As in a standard EIM (though with less heuristics involved), rough approximations can be ob- 
tained through expansions with one or only very few terms, at very low computational cost. In an intermediate regime already quite acceptable results emerge with only a very moderate number of expansion terms, as shown in some of our examples. VEIM calculations with a larger number of basis modes yield converged results, just as the established BEP schemes. Contrary to the BEP method with PMLs, however, our solutions are continuous everywhere; no discontinuities appear on the interfaces between slices, which is a problem for BEP especially when using a low number of modes, and the peaks near the PML boundaries that can appear in standard BEP [29] have not been observed in the current results.

As an alternative to the numerical solution presented here, a semianalytic solution method for structures with piecewise constant rectangular refractive index distributions could be established, that might be more efficient than the present numerical implementation of the algorithm for those specific cases.

The present numerical variant, however, also serves to obtain experiences for an extension of the approach towards 3D. After a dimensionality reduction using vectorial slab modes, systems of partial differential equations emerge in two spatial dimensions, which require a numerical treatment anyway. More detailed discussion follows in Chapter 5. 


\section{D scattering problems}

We develop a method for 3D-to-2D dimensionality reduction of scattering problems in photonics, based on a global slab mode expansion. The technique utilizes a variational technique, based on the vectorial 3D Maxwell's equations. One may use one single mode in the expansion, where, contrary to the 'standard' Effective Index Method, the effective parameters of the reduced problem are always rigorously defined. Adding more modes to the expansion increases the accuracy and enables radiation from the structure to also be taken into account. Calculations on a straight silicon on insulator waveguide validate the method. Results for a photonic crystal slab waveguide show that this approach predicts the location of the bandgap and other spectral features much more precisely than any 'standard' EIM approximation.

\subsection{Introduction}

In the design of photonic integrated circuits, computational tools that calculate the response of a three-dimensional structure to a given influx of light are needed. However, fully vectorial 3D simulations of scattering problems in photonic components are often almost prohibitively CPU-time and memory intensive, so one would opt for reduced models that capture the essence of the full 3D structure, while being computationally much more efficient; especially while scanning the parameter space of the device, approximate results may be sufficiently good. In later stages of a design process, more rigorous calculations would be used for the verification and fine-tuning of 
the device.

A popular technique to perform approximate calculations is the so-called Effective Index Method (EIM) [21], [110], [78], which reduces simulations of $3 \mathrm{D}$ structures to two spatial dimensions by calculating effective parameters using local guided modes. However, frequently, as is the case for photonic crystal slabs (Figure 5.1, left), no guided mode exists in a cross-section and the effective parameters for the 2D simulation are only rather ambiguously defined, i.e. rely more or less on guesswork.
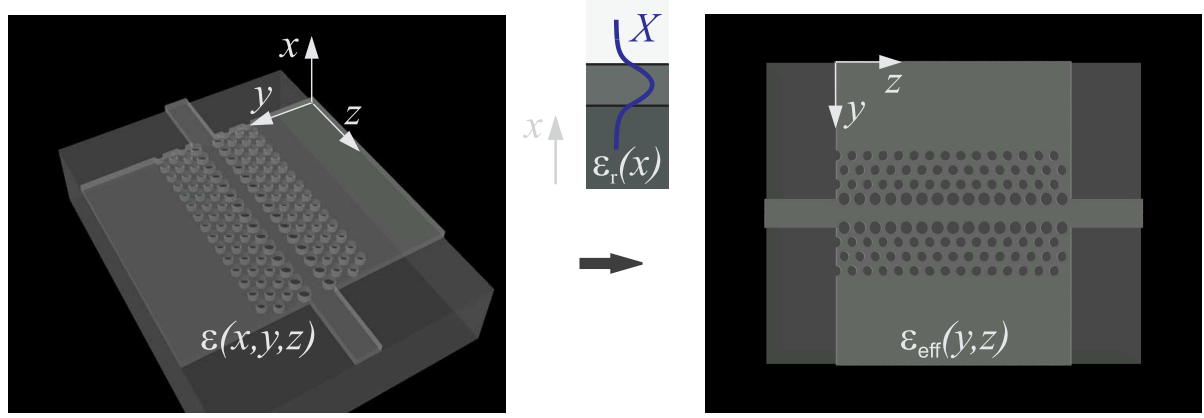

Figure 5.1: By using a TE slab mode of a reference slice with permittivity $\varepsilon_{r}(x)$ as an approximation for the $x$-dependence of the field, the simulation of wave propagation in the structure is reduced from $3 D$ to $2 D$.

More rigorous methods are mostly purely numerical (e.g. the Finite Element or Finite Difference Method), or based on expansions in the modes of 2D cross-sections (Eigenmode Expansion Method [26]). A commonly used technique is Finite Difference Time Domain (FDTD) [97], which directly solves the time evolution of Maxwell's equations.

This chapter proposes a simulation method for vectorial 3D frequency domain optical scattering problems. It reduces the spatial dimensionality of the problem by expanding the vectorial field along one spatial dimension into the modes of one or more slab waveguides (1D refractive index profiles), typically vertical cross-sections through the 3D structure at locations where one expects most of the optical field to be concentrated. The same expansion is utilized everywhere in the domain. Subsequently, a variational procedure leads to the equations for the amplitudes of all vector components of these slab modes in the other two spatial dimensions, which are subsequently reduced to a system involving just two components. A finite element scheme 
combined with Perfectly Matched Layers (PMLs) [3], [14] and Transparent Influx Boundary Conditions (TIBC) [68], [89], [94] is employed to solve the resulting equations.

When using only one slab mode in the expansion, the computational effort of the presented method is comparable to that of a standard EIM. However, contrary to the EIM, the coefficients in the resulting 2D equations are rigorously defined a priori, even when no guided mode exists in some cross-section. In order to also model interactions between vertical modes, and radiation leaving the structure vertically, more modes are needed in the expansion - increasing both the computational effort and the accuracy of the method.

In many ways, this chapter can be seen as a generalization of work reported in the previous chapters and published in [40], [36], [43], [44] and [48], dealing with a vectorial mode solver and a 2D scattering solver. Both methods are based on the same expansion and variational formalism as presented in this chapter, and the equations resulting from the current 3D scattering problem can be transformed into the ones relevant for the two aforementioned methods by applying the proper restrictions - in both cases requiring that the structure is uniform in the $z$-direction, with the additional restriction that for the $2 \mathrm{D}$ scattering solver the fields also do not depend on $z$ (i.e. that propagation happens purely in the $x-y$ plane). After the interior equations are established, different boundary conditions further distinguish the mode and 2D scattering solvers. Furthermore, the mode analysis procedure from Chapter 3 is needed in the current work to implement the transparent boundary conditions.

\subsection{Variational form of 3D scattering prob- lems in optics}

The time-harmonic propagation of a given optical influx with frequency $\omega$ through a linear, charge and current free medium, characterized by the permittivity distribution $\varepsilon(x, y, z)$, is governed by the Maxwell's curl equations

$$
\nabla \times \mathbf{E}=-\mathrm{i} \omega \mu_{0} \mu \mathbf{H}, \quad \nabla \times \mathbf{H}=\mathrm{i} \omega \varepsilon_{0} \varepsilon \mathbf{E},
$$

for the electric and magnetic fields $\mathbf{E}=\left(E_{x}, E_{y}, E_{z}\right)$ and $\mathbf{H}=\left(H_{x}, H_{y}, H_{z}\right)$ respectively. $\varepsilon_{0}$ and $\mu_{0}$ are the vacuum permittivity and permeability. The relative permeability $\mu$ is assumed to be one, as is appropriate for most 
materials at optical frequencies. Solutions $(\mathbf{E}, \mathbf{H})$ of equations (5.2.1) are stationary points of the functional [101]

$\mathfrak{F}(\mathbf{E}, \mathbf{H})=\int\left(\mathbf{E} \cdot(\nabla \times \mathbf{H})+\mathbf{H} \cdot(\nabla \times \mathbf{E})-\mathrm{i} \omega \varepsilon_{0} \varepsilon \mathbf{E}^{2}+\mathrm{i} \omega \mu_{0} \mu \mathbf{H}^{2}\right) d x d y d z$

In the following we will work with this variational formulation only.

\subsection{General approximation}

We expand each field component $F \in\left\{E_{x}, E_{y}, E_{z}, H_{x}, H_{y}, H_{z}\right\}$ of the vectorial field profiles $\mathbf{E}$ and $\mathbf{H}$ as a superposition of some known functions $X_{j}^{F}(x)$ of the $x$-coordinate, times some unknown functions $P_{j}^{F}(y, z)$, which basically define the amplitudes of the corresponding components $X_{j}^{F}(x)$ everywhere on the plane $(y, z)$ :

$$
F(x, y, z)=\sum_{j} X_{j}^{F}(x) P_{j}^{F}(y, z)
$$

Just as for the vectorial mode solver in Chapter 3, for the functions $X_{j}^{F}$ we choose certain components of slab modes. These modes are computed for a number (one or several) of slab waveguides that are defined by vertical permittivity distributions $\varepsilon_{\mathrm{r}}(x)$ at some locations in the $(y, z)$-plane. In the following these special waveguides we will call reference $(\mathrm{r})$ waveguides (Figure 5.1). For the moment we will assume that we know all necessary information about the functions $X_{j}^{F}$ and derive the equations for the coefficient-functions $P_{j}^{F}$ using the variational methods [101], [31].

By inserting the approximated field (5.3.3) into the functional (5.2.2) and by taking variations with respect to all unknown functions $P_{j}^{F}$, a system of first order differential equations for $\mathbf{P}^{F}$ - the vector of all unknown functions $P_{j}^{F}$ related to the component $F$ - emerges: 


$$
\begin{aligned}
\mathbf{A}_{11} \frac{\partial}{\partial y} \mathbf{P}^{H_{z}}+\mathbf{A}_{12} \frac{\partial}{\partial z} \mathbf{P}^{H_{y}}+\mathbf{A}_{13} \mathbf{P}^{E_{x}} & =\mathbf{0} \\
\mathbf{A}_{21} \mathbf{P}^{H_{z}}+\mathbf{A}_{22} \frac{\partial}{\partial z} \mathbf{P}^{H_{x}}+\mathbf{A}_{23} \mathbf{P}^{E_{y}} & =\mathbf{0} \\
\mathbf{A}_{31} \mathbf{P}^{H_{y}}+\mathbf{A}_{32} \frac{\partial}{\partial y} \mathbf{P}^{H_{x}}+\mathbf{A}_{33} \mathbf{P}^{E_{z}} & =\mathbf{0} \\
\mathbf{A}_{41} \frac{\partial}{\partial} \mathbf{P}^{E_{z}}+\mathbf{A}_{42} \frac{\partial}{\partial z} \mathbf{P}^{E_{y}}+\mathbf{A}_{43} \mathbf{P}^{H_{x}} & =\mathbf{0} \\
\mathbf{A}_{51} \mathbf{P}^{E_{z}}+\mathbf{A}_{52} \frac{\partial}{\partial z} \mathbf{P}^{E_{x}}+\mathbf{A}_{53} \mathbf{P}^{H_{y}} & =\mathbf{0} \\
\mathbf{A}_{61} \mathbf{P}^{E_{y}}+\mathbf{A}_{62} \frac{\partial}{\partial y} \mathbf{P}^{E_{x}}+\mathbf{A}_{63} \mathbf{P}^{H_{z}} & =\mathbf{0}
\end{aligned}
$$

The elements of the matrices $\mathbf{A}$ are of the following form:

$$
\begin{array}{lrr}
\mathbf{A}_{11}(k, j)=\left\langle X_{k}^{E_{x}}, X_{j}^{H_{z}}\right\rangle & \mathbf{A}_{12}(k, j)=-\left\langle X_{k}^{E_{x}}, X_{j}^{H_{y}}\right\rangle & \mathbf{A}_{13}(k, j)=-\mathrm{i} \omega\left\langle X_{k}^{E_{x}}, \varepsilon X_{j}^{E_{x}}\right\rangle \\
& & (5.3 .10) \\
\mathbf{A}_{21}(k, j)=-\left\langle X_{k}^{E_{y}},\left(X_{j}^{H_{z}}\right)^{\prime}\right\rangle & \mathbf{A}_{22}(k, j)=\left\langle X_{k}^{E_{y}}, X_{j}^{H_{x}}\right\rangle & \mathbf{A}_{23}(k, j)=-\mathrm{i} \omega\left\langle X_{k}^{E_{y}}, \varepsilon X_{j}^{E_{y}}\right\rangle \\
& & (5.3 .11) \\
\mathbf{A}_{31}(k, j)=\left\langle X_{k}^{E_{z}},\left(X_{j}^{H_{y}}\right)^{\prime}\right\rangle & \mathbf{A}_{32}(k, j)=-\left\langle X_{k}^{E_{z}}, X_{j}^{H_{x}}\right\rangle & \mathbf{A}_{33}(k, j)=-\mathrm{i} \omega\left\langle X_{k}^{E_{z}}, \varepsilon X_{j}^{E_{z}}\right\rangle \\
& & (5.3 .12) \\
\mathbf{A}_{41}(k, j)=\left\langle X_{k}^{H_{x}}, X_{j}^{E_{z}}\right\rangle & \mathbf{A}_{42}(k, j)=-\left\langle X_{k}^{H_{x}}, X_{j}^{E_{y}}\right\rangle & \mathbf{A}_{43}(k, j)=\mathrm{i} \omega \mu\left\langle X_{k}^{H_{x}}, X_{j}^{H_{x}}\right\rangle \\
(5.3 .13) & \\
\mathbf{A}_{51}(k, j)=-\left\langle X_{k}^{H_{y}},\left(X_{j}^{E_{z}}\right)^{\prime}\right\rangle & \mathbf{A}_{52}(k, j)=\left\langle X_{k}^{\left.H_{y}, X_{j}^{E_{x}}\right\rangle}\right. & \mathbf{A}_{53}(k, j)=\mathrm{i} \omega \mu\left\langle X_{k}^{H_{y}}, X_{j}^{H_{y}}\right\rangle \\
& & (5.3 .14) \\
\mathbf{A}_{61}(k, j)=\left\langle X_{k}^{H_{z}},\left(X_{j}^{E_{y}}\right)^{\prime}\right\rangle & \mathbf{A}_{62}(k, j)=-\left\langle X_{k}^{H_{z}}, X_{j}^{E_{x}}\right\rangle & \mathbf{A}_{63}(k, j)=\mathrm{i} \omega \mu\left\langle X_{k}^{H_{z}}, X_{j}^{H_{z}}\right\rangle
\end{array}
$$

All brackets $\langle$,$\rangle here mean an integration over x$ only: $\langle a, b\rangle=\int a b d x$, the dashes ' denote derivatives. Note that only matrices $\mathbf{A}_{13}, \mathbf{A}_{23}$ and $\mathbf{A}_{33}$ depend on the $y$ and $z$-coordinates, the other matrices are constant everywhere in $(y, z)$-plane.

Continuity conditions for

$$
n_{y} \mathbf{A}_{11} \mathbf{P}^{H_{z}}+n_{z} \mathbf{A}_{12} \mathbf{P}^{H_{y}}, \quad n_{z} \mathbf{A}_{22} \mathbf{P}^{H_{x}}, \quad n_{y} \mathbf{A}_{32} \mathbf{P}^{H_{x}}
$$

and

$$
n_{y} \mathbf{A}_{41} \mathbf{P}^{E_{z}}+n_{z} \mathbf{A}_{42} \mathbf{P}^{E_{y}}, \quad n_{z} \mathbf{A}_{52} \mathbf{P}^{E_{x}}, \quad n_{y} \mathbf{A}_{62} \mathbf{P}^{E_{x}}
$$


at dielectric interfaces also follow from the restriction procedure. The vector $\left(n_{y}, n_{z}\right)$ denotes a normal to the interface in the $(y, z)$-plane.

\subsection{Reduced problem}

Due to common fabrication technologies the light in integrated optics components mainly propagates along the initial substrate, which in our case is parallel to $(y, z)$-plane, but in general in that plane without any preferred direction of propagation. Therefore we choose to approximate those components of electric (and magnetic) field that lie in this plane by the same functions $X$, meaning that we choose the functions $X_{j}^{E_{y}}$ the same as $X_{j}^{E_{z}}$, and $X_{j}^{H_{y}}$ the same as $X_{j}^{H_{z}}$. Hence the following relations between the matrices $\mathbf{A}$ arise:

$$
\begin{gathered}
\mathbf{A}_{12}^{\top}=-\mathbf{A}_{52}=\mathbf{A}_{62}=-\mathbf{A}_{11}^{\top}, \\
\mathbf{A}_{31}=-\mathbf{A}_{21}, \quad \mathbf{A}_{33}=\mathbf{A}_{23} ; \\
\mathbf{A}_{42}^{\top}=-\mathbf{A}_{22}=\mathbf{A}_{32}=-\mathbf{A}_{41}^{\top}, \\
\mathbf{A}_{61}=-\mathbf{A}_{51}, \quad \mathbf{A}_{63}=\mathbf{A}_{53} .
\end{gathered}
$$

Interestingly it turns out that by algebraic manipulations the system of first order differential equations for six unknown vector functions (5.3.4)(5.3.9), can be reduced to a system of second order differential equations for only two unknown vector functions. Additionally using the matrix relations (5.4.18)-(5.4.21), the reduced system reads

$$
\frac{\partial}{\partial_{y}}\left(\mathbf{D}_{1} \frac{\partial}{\partial_{y}} \mathbf{u}-\mathbf{D}_{2} \frac{\partial}{\partial_{z}} \mathbf{u}\right)+\frac{\partial}{\partial_{z}}\left(\mathbf{D}_{1} \frac{\partial}{\partial z} \mathbf{u}+\mathbf{D}_{2} \frac{\partial}{\partial_{y}} \mathbf{u}\right)+\mathbf{D}_{3} \mathbf{u}=\mathbf{0},
$$

or, using a more compact notation,

$$
\nabla \cdot(\mathbf{D} \nabla \mathbf{u})+\mathbf{D}_{3} \mathbf{u}=\mathbf{0}
$$

where

$$
\mathbf{u}=\left(\begin{array}{c}
\mathbf{P}^{E_{x}} \\
\mathbf{P}^{H_{x}}
\end{array}\right), \quad \mathbf{D}=\left(\begin{array}{cc}
\mathbf{D}_{1} & -\mathbf{D}_{2} \\
\mathbf{D}_{2} & \mathbf{D}_{1}
\end{array}\right)
$$

and block-(anti)-diagonal matrices $\mathbf{D}_{1,2}$ and 3 have the form

$$
\mathbf{D}_{1}=\left(\begin{array}{cc}
\mathbf{B}_{1} & \mathbf{0} \\
\mathbf{0} & \mathbf{C}_{1}
\end{array}\right), \quad \mathbf{D}_{2}=\left(\begin{array}{cc}
\mathbf{0} & \mathbf{B}_{2} \\
\mathbf{C}_{2} & \mathbf{0}
\end{array}\right), \quad \mathbf{D}_{3}=\left(\begin{array}{cc}
\mathbf{B}_{3} & \mathbf{0} \\
\mathbf{0} & \mathbf{C}_{3}
\end{array}\right),
$$


where

$$
\begin{aligned}
& \mathbf{B}_{1}=\mathbf{A}_{52}^{\top}\left(\mathbf{A}_{53}+\mathbf{A}_{51} \mathbf{A}_{23}^{-1} \mathbf{A}_{21}\right)^{-1} \mathbf{A}_{52}, \\
& \mathbf{B}_{2}=\mathbf{A}_{52}^{\top} \mathbf{A}_{53}^{-1} \mathbf{A}_{51}\left(\mathbf{A}_{23}+\mathbf{A}_{21} \mathbf{A}_{53}^{-1} \mathbf{A}_{51}\right)^{-1} \mathbf{A}_{22}, \\
& \mathbf{B}_{3}=\mathbf{A}_{13},
\end{aligned}
$$

and

$$
\begin{aligned}
& \mathbf{C}_{1}=\mathbf{A}_{22}^{\top}\left(\mathbf{A}_{23}+\mathbf{A}_{21} \mathbf{A}_{53}^{-1} \mathbf{A}_{51}\right)^{-1} \mathbf{A}_{22}, \\
& \mathbf{C}_{2}=\mathbf{A}_{22}^{\top} \mathbf{A}_{23}^{-1} \mathbf{A}_{21}\left(\mathbf{A}_{53}+\mathbf{A}_{51} \mathbf{A}_{23}^{-1} \mathbf{A}_{21},\right)^{-1} \mathbf{A}_{52} \\
& \mathbf{C}_{3}=\mathbf{A}_{43} .
\end{aligned}
$$

The continuity conditions (5.3.16)-(5.3.17) also turn into continuity of

$$
\mathbf{u} \text { and } \mathbf{n D} \nabla \mathbf{u},
$$

where $\mathbf{n}$ is a $m \times 2 m$ matrix, with the left-most $m \times m$ part being just a diagonal matrix with $n_{y}$ on diagonal, and, similarly, the right-most $m \times m$ part being a diagonal matrix with entries $n_{z}$.

Once the solution of the problem (5.4.23), (5.4.32) is at hand, the other functions $\mathbf{P}$ can be found as

$$
\begin{aligned}
\mathbf{P}^{E_{y}}= & -\mathbf{A}_{23}^{-1} \mathbf{A}_{21}\left(\mathbf{A}_{53}+\mathbf{A}_{51} \mathbf{A}_{23}^{-1} \mathbf{A}_{21}\right)^{-1} \mathbf{A}_{52} \frac{\partial}{\partial y} \mathbf{P}^{E_{x}}- \\
& -\left(\mathbf{A}_{23}+\mathbf{A}_{21} \mathbf{A}_{53}^{-1} \mathbf{A}_{51}\right)^{-1} \mathbf{A}_{22} \frac{\partial}{\partial z} \mathbf{P}^{H_{x}} \\
\mathbf{P}^{H_{z}}= & \left(\mathbf{A}_{53}+\mathbf{A}_{51} \mathbf{A}_{23}^{-1} \mathbf{A}_{21}\right)^{-1} \mathbf{A}_{52} \frac{\partial}{\partial y} \mathbf{P}^{E_{x}}- \\
& -\mathbf{A}_{53}^{-1} \mathbf{A}_{51}\left(\mathbf{A}_{23}+\mathbf{A}_{21} \mathbf{A}_{53}^{-1} \mathbf{A}_{51}\right)^{-1} \mathbf{A}_{22} \frac{\partial}{\partial z} \mathbf{P}^{H_{x}} \\
\mathbf{P}^{H_{y}}= & -\left(\mathbf{A}_{53}+\mathbf{A}_{51} \mathbf{A}_{23}^{-1} \mathbf{A}_{21}\right)^{-1} \mathbf{A}_{52} \frac{\partial}{\partial z} \mathbf{P}^{E_{x}}- \\
& -\mathbf{A}_{53}^{-1} \mathbf{A}_{51}\left(\mathbf{A}_{23}+\mathbf{A}_{21} \mathbf{A}_{53}^{-1} \mathbf{A}_{51}\right)^{-1} \mathbf{A}_{22} \frac{\partial}{\partial y} \mathbf{P}^{H_{x}} \\
\mathbf{P}^{E_{z}}= & -\mathbf{A}_{23}^{-1} \mathbf{A}_{21}\left(\mathbf{A}_{53}+\mathbf{A}_{51} \mathbf{A}_{23}^{-1} \mathbf{A}_{21}\right)^{-1} \mathbf{A}_{52} \frac{\partial}{\partial z} \mathbf{P}^{E_{x}}+ \\
& +\left(\mathbf{A}_{23}+\mathbf{A}_{21} \mathbf{A}_{53}^{-1} \mathbf{A}_{51}\right)^{-1} \mathbf{A}_{22} \frac{\partial}{\partial_{y}} \mathbf{P}^{H_{x}} .
\end{aligned}
$$

and the complete field consequently can be constructed according to (5.3.3). 


\subsection{Weak formulation}

To solve the system of differential equations (5.4.23) together with interface conditions (5.4.32) we will use a Finite Element Method, since it accurately takes into account the geometry of the structure. If $\Omega$ is the computational window and $\partial \Omega$ is its boundary, we can write a weak formulation of (5.4.23) as

$$
\int_{\Omega} \mathbf{v}^{\top}\left[\nabla \cdot(\mathbf{D} \nabla \mathbf{u})+\mathbf{D}_{3} \mathbf{u}\right] d \Omega-\int_{\partial \Omega} \mathbf{v}^{\top} \mathbf{n D}\left[\nabla \mathbf{u}-\nabla \mathbf{u}_{\mathrm{ext}}\right] d \partial \Omega=0
$$

where $\mathbf{v}(y, z)$ is a continuous test vector-function defined on $\Omega$, and the matrix $\mathbf{n}$ is defined as in Eqn. (5.4.32) with an outward normal $\left(n_{y}, n_{z}\right)$. Here $\mathbf{u}(y, z)$ and $\mathbf{u}_{\text {ext }}(y, z)$ are the solution of (5.4.23) in the interior and the exterior of $\Omega$ correspondingly. In the following we will choose the computational window such, that in some neighbourhood of the boundary the structure is uniform in the direction perpendicular to the boundary, meaning that the matrix $\mathbf{D}(y, z)$ does not have any jumps across $\partial \Omega$.

By requiring that the equation (5.5.37) is satisfied for an arbitrary function $\mathbf{v}(y, z)$, one recovers the system (5.4.23) together with continuity conditions (5.4.32). Integrating by parts the first term of (5.5.37) turns it into the standard weak form:

$$
\int_{\Omega}\left[-\nabla \mathbf{v}^{\top} \mathbf{D} \nabla \mathbf{u}+\mathbf{D}_{3} \mathbf{u}\right] d \Omega+\int_{\partial \Omega} \mathbf{v}^{\top} \mathbf{n} \mathbf{D} \nabla \mathbf{u}_{\mathrm{ext}} d \partial \Omega=0 .
$$

\subsection{Transparent Influx Boundary Conditions}

In the following we are going to derive transparent influx boundary conditions that allow light to leave the computational window boundaries undisturbed, with the possibility to prescribe an influx through the boundary. Since all boundaries can be treated in the same way, we will only discuss the left-hand boundary of the domain $z=l$ (Figure 5.2). We assume that any waveguides in the exterior run perpendicular to the computational window boundary. It means that the permittivity $\varepsilon(y, z)$ in the exterior cross-section is invariant in the $z$-direction and is identical to the one on the western boundary. Furthermore, in the exterior at $y=b$ and $y=t$, the bottom and top of the calculation window, $\mathbf{P}^{E_{x}}$ and $\mathbf{P}^{H_{x}}$ will be required to 
vanish. Then a particular solution of (5.4.23) in the left exterior $\mathbf{u}_{\text {ext }}(y, z)$ can be represented as

$$
\mathbf{u}_{\text {ext }}(y, z)=f \mathrm{e}^{\mathrm{i} \beta z} \mathbf{y}(y)
$$

with some coefficient $f$, and the pair $\beta$, y satisfying the following quadratic eigenvalue problem:

$$
\left(\mathbf{D}_{1} \mathbf{y}^{\prime}+\mathrm{i} \beta \mathbf{D}_{2} \mathbf{y}\right)^{\prime}+\mathbf{D}_{3} \mathbf{y}=\beta^{2} \mathbf{D}_{1} \mathbf{y}+\mathrm{i} \beta \mathbf{D}_{2} \mathbf{y}^{\prime} .
$$

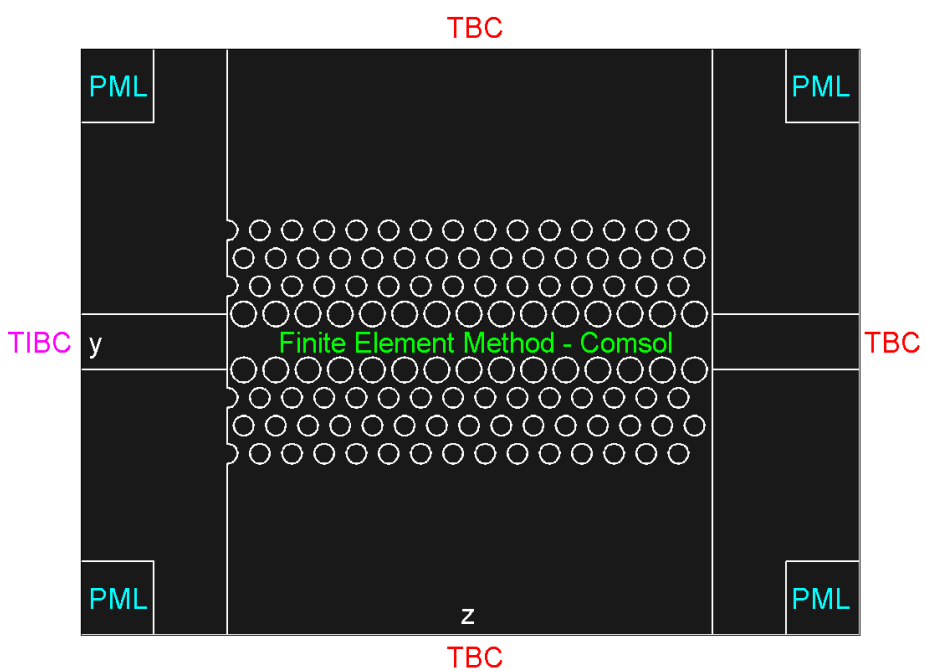

Figure 5.2: The calculation window. On the boundaries of the domain Transparent Influx Boundary Conditions are used (or Transparent Boundary Conditions if there is no influx). In the corners of the window, and extending into the exterior, we can place PML sections to absorb light hitting the corner points of the domain.

Note that the coupling between the $\mathbf{P}^{E x}$ and $\mathbf{P}^{H x}$ terms only occurs in the term with $\beta$. This eigenvalue problem is absolutely the same as in equation (20) in the vectorial mode solver paper [43] (eqn. (3.5.20) in this thesis). This is natural since basically in the exterior we are looking for vectorial modes propagating perpendicular to the computational window boundary. Solutions of this eigenvalue equation correspond to modes running in the 
positive or negative $z$-direction; we will denote those as $j_{\text {in }}$ and $j_{\text {out }}$ correspondingly. Hence the field in the left exterior can be represented as

$$
\mathbf{u}_{\text {ext }}(y, z)=\sum_{j_{\text {out }}} f_{j_{\text {out }}} \mathrm{e}^{\mathrm{i} \beta_{j_{\text {out }}} z} \mathbf{y}_{j_{\text {out }}}(y)+\sum_{j_{\text {in }}} f_{j_{\text {in }}} \mathrm{e}^{\mathrm{i} \beta_{j_{\text {in }}} z_{y_{j_{\text {in }}}}(y)}
$$

with prescribed amplitudes $f_{j_{\text {in }}}$ of the incoming field, as the influx. Or in matrix notation

$$
\mathbf{u}_{\text {ext }}(y, z)=\mathbf{Y}_{\text {out }}(y) \mathbf{E}_{\text {out }}(z) \mathbf{f}_{\text {out }}+\mathbf{Y}_{\text {in }}(y) \mathbf{E}_{\text {in }}(z) \mathbf{f}_{\text {in }},
$$

where $\mathbf{Y}_{\text {out }}(y)$ - a matrix, composed of vectors $\mathbf{y}_{j_{\text {out }}}(y)$ as columns, $\mathbf{E}_{\text {out }}(z)$ - a diagonal matrix with entries $\mathrm{e}^{\mathrm{i} \beta_{j_{\text {out }}} z}$, and $\mathbf{f}_{\text {out }}-$ a column-vector of all coefficients $f_{j_{\text {out }}}$; the same is valid for the terms with $j_{\text {in }}$.

Since the solution of (5.4.23) should be continuous, at the boundary $z=l$ the solution in the exterior $\mathbf{u}_{\mathrm{ext}}(y, z)$ should match that of the interior $\mathbf{u}(y, z)$ :

$$
\mathbf{u}(y, l)=\mathbf{Y}_{\text {out }}(y) \mathbf{E}_{\text {out }}(l) \mathbf{f}_{\text {out }}+\mathbf{Y}_{\text {in }}(y) \mathbf{E}_{\text {in }}(l) \mathbf{f}_{\text {in }} .
$$

Hence the unknown coefficients $\mathbf{f}_{\text {out }}$ could be found as a function of the solution of the interior problem at the boundary. By multiplying both sides of (5.6.43) by $\mathbf{Y}_{\text {out }}^{\top}(y)$ and integrating over $y$, one obtains

$$
\begin{aligned}
\left\{\mathbf{Y}_{\text {out }}^{\top}(y), \mathbf{u}_{\text {ext }}(y, l)\right\} & =\left\{\mathbf{Y}_{\text {out }}^{\top}(y), \mathbf{Y}_{\text {out }}(y)\right\} \mathbf{E}_{\text {out }}(l) \mathbf{f}_{\text {out }}+ \\
& +\left\{\mathbf{Y}_{\text {out }}^{\top}(y), \mathbf{Y}_{\text {in }}(y)\right\} \mathbf{E}_{\text {in }}(l) \mathbf{f}_{\text {in }},
\end{aligned}
$$

where the symbol $\{\cdot, \cdot\}$ means integration over $y$. Now, the matrix $\left\{\mathbf{Y}_{\text {out }}^{\top}(y), \mathbf{Y}_{\text {out }}(y)\right\}$ is invertible, since it is positive semi-definite, and we assume that the vectors $\mathbf{y}_{j_{\text {out }}}(y)$ are not linearly dependent. Hence multiplying both sides of (5.6.44) by the inverse of this matrix, we get the unknown amplitudes of the outgoing modal fields

$$
\begin{aligned}
\mathbf{f}_{\text {out }}= & \mathbf{E}_{\text {out }}^{-1}(l)\left\{\mathbf{Y}_{\text {out }}^{\top}(y), \mathbf{Y}_{\text {out }}(y)\right\}^{-1} . \\
& \cdot\left[\left\{\mathbf{Y}_{\text {out }}^{\top}(y), \mathbf{u}(y, l)\right\}-\left\{\mathbf{Y}_{\text {out }}^{\top}(y), \mathbf{Y}_{\text {in }}(y)\right\} \mathbf{E}_{\text {in }}(l) \mathbf{f}_{\text {in }}\right] .
\end{aligned}
$$

Now we can express the function $\nabla \mathbf{u}_{\text {ext }}(y, z)$ at the boundary $z=l$ only in terms of the interior solution $\mathbf{u}(y, z)$ also at the boundary. After differentiating (5.6.42) and using the relation (5.6.45) we can write:

$$
\nabla \mathbf{u}_{\text {ext }}(y, l)=\left(\begin{array}{c}
\mathbf{Y}_{\text {out }}(y) \boldsymbol{\Lambda}_{\text {out }} \mathbf{E}_{\text {out }}(l) \mathbf{f}_{\text {out }}+\mathbf{Y}_{\text {in }}(y) \boldsymbol{\Lambda}_{\text {in }} \mathbf{E}_{\text {in }}(l) \mathbf{f}_{\text {in }} \\
\mathbf{Y}_{\text {out }}^{\prime}(y) \mathbf{E}_{\text {out }}(l) \mathbf{f}_{\text {out }}+\mathbf{Y}_{\text {in }}^{\prime}(y) \mathbf{E}_{\text {in }}(l) \mathbf{f}_{\text {in }}
\end{array}\right)=
$$




$$
=\left(\begin{array}{l}
\mathbf{Y}_{\text {out }}(y) \boldsymbol{\Lambda}_{\text {out }}\left\{\mathbf{Y}_{\text {out }}^{\top}(y), \mathbf{Y}_{\text {out }}(y)\right\}^{-1}\left\{\mathbf{Y}_{\text {out }}^{\top}(y), \mathbf{u}(y, l)\right\}- \\
-\left[\mathbf{Y}_{\text {out }}(y) \boldsymbol{\Lambda}_{\text {out }}\left\{\mathbf{Y}_{\text {out }}^{\top}(y), \mathbf{Y}_{\text {out }}(y)\right\}^{-1} \cdot\right. \\
\left.\cdot\left\{\mathbf{Y}_{\text {out }}^{\top}(y), \mathbf{Y}_{\text {in }}(y)\right\}-\mathbf{Y}_{\text {in }}(y) \boldsymbol{\Lambda}_{\text {in }}\right] \mathbf{E}_{\text {in }}(l) \mathbf{f}_{\text {in }} \\
\mathbf{Y}_{\text {out }}^{\prime}(y)\left\{\mathbf{Y}_{\text {out }}^{\top}(y), \mathbf{Y}_{\text {out }}(y)\right\}^{-1}\left\{\mathbf{Y}_{\text {out }}^{\top}(y), \mathbf{u}(y, l)\right\}- \\
-\left[\mathbf{Y}_{\text {out }}^{\prime}(y)\left\{\mathbf{Y}_{\text {out }}^{\top}(y), \mathbf{Y}_{\text {out }}(y)\right\}^{-1} \cdot\right. \\
\left.\cdot\left\{\mathbf{Y}_{\text {out }}^{\top}(y), \mathbf{Y}_{\text {in }}(y)\right\}-\mathbf{Y}_{\text {in }}^{\prime}(y)\right] \mathbf{E}_{\text {in }}(l) \mathbf{f}_{\text {in }}
\end{array}\right)
$$

where $\boldsymbol{\Lambda}_{\text {out (in) }}$ is a diagonal matrix with entries i $\beta_{j_{\text {out (in) }}}$ and the symbol ' here means the derivative with respect to $y$.

Substitution of the expression for the $\nabla \mathbf{u}_{\text {ext }}(y, l)$ (5.6.46) into the weak formulation (5.5.38) restricts the weak formulation to the computational domain $\Omega$ only.

\subsubsection{Remarks}

The mode with propagation constant $\beta_{j}$ is an approximate mode of the outgoing waveguide and depends on the particular expansion (5.3.3). Chapter 3 contains several examples on the convergence of the approximate mode profile together with the propagation constant. Nevertheless in this chapter we will assume that the modal solution of (5.6.40) represents the true mode profile well enough, and therefore for the influx we will simply consider this modal solution.

Furthermore, it can readily be seen that inverting the sign of $\beta$ in (5.6.40) (i.e. a reversal of the propagation direction) also gives a solution - in which all the eigenfunction's $\mathbf{P}^{E x}$ also get a minus sign. Thus, contrary to the systems described in [68], [89], [31], [93], in this case the left-travelling eigenfunctions are not equal to the right-travelling ones, even though their eigenvalues are just each others negative. From a physical point of view, we can understand the fact that the eigenfunctions change - the $z$-component of the Poynting vector should, after all, change sign - which is accomplished exactly by the transformation of the eigenfunction that we observe here. 


\subsection{Choice of the expansion basis}

Similarly to the vectorial mode problem of Chapter 3, here a five component approximation (called VEIM5 in that chapter) will be used. The TE basis mode number $j$ with mode profile components $\chi_{j}^{E_{y}}, \chi_{j}^{H_{x}}, \chi_{j}^{H_{z}}$ contributes to the expansion of components $E_{y}, E_{z}, H_{x}, H_{y}$ and $H_{z}$. Likewise, the TM basis mode number $l$ with mode profile components $\chi_{l}^{E_{y}}, \chi_{l}^{H_{x}}, \chi_{l}^{H_{z}}$ contributes to the expansion of components $E_{x}, E_{y}, E_{z}, H_{y}$ and $H_{z}$, such that the complete expansion reads

$$
\begin{aligned}
& \left(\begin{array}{c}
E_{x}, E_{y}, E_{z} \\
H_{x}, H_{y}, H_{z}
\end{array}\right)(x, y, z)= \\
= & \sum_{j \in \mathrm{TE}}\left(\begin{array}{ccc}
0, & \chi_{j}^{E_{y}}(x) P_{j}^{E_{y}}(y, z), & \chi_{j}^{E_{y}}(x) P_{j}^{E_{z}}(y, z) \\
\chi_{j}^{H_{x}}(x) P_{j}^{H_{x}}(y, z), & \chi_{j}^{H_{z}}(x) P_{j}^{H_{y}}(y, z), & \chi_{j}^{H_{z}}(x) P_{j}^{H_{z}}(y, z)
\end{array}\right)+ \\
+ & \sum_{l \in \mathrm{TM}}\left(\begin{array}{ccc}
\chi_{l}^{E_{x}}(x) P_{l}^{E_{x}}(y, z), & \chi_{l}^{E_{z}}(x) P_{l}^{E_{y}}(y, z), & \chi_{l}^{E_{z}}(x) P_{l}^{E_{z}}(y, z) \\
0, & \chi_{l}^{H_{y}}(x) P_{l}^{H_{y}}(y, z), & \chi_{l}^{H_{y}}(x) P_{l}^{H_{z}}(y, z)
\end{array}\right) .
\end{aligned}
$$

Note that the electric and magnetic $y$ - and $z$-components are approximated by the same vertical functions as assumed in 5.4 .

While here we use only this type of the expansion basis, we do not claim that it represents the true solution the best. It is in principle possible to try other functions $X$ in the expansion. As an example, one might consider to use Fourier basis functions. Then the present approach would become some sort of 3D generalization of the 2D method presented in Ref. [105]. Yet another possibility is to use the three component approximation (VEIM3) outlined in Chapter 3 .

\subsection{Relation with the Effective Index Method}

In the following section we are going to show what happens if only a single, TE or TM, slab mode is taken into account. Using the variational reasoning developed above we will rigorously derive an analog to the Effective Index Method. 


\subsubsection{TE polarization}

Let us consider only one TE slab mode propagating in the direction $z$ with propagation constant $\beta_{\mathrm{r}}$ from a reference slice with permittivity distribution $\varepsilon_{\mathrm{r}}(x)$ :

$$
\left(\begin{array}{c}
E_{x}, E_{y}, E_{z} \\
H_{x}, H_{y}, H_{z}
\end{array}\right)_{\text {slab }}(x, z)=\left(\begin{array}{ccc}
0, & \chi^{E_{y}}(x), & 0 \\
\chi^{H_{x}}(x), & 0, & \chi^{H_{z}}(x)
\end{array}\right) \cdot \mathrm{e}^{-\mathrm{i} \beta_{\mathrm{r}} z},
$$

The principal electric component $\chi^{E_{y}}$ satisfies the equation

$$
\left(\chi^{E_{y}}(x)\right)^{\prime \prime}+k^{2} \varepsilon_{\mathrm{r}}(x) \chi^{E_{y}}(x)=\beta_{\mathrm{r}}^{2} \chi^{E_{y}}(x)
$$

with vacuum wavenumber $k=2 \pi / \lambda$. The remaining two nonzero components of the mode profile can be derived directly from $\chi^{E_{y}}$.

We assume that this vertical shape constitutes an adequate approximation for the (polarized) optical field in the 3D structure

$$
\begin{aligned}
& \left(\begin{array}{c}
E_{x}, E_{y}, E_{z} \\
H_{x}, H_{y}, H_{z}
\end{array}\right)_{\text {complete }}(x, y, z)= \\
& \quad=\left(\begin{array}{ccc}
0, & \chi^{E_{y}}(x) P^{E_{y}}(y, z), & \chi^{E_{y}}(x) P^{E_{z}}(y, z) \\
\chi^{H_{x}}(x) P^{H_{x}}(y, z), & \chi^{H_{z}}(x) P^{H_{y}}(y, z), & \chi^{H_{z}}(x) P^{H_{z}}(y, z)
\end{array}\right),
\end{aligned}
$$

with some unknown functions $P$. Note that the $y$ - and $z$-components of the electromagnetic field are approximated by the same functions $\chi$.

Using the relations between the slab mode components, from (5.4.23) it follows that $P^{H_{x}}$ satisfies the following second order differential equation

$$
\left(\partial_{y} \frac{1}{\varepsilon_{\mathrm{eff}}(y, z)} \partial_{y}+\partial_{z} \frac{1}{\varepsilon_{\mathrm{eff}}(y, z)} \partial_{z}+k^{2}\right) P^{H_{x}}(y, z)=0
$$

with

$$
\varepsilon_{\mathrm{eff}}(y, z)=\frac{\beta_{\mathrm{r}}^{2}}{k^{2}}+\frac{\int\left(\varepsilon(x, y, z)-\varepsilon_{\mathrm{r}}(x)\right)\left(\chi^{E_{y}}(x)\right)^{2} d x}{\int\left(\chi^{E_{y}}(x)\right)^{2} d x} .
$$

This looks exactly like a 2D TM Helmholtz equation with (effective) permittivity $\varepsilon_{\text {eff }}$, and similar to what is used in the standard Effective Index Method. In the reference slice, where $\varepsilon(x, y, z)=\varepsilon_{\mathrm{r}}(x)$, the effective 
permittivity is equal to the squared effective mode index $\beta_{\mathrm{r}}^{2} / k^{2}$. Elsewhere, however, this squared effective index is modified by the difference between the local permittivity and that of the reference slice, weighted by the local intensity of the major component of the reference mode profile. Hence, contrary to the EIM, even in slices where no guided mode exists the effective indices are still rigorously defined.

Note that the original problem (5.2.1) deals with six unknown field components, each depending on three spatial coordinates. The present approximation reduced it to a single function of two spatial coordinates only.

All other field components are related to $P^{H_{x}}$ in the following manner (5.4.33) - (5.4.36):

$$
\left(\begin{array}{c}
P^{E_{x}}, P^{E_{y}}, P^{E_{z}} \\
P^{H_{x}}, P^{H_{y}}, P^{H_{z}}
\end{array}\right)(y, z)=\frac{\mathrm{i} \beta_{\mathrm{r}}}{k^{2} \varepsilon_{\mathrm{eff}}}\left(\begin{array}{ccc}
0, & \partial_{z} P^{H_{x}}, & -\partial_{y} P^{H_{x}} \\
\left(-\mathrm{i} k^{2} \varepsilon_{\mathrm{eff}} / \beta_{\mathrm{r}}\right) P^{H_{x}}, & \partial_{y} P^{H_{x}}, & \partial_{z} P^{H_{x}}
\end{array}\right) .
$$

Equation (5.8.53) permits a quite intuitive interpretation. Inside each homogeneous region a partial solution of (5.8.51) is

$$
P^{H_{x}}(y, z)=c \mathrm{e}^{-\mathrm{i}\left(k_{y} y+k_{z} z\right)}, \quad \text { with } \quad k_{y}^{2}+k_{z}^{2}=k^{2} \varepsilon_{\text {eff }} .
$$

If we define $\rho^{2}=k^{2} \varepsilon_{\text {eff }}$, and an angle $\theta$ such that $\cos \theta=k_{z} / \rho$ and substitute it in (5.8.53), we obtain

$$
\begin{aligned}
& \left(\begin{array}{c}
P^{E_{x}}, P^{E_{y}}, P^{E_{z}} \\
P^{H_{x}}, P^{H_{y}}, P^{H_{z}}
\end{array}\right)(y, z)= \\
& \quad=c \frac{\beta_{\mathrm{r}}}{\rho} \mathrm{e}^{-\mathrm{i} \rho(-\sin \theta y+\cos \theta z)}\left(\begin{array}{ccc}
0, & \cos \theta, & \sin \theta \\
\rho / \beta_{\mathrm{r}}, & -\sin \theta, & \cos \theta
\end{array}\right) .
\end{aligned}
$$

In the reference slice we have $\rho=\beta_{\mathrm{r}}$, and hence the functions $P$ act as a rotation of the slab mode around the $x$-axis. In all other regions, in addition to the rotation of the $y$ - and $z$-components, the $x$-component is scaled by $\rho / \beta_{\mathrm{r}}$.

Moreover, as (5.8.55) is only a partial solution and the fundamental solution of (5.8.51) is a superposition of partial solutions, the functions $P$ (5.8.53) act as a superposition of the slab mode $\chi$ rotated around the $x$-axis by different angles $\theta$.

\subsubsection{TM polarization}

Now we will take only one TM slab mode propagating in the direction $z$ with propagation constant $\beta_{\mathrm{r}}$ from the reference slice $\mathrm{r}$, which has permittivity 
distribution $\varepsilon_{\mathrm{r}}(x)$ :

$$
\left(\begin{array}{c}
E_{x}, E_{y}, E_{z} \\
H_{x}, H_{y}, H_{z}
\end{array}\right)_{\text {slab }}(x, z)=\left(\begin{array}{ccc}
\chi^{E_{x}}(x), & 0, & \chi^{E_{z}}(x) \\
0, & \chi^{H_{y}}(x), & 0
\end{array}\right) \cdot \mathrm{e}^{-\mathrm{i} \beta_{\mathrm{r}} z},
$$

with function $\chi^{H_{y}}$ satisfying the TM slab mode equation

$$
\left(\frac{1}{\varepsilon_{\mathrm{r}}(x)}\left(\chi^{H_{y}}(x)\right)^{\prime}\right)^{\prime}+k^{2} \chi^{H_{y}}(x)=\beta_{\mathrm{r}} \frac{1}{\varepsilon_{\mathrm{r}}(x)} \chi^{H_{y}}(x) .
$$

The vectorial field profile everywhere is written as

$$
\begin{aligned}
& \left(\begin{array}{c}
E_{x}, E_{y}, E_{z} \\
H_{x}, H_{y}, H_{z}
\end{array}\right) \quad(x, y, z)= \\
& \quad=\left(\begin{array}{ccc}
\chi^{E_{x}}(x) P^{E_{x}}(y, z), & \chi^{E_{z}}(x) P^{E_{y}}(y, z), & \chi^{E_{z}}(x) P^{E_{z}}(y, z) \\
0, & \chi^{H_{y}}(x) P^{H_{y}}(y, z), & \chi^{H_{y}}(x) P^{H_{z}}(y, z)
\end{array}\right) .
\end{aligned}
$$

with some unknown functions $P$. Again the $y$ and $z$ components of the electromagnetic field are approximated by the same functions $\chi$.

Using the relations between the components of the slab mode $\chi$ one can find from (5.4.23) that the function $P^{E_{x}}$ satisfies the following second order differential equation

$$
\left(\partial_{y} \frac{1}{\varepsilon_{1 \mathrm{eff}}(y, z)} \partial_{y}+\partial_{z} \frac{1}{\varepsilon_{1 \mathrm{eff}}(y, z)} \partial_{z}+k^{2} \varepsilon_{2}(y, z)\right) P^{E_{x}}(y, z)=0
$$

with

$$
\begin{aligned}
\varepsilon_{1 \mathrm{eff}}(y, z) & =\frac{\beta_{\mathrm{r}}^{2}}{k^{2}} \frac{\int \varepsilon_{\mathrm{r}}(x)\left(\chi^{E_{z}}(x)\right)^{2} d x}{\int \varepsilon(x, y, z)\left(\chi^{E_{z}}(x)\right)^{2} d x}+ \\
& +\frac{\int\left(\chi^{H_{y}}(x)\right)^{2} d x}{\int \frac{1}{\varepsilon_{\mathrm{r}}(x)}\left(\chi^{H_{y}}(x)\right)^{2} d x} \frac{\int\left(\varepsilon(x, y, z)-\varepsilon_{\mathrm{r}}(x)\right)\left(\chi^{E_{z}}(x)\right)^{2} d x}{\int \varepsilon(x, y, z)\left(\chi^{E_{z}}(x)\right)^{2} d x},
\end{aligned}
$$

$$
\varepsilon_{2}(y, z)=\frac{\int \varepsilon(x, y, z)\left(\chi^{E_{x}}(x)\right)^{2} d x}{\int \varepsilon_{\mathrm{r}}(x)\left(\chi^{E_{x}}(x)\right)^{2} d x}
$$


This does not look like a TE or TM Helmholtz equation as the permittivity distribution appears both under the derivative sign and in front of the term without any derivative. So here is one of the major differences with the EIM. In the reference slice the relative permittivity is equal to that of the reference slice $\left(\varepsilon(x, y, z)=\varepsilon_{\mathrm{r}}(x)\right)$ and hence the effective relative permittivity $\varepsilon_{\text {leff }}(y, z)=\beta_{\mathrm{r}}^{2} / k^{2}$ is equal to the effective index squared of the mode of the reference slice, and $\varepsilon_{2}(y, z)=1$. Again, contrary to the EIM, even in slices where no guided mode exists, the effective indices are still rigorously defined.

Now all the other field components can be related to the $P^{E_{x}}$ in the following manner

$$
\begin{aligned}
& \left(\begin{array}{c}
P^{E_{x}}, P^{E_{y}}, P^{E_{z}} \\
P^{H_{x}}, P^{H_{y}}, P^{H_{z}}
\end{array}\right)(y, z)= \\
& \quad=\frac{\mathrm{i} \beta_{\mathrm{r}}}{k^{2} \varepsilon_{1 \mathrm{eff}}}\left(\begin{array}{ccc}
\left(-\mathrm{i} k^{2} \varepsilon_{1 \mathrm{eff}} / \beta_{\mathrm{r}}\right) P^{E_{x}}, & \varepsilon_{3}^{-1} \partial_{y} P^{E_{x}}, & \varepsilon_{3}^{-1} \partial_{z} P^{E_{x}} \\
0, & \partial_{z} P^{E_{x}}, & -\partial_{y} P^{E_{x}}
\end{array}\right),
\end{aligned}
$$

where $\varepsilon_{3}(y)=\frac{\int \varepsilon(x, y, z)\left(\chi^{E_{z}}(x)\right)^{2} d x}{\int \varepsilon_{\mathrm{r}}(x)\left(\chi^{E_{z}}(x)\right)^{2} d x}$.

Next we will show what (5.8.62) means and how it acts on the slab mode $\chi$. One can show that inside each homogeneous region a partial solution of (5.8.59) will be

$$
P^{H_{x}}=c \mathrm{e}^{-\mathrm{i}\left(k_{y} y+k_{z} z\right)}, \quad \text { with } \quad k_{y}^{2}+k_{z}^{2}=k^{2} \varepsilon_{1 \mathrm{eff}} \varepsilon_{2} .
$$

If we define $\rho^{2}=k^{2} \varepsilon_{1 \text { eff }} \varepsilon_{2}$ and angle $\theta$ such that $\cos \theta=k_{z} / \rho$ and substitute it in (5.8.62) we get

$$
\begin{aligned}
& \left(\begin{array}{c}
P^{E_{x}}, P^{E_{y}}, P^{E_{z}} \\
P^{H_{x}}, P^{H_{y}}, P^{H_{z}}
\end{array}\right)(y, z)= \\
& =c \frac{\beta_{\mathrm{r}} \varepsilon_{2}}{\rho} \mathrm{e}^{-\mathrm{i} \rho(-\sin \theta y+\cos \theta z)}\left(\begin{array}{ccc}
\rho / \beta_{\mathrm{r}} \varepsilon_{2}, & -\varepsilon_{3}^{-1} \sin \theta, & \varepsilon_{3}^{-1} \cos \theta \\
0, & \cos \theta, & \sin \theta
\end{array}\right) .
\end{aligned}
$$

It appears that in the reference slice, $\rho=\beta_{\mathrm{r}}$ and $\varepsilon_{3}=1$, and hence functions $P$ act as a rotation of a slab mode. In all the other slices in addition to the rotation of all the $y$ and $z$ components of the slab mode, the $x$ component gets scaled by $\rho / \beta_{\mathrm{r}} \varepsilon_{2}$ and $E_{y}$ and $E_{z}$ by $\varepsilon_{3}^{-1}$.

Moreover, as (5.8.64) is only a partial solution of (5.8.59) and the fundamental solution of (5.8.59) is a superposition of partial solutions, the func- 
tions $P$ (5.8.62) act as a superposition of the slab mode $\chi$ rotated around the $x$-axis.

\section{$5.9 \quad$ Numerical results}

In this section we will validate the proposed method by means of simulations on two examples. First, a simple straight silicon on insulator waveguide; second, a photonic crystal slab waveguide.

To solve the system (5.4.23), (5.4.32) in the interior, as well as to calculate the modes of the exterior, we use the commercial finite element package COMSOL. The calculation window looks as shown in Figure 5.2. The procedure is as follows: First, the slab modes are calculated, either semianalytically or by means of finite elements, where at the boundaries in $x$ Perfectly Matched Layers are employed like in Chapter 4. Then, the finite element equations are set up for the interior, and the resulting matrix equations (still assuming Neumann boundary conditions) are generated. Next, the modes of the exterior are calculated, and the modifications to the finite element matrices are applied to implement the transparent influx boundary conditions. Finally, the matrix equation is solved and the resulting fields are extracted and analyzed.

\subsubsection{Silicon on insulator waveguide}

The first example that we will consider is a straight silicon-on-insulator waveguide; this simple structure is chosen to validate the method and to show its consistency with respect to the mode solver procedures of Chapter 3. Its geometry is shown in Figure 5.3. We will only consider input into the fundamental TE mode, and will only use TE slab modes in the expansion. We will examine the effects of the finite element grid size for the situation with one slab mode in the expansion, and also observe the fields with three modes in the expansion. All slab modes will be taken from the waveguide core region. 


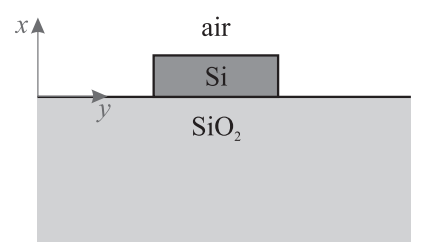

Figure 5.3: The silicon on insulator waveguide considered in this section. Its width is $0.44 \sqrt{3} \mu \mathrm{m}$ and its thickness is $220 \mathrm{~nm}$. The wavelength considered is $1.55 \mu \mathrm{m}$. The refractive index of the silicon is taken to be $\sqrt{12.1}$, of the silicon dioxide it is 1.445, and air has 1.0. Slab modes are calculated on a window $x \in[-1.3,1.5] \mu m$ using 480 linear elements; the interior equations are solved on a calculation window $y \in[0,8] \mu m, z \in[0,8] \mu m$ (cf. Figs. 5.5 $-5.7)$.

When the finite element grid is refined, both the internal grid and the grid on the boundary (and thus the exterior) change. Since the grid on the exterior changes, the solutions and propagation constants of the modes of the exterior change. Furthermore, inside the domain, the numerical dispersion of the finite element method also modifies the simulated propagation constant. We measure the propagation constant in the interior by analyzing the phase $\phi$ of $P^{H x}$ along the center of the waveguide; after observing that along a section from $y=a$ to $y=b$ this phase decreases linearly with $y$, the effective index can be calculated as

$$
n_{\mathrm{eff}}=-\frac{1}{k} \frac{\phi(b)-\phi(a)}{b-a} .
$$

Figure 5.4 shows the effective index of the exterior mode, as well as the one calculated as in Equation (5.9.65), and a reference value obtained with a commercial vectorial mode solver [10].

As the finite element mesh is refined, the numerical dispersion obviously decreases and the interior propagation constant gets closer to the one in the exterior. Naturally, they do not converge exactly to the commercial results - as shown in Chapter 4, more modes are needed to reach that value. 


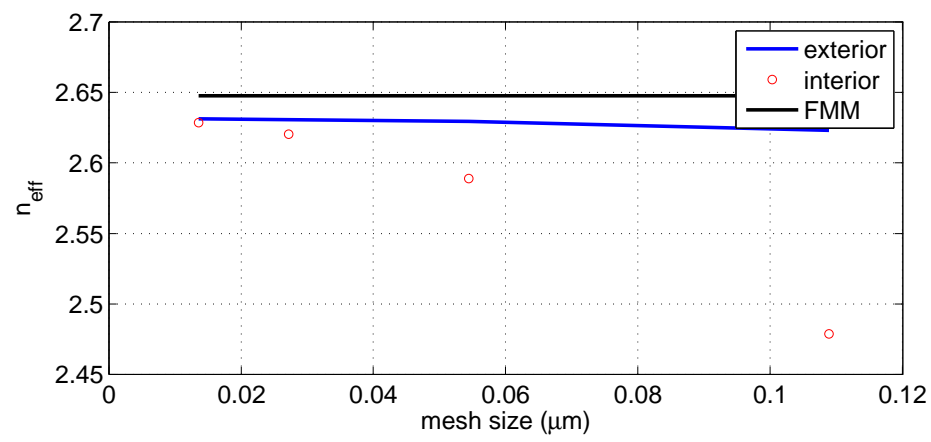

Figure 5.4: Effective index of the mode of the waveguide of Figure 5.3, calculated by solving the finite element mode equation in the exterior (denoted 'exterior'), by measuring the calculated phase evolution in the waveguide ('interior'), and using a commercial Film Mode Matching algorithm ('FMM'), versus the mesh size inside the waveguide on the boundary.
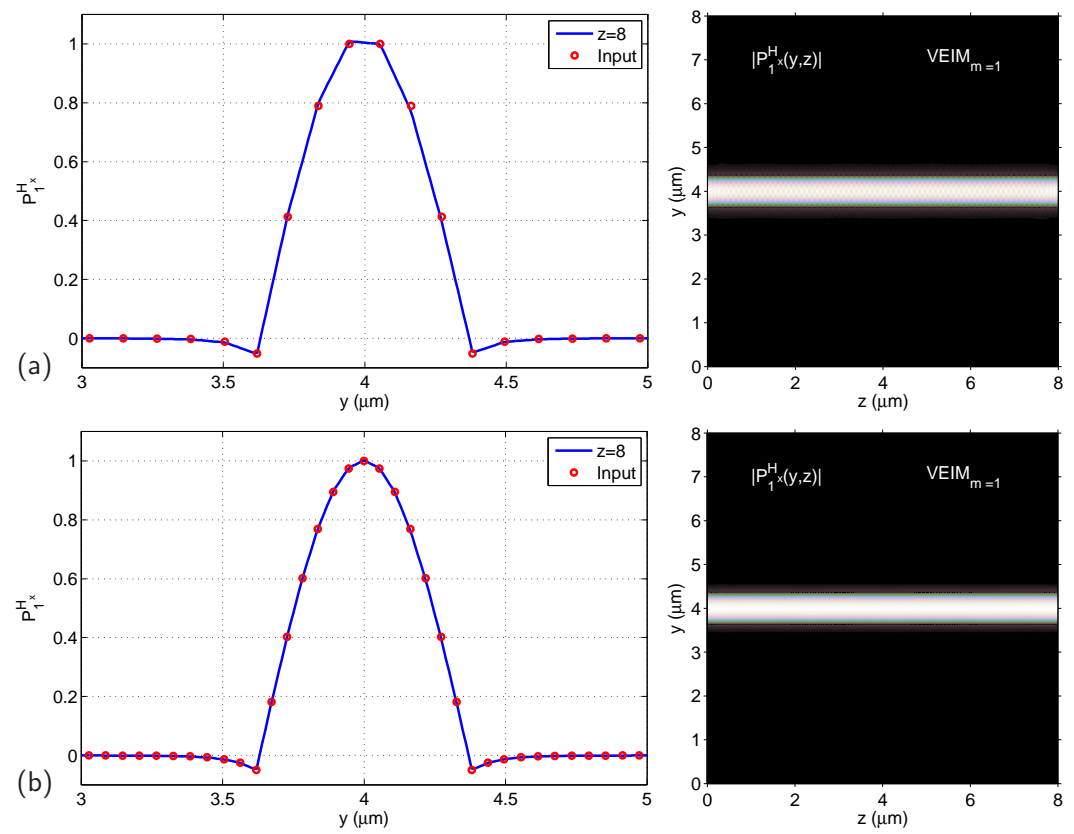

Figure 5.5: Input mode profile and field at the right-hand side of the window $(z=8 \mu \mathrm{m})$ (left column), and a top view of $\left|P_{1}^{H x}\right|$ (right column), for two mesh sizes: in (a) the mesh size in the waveguide is about $109 \mathrm{~nm}$, while in (b) it is about $54 \mathrm{~nm}$. 

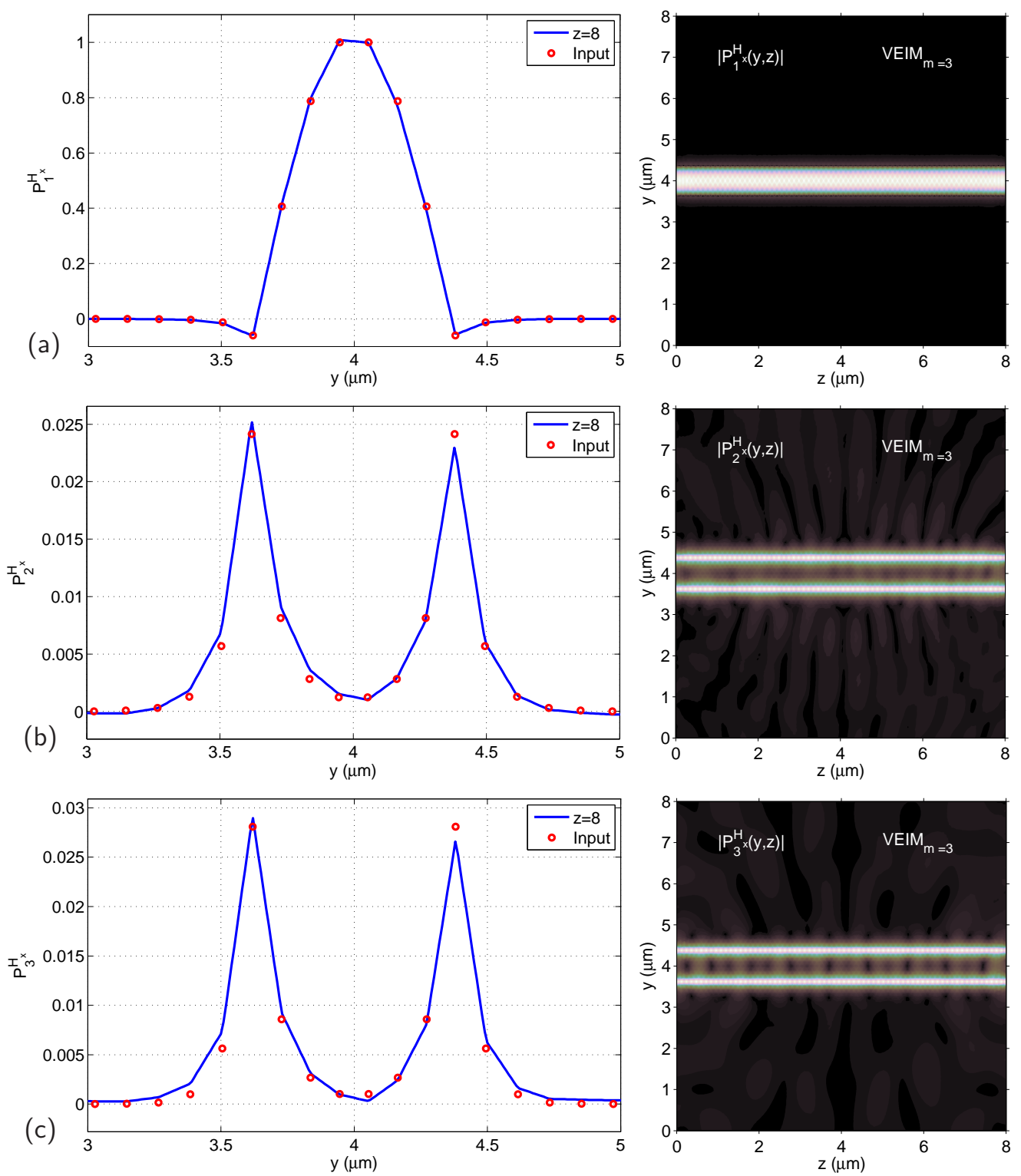

Figure 5.6: Input mode profiles and fields at the right-hand side of the window $(z=8 \mu m)$ (left column), and a top view of $\left|P_{j}^{H x}\right|$ (right column). The mesh size inside the waveguide is approximately 109nm. For the sake of visibility of the graphs, the right-hand pictures are all normalized to their own maximum value. 

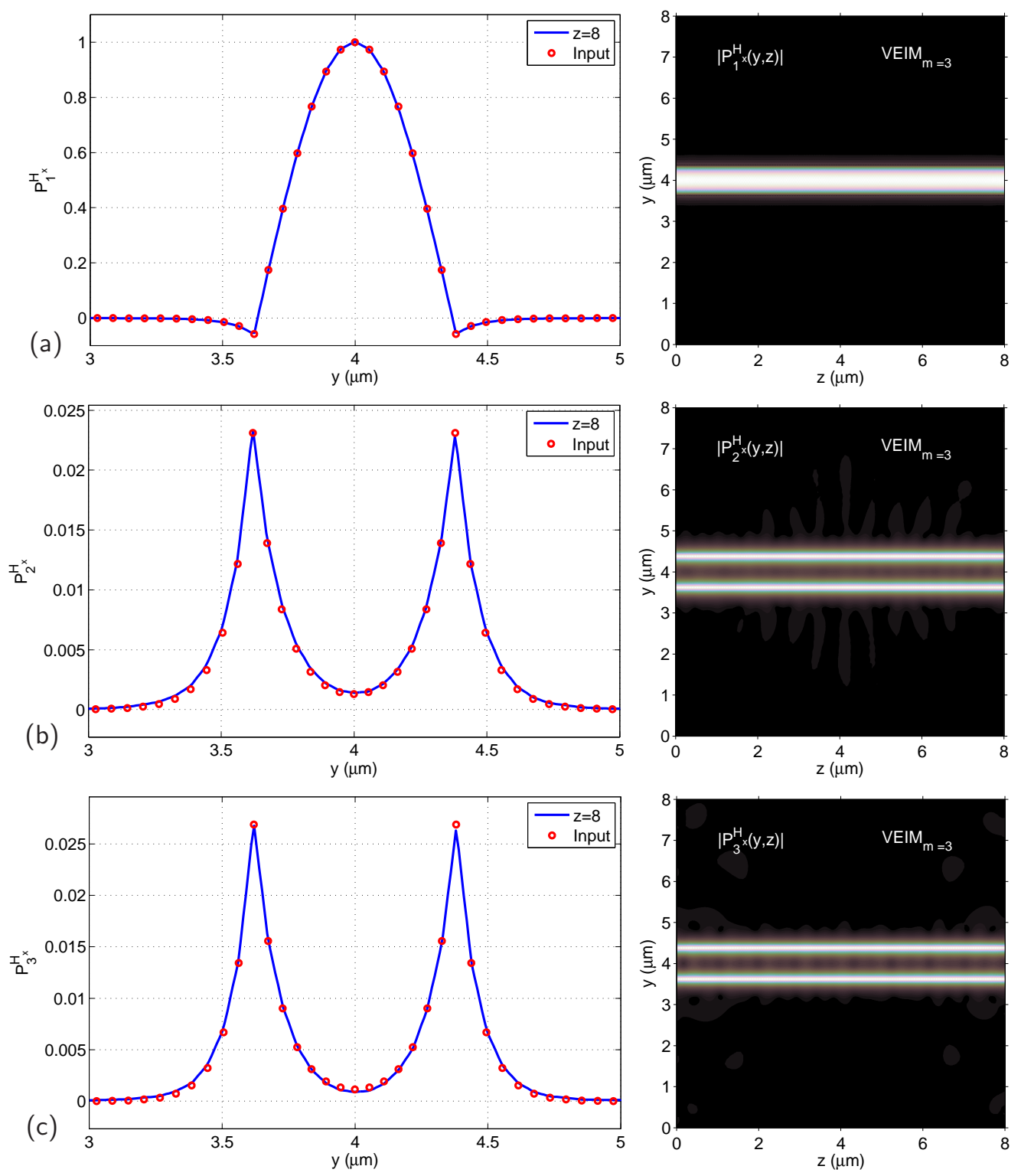

Figure 5.7: Input mode profiles and fields at the right-hand side of the window $(z=8 \mu m)$ (left column), and a top view of $\left|P_{j}^{H x}\right|$ (right column). The mesh size inside the waveguide is approximately $54 \mathrm{~nm}$. For the sake of visibility of the graphs, the right-hand pictures are all normalized to their own maximum value. 
For the two coarsest meshes, plots of the exterior mode and the solution on the right-hand boundary are shown in Figure 5.5, together with plots of the absolute value of $P_{1}^{H x}$ on the whole interior.

One can see that the field of the finer mesh is much smoother inside the interior, and the field on the right-hand boundary is near indistinguishable from the input mode - while the coarser mesh shows artefacts in the 2D view, and the output field is not as similar to the input mode. The asymmetry that is visible in the fields is caused by the mesh; COMSOL does not create a symmetric mesh.

When using three TE modes in the expansion, all of them contribute to the input mode, and they should thus all propagate through the waveguide with the same propagation constant, while keeping their shape. Figure 5.6 and Figure 5.7 again show the shape of the coefficients of all three slab modes $P_{j}^{H x}$ for the input mode and at the right-hand boundary of the calculation window, as well as a graph of their absolute values in the whole window, for two mesh sizes.

Also with three modes in the expansion, the mode propagates with approximately the same effective index as with one mode; more vertical profiles would be needed to obtain results closer to the reference effective index. All three slab modes propagate with propagation constants that are well within $1 \%$ of each other. Similar to what was seen with one mode in the expansion, the mode field deforms somewhat during propagation for the coarser mesh; on the finer mesh, the fields at the right-hand boundary are virtually identical to the mode that was launched from the left-hand boundary.

\subsubsection{Photonic crystal slab waveguide}

In this section we calculate the transmission spectrum of the structure shown in Figure 5.8. First with only a single mode in the expansion, and then with more modes to account for radiation losses. The results are compared with 3D FDTD [23] simulations. 


\section{1 mode in expansion}

One reason why it is interesting to apply our method to this structure is the fact that no guided mode exists in the vertical slices, where holes are located. So to apply 'standard' EIM one has to guess the effective refractive index of those regions: should it be the refractive index of the $\mathrm{SiO}_{2}$ substrate, or of air, or something in between? VEIM, with one mode in the expansion, uniquely defines these numbers.
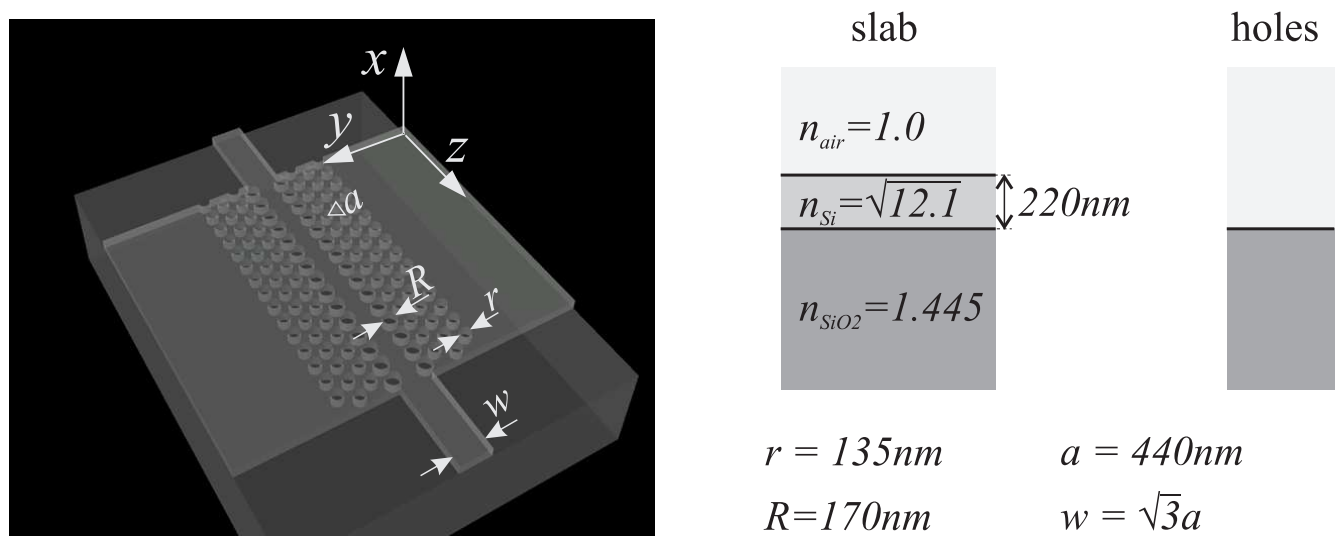

Figure 5.8: Photonic crystal slab waveguide. The structure is composed of a $220 \mathrm{~nm}$ thick $\mathrm{Si}\left(n_{\mathrm{Si}}=\sqrt{12.1}\right)$ layer on top of a SiO $\mathrm{S}_{2}\left(n_{\mathrm{SiO}_{2}}=1.445\right)$ substrate with air $\left(n_{\text {air }}=1.0\right)$ around. The waveguide and holes are defined by etching fully through the Si layer. The triangular lattice photonic crystal with lattice constant $a=440 \mathrm{~nm}$ consists of circular holes with radius $135 \mathrm{~nm}$; the input and output waveguides are $\sqrt{3} a \approx 762 \mathrm{~nm}$ wide. A defect waveguide is created by removing a row of holes and enlarging the first row of holes on either side to a radius of $170 \mathrm{~nm}$. In total, there are four rows of holes on either side of the defect waveguide. The calculation window $y \in[0,8] \mu m, z \in[0,10.635] \mu m$ is discretized into 266880 linear triangular finite elements.

Since the slab mode we will use in the expansion is a guided mode, we do not use PMLs in this simulation. Furthermore, instead of applying finite elements in the $x$-direction, the slab modes are calculated by a semianalytical mode solver, which also provides semi-analytical overlap integrals [65]. 


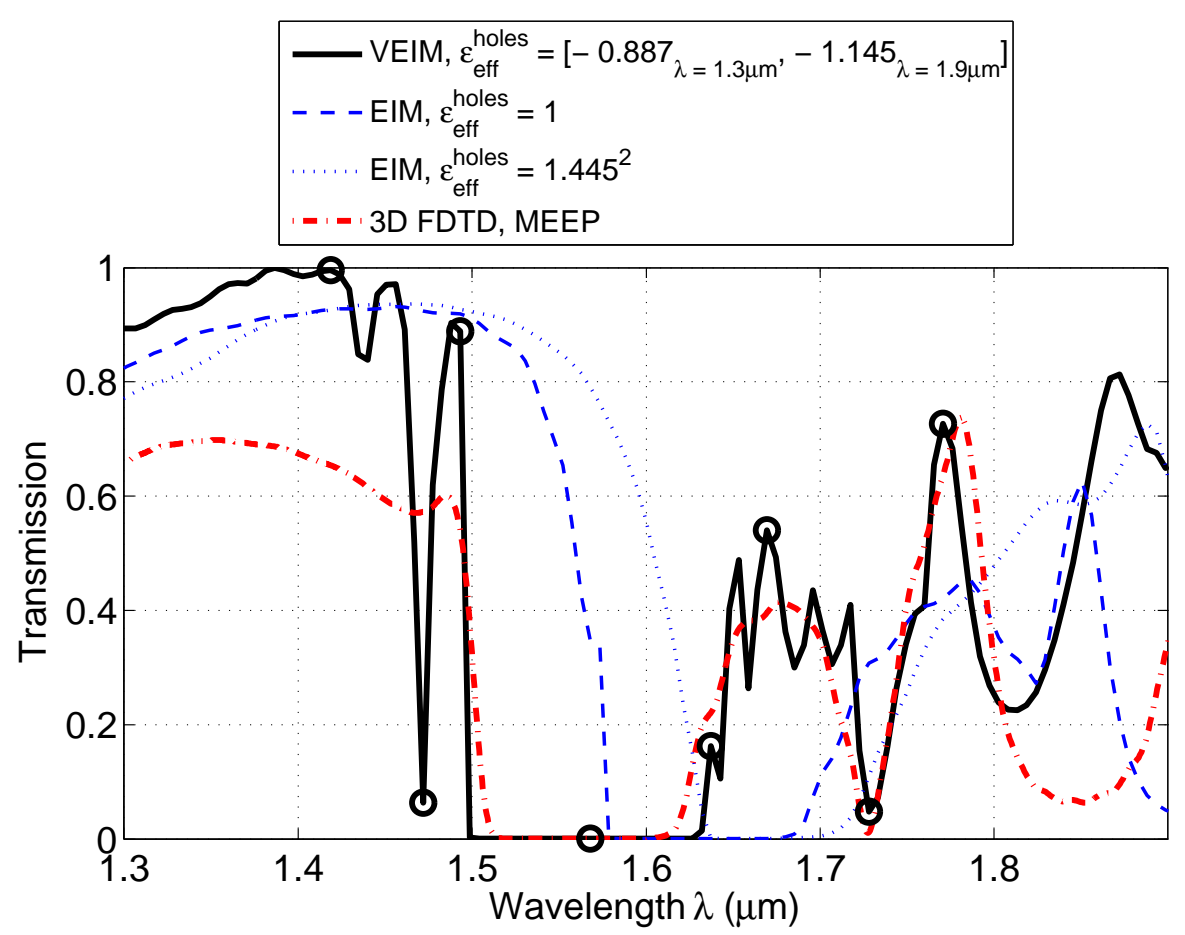

Figure 5.9: Transmission spectrum of the photonic crystal waveguide shown in Figure 5.8. The curves labeled 'EIM' use the Effective Index Method with the given effective permittivity for the hole regions; the corresponding values in the equations for VEIM vary as a function of the wavelength between the given values. The reference FDTD data is generated using [23].

As shown in Figure 5.9, the VEIM predictions of the location of the stopband and the general spectral features are reasonably close to the 3D FDTD reference results, while the 'conventional' EIM data, using either the cladding (1.0) or substrate refractive indices (1.445) as effective values for the hole regions, are much further off. Note that the effective permittivity (5.8.52) can very well turn out to be negative, as it happens to be in the present example. Inside the hole regions the effective permittivity varies between $-0.887 @ \lambda=1.3 \mu \mathrm{m}$ and $-1.145_{@ \lambda=1.9 \mu \mathrm{m}}$. So in all air regions in Figure 5.10 the field $P^{H x}$ decays exponentially. This figure shows plots of $\left|P^{H x}\right|$ at the wavelengths denoted by the circles in the spectrum of Figure 5.9.

By combining the values of $P^{H x}(y, z)$ with the slab mode field for $H_{x}$, the three-dimensional (semi-)vectorial field may be reconstructed, as demonstrated in Table 5.1. 

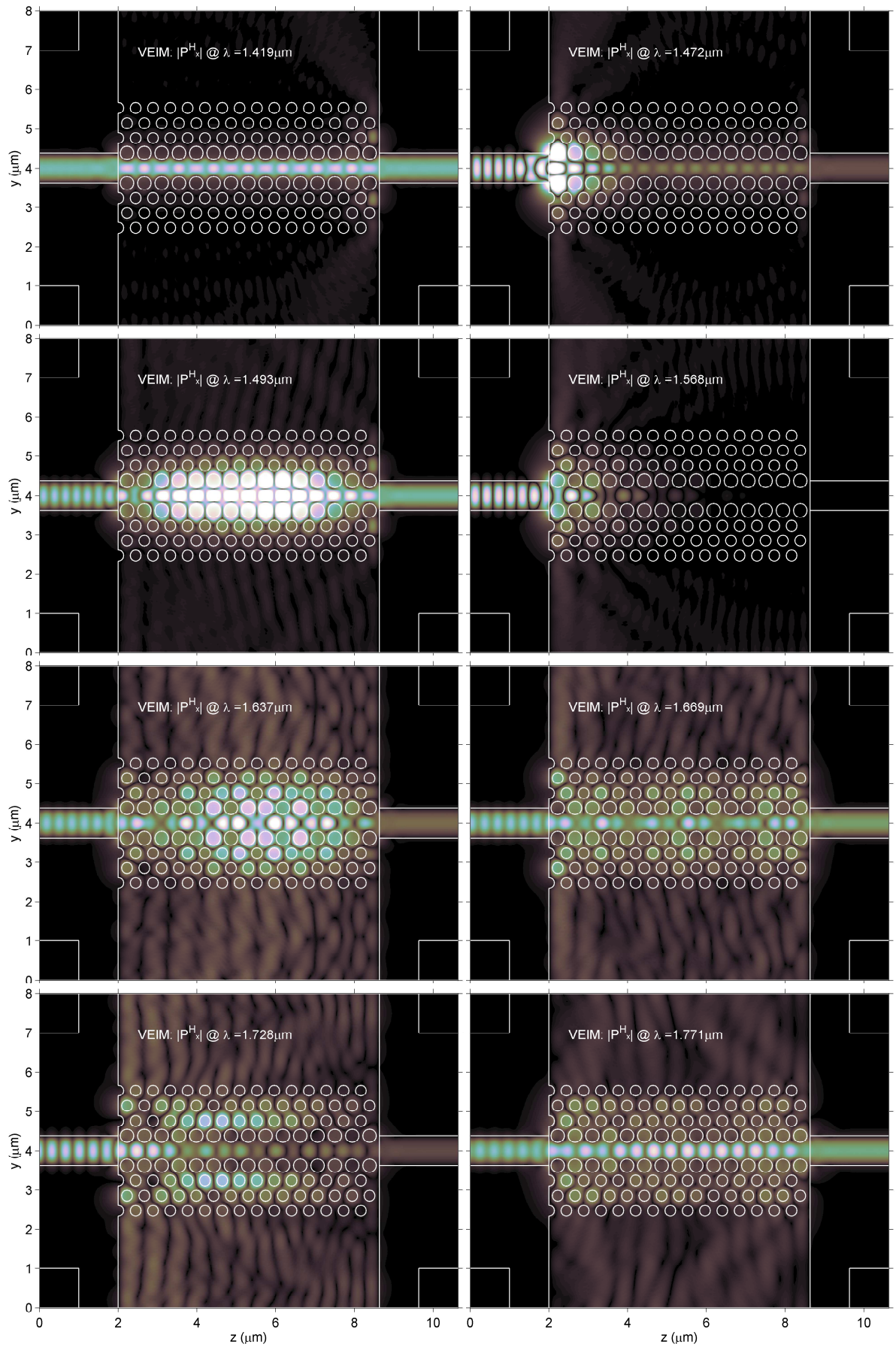

Figure 5.10: Light propagation through the photonic crystal slab waveguide of Figure 5.8 at several vacuum wavelengths; absolute value of $P^{H x}$. 

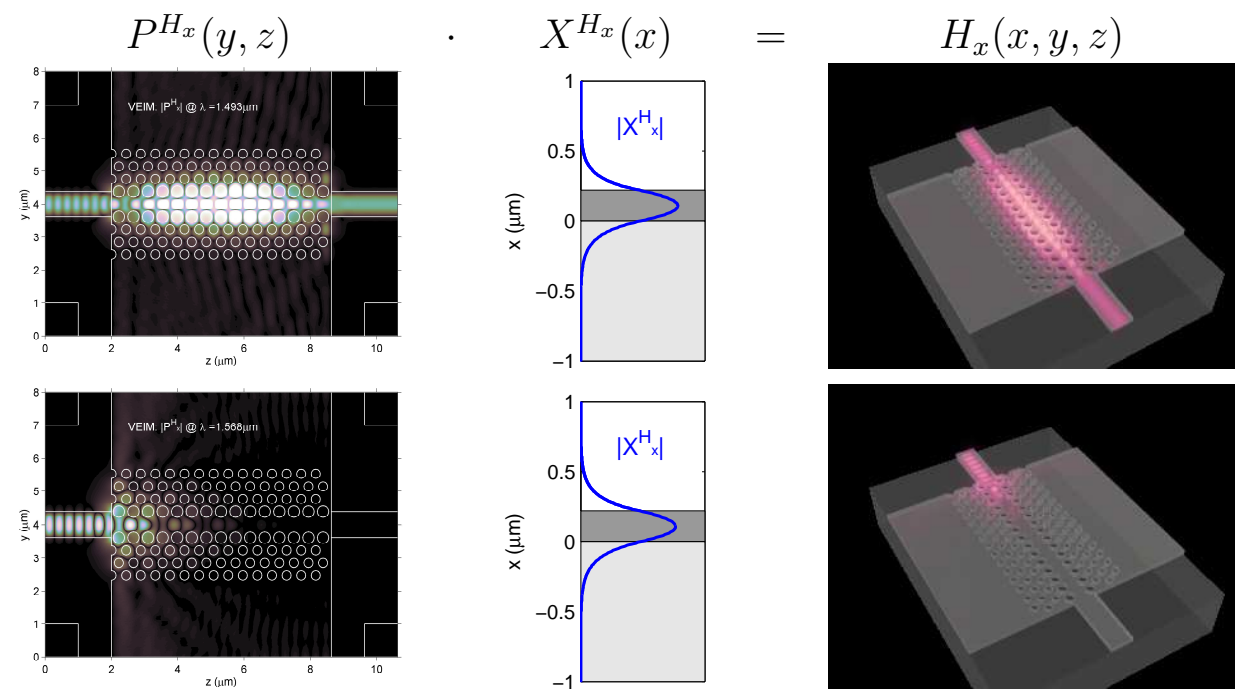

Table 5.1: Reconstruction of the 3D field profile at two wavelengths.

\section{TE modes in expansion}

The slab waveguide, in which we calculate the slab modes used in the expansion, does not support more than one guided TE mode. Therefore, any additional modes that we add to the expansion will be modes that are not guided and that should, thus, be leaky. In the calculations of the slab modes we therefore utilize $0.5 \mu \mathrm{m}$ thick PMLs at the boundaries of the computational domain in the $x$-direction, in exactly the same way as in Chapter 4 . $\sigma_{\max }$, as defined in the same chapter, is taken to be 1.2. Also the same finite element slab mode solver is applied, taking 272 linear elements on the computational window $x \in[-3,3.2] \mu \mathrm{m}$. We take the three highest order modes into the expansion, and calculate on the same mesh as before. We add $1 \times 1 \mu \mathrm{m}$ squares of PML with strength $\sigma=0.5$ in all four corners of the window in the $(y, z)$-plane, and extending into the exterior, to absorb radiation hitting the corners. Figure 5.11 shows how the spectrum of the device is changed by the addition of modes.

The addition of the extra modes does not appear to shift the spectrum much, but their main effect is to decrease the transmission due to light being coupled into the higher order slab modes, which may radiate away and be absorbed by the PMLs. Figure 5.12 shows the coefficients of all three modes in the $y-z$ plane. 

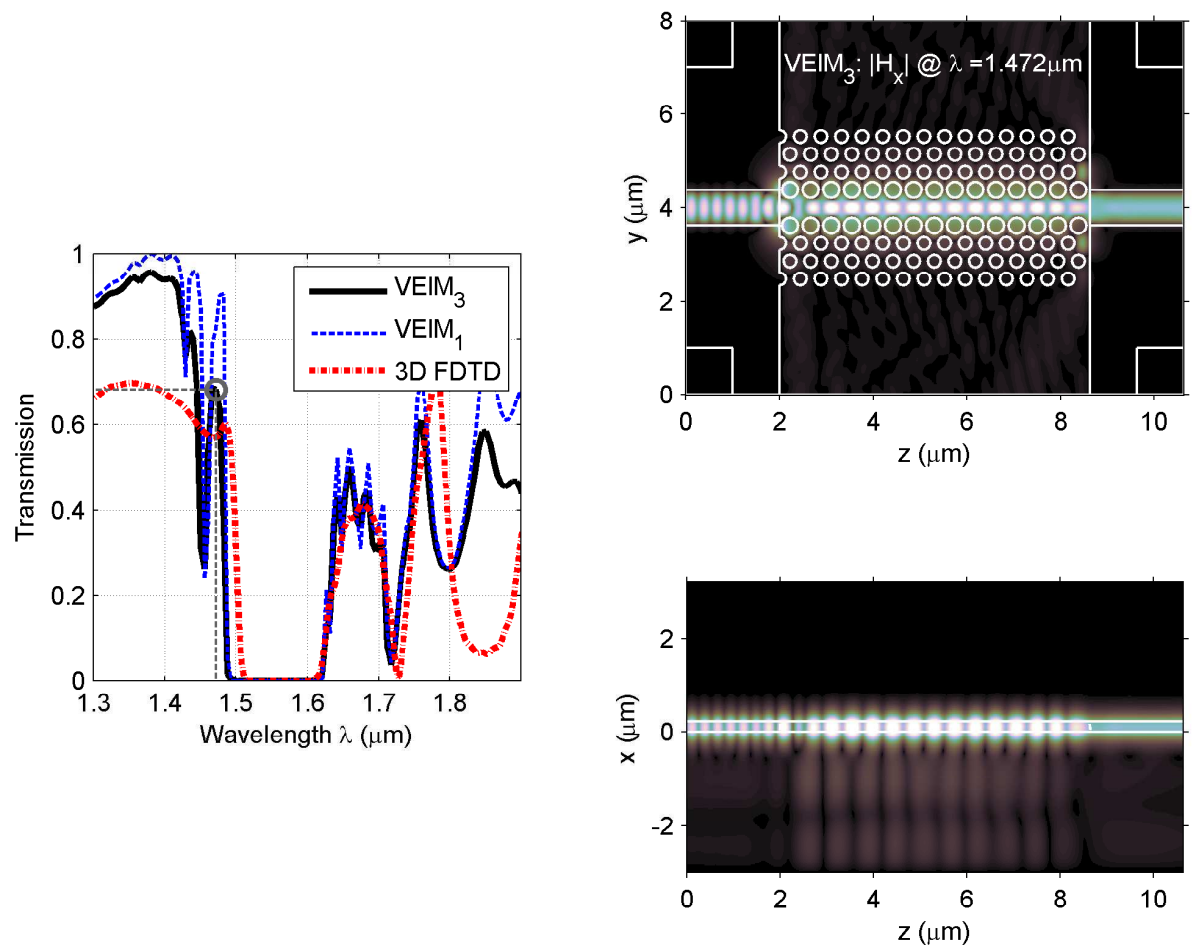

Figure 5.11: Left: Calculated spectrum with 1 mode (VEIM $)_{1}$ or 3 modes $\left(V E I M_{3}\right)$ in the expansion - both using slab modes calculated with 272 finite elements in the expansion. Note that the spectrum is shifted slightly with respect to Figure 5.9 due to the difference between these numerically calculated slab modes and the semi-analytic modes used in Figure 5.9. Right: Absolute value of the $H_{x}$ field in two cross-sections; top: $y$-z plane in the center of the silicon layer; bottom: $x-z$ plane in the center of the waveguide
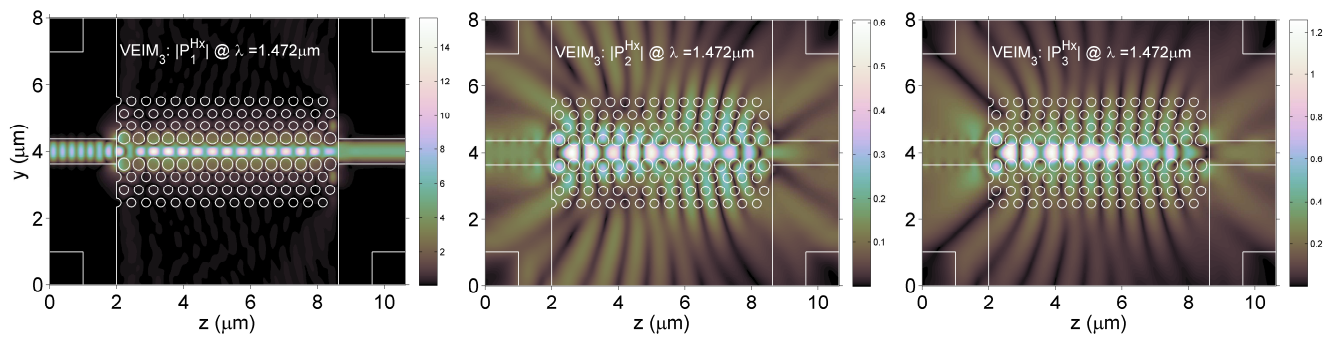

Figure 5.12: Absolute value of the coefficients of the three modes involved in the results shown in Figure 5.11 . 


\subsection{Conclusions}

By means of an expansion over TE and TM slab modes in one direction, the dimensionality of vectorial 3D scattering problems has been reduced. A Finite Element Method solves the resulting system of 2D equations; the boundaries of the calculation window are made to be transparent to outgoing light using modified Transparent Influx Boundary Conditions. PMLs at the top and bottom of the window absorb vertically scattered radiation.

The method is validated by means of a straight silicon-on-insulator waveguide example; subsequently, it is applied to a photonic crystal waveguide. We demonstrated that in case of the photonic crystal waveguide the current method with one mode in the expansion predicts the bandgap and other spectral features much more precisely than a standard Effective Index Method, at more or less the same computational cost. More modes in the expansion allow radiation loss to be taken into account.

While the current method is in principle suitable for general, slab-like non-uniform structures, our approach could be applied in a different way to structures that mainly consist of sections that are invariant in the $z$ direction. The field at each $z$-position can be expanded in the vector components of $2 \mathrm{D}$ modes of one reference $(x, y)$-cross-section; the resulting set of coupled 1D equations could be solved in a similar way as described in Chapter 4. Such an algorithm would be comparable to a 3D Bidirectional Eigenmode Propagation (BEP) method, with the distinct advantages that modes only need to be calculated on one cross-section, and that the continuity conditions of the fields between sections would be satisfied automatically.

There may be yet another approach to the 3D scattering problem. In the results of the present method we observed that the system of partial differential equations of the reduced problem decouples in the reference slice. Thus, if we use a set of local slab modes in the expansion in each distinct region, the system of equations may be decoupled everywhere, except on the interfaces between regions. On those interfaces, continuity conditions couple the solutions in the two regions. Such largely decoupled systems should be cheap to solve; in the finite element method, the matrices will be much more sparse. Since the solution in each region is essentially just a superposition of rotated slab modes, such a method could be seen as an analogue of the Film Mode Matching mode solving method, where the solution on each uniform slice is also represented as a superposition of rotated slab modes, which couple at interfaces between slices. 


\section{Conclusions and recommendations}

\section{Concluding remarks}

During this work we developed and implemented global eigenmode expansion methods for a large variety of problems: scalar and vectorial mode solvers, as well as 2- and fully 3-D scattering problems. All methods are based on the expansion of the field along one spatial direction in the slab modes of some reference slice(s); a variational procedure is used to derive the equations in the other spatial direction(s). In the mode solvers, both finite element and semi-analytical techniques were used to solve the resulting equations; for the scattering problems, only finite element implementations have been created.

When searching for guided modes, the fields decay toward infinity, so no special boundary conditions are needed. However, in scattering problems light must be able to leave the calculation window unimpeded, and it needs to be possible to prescribe influx from outside the window. Therefore we developed advanced boundary conditions, which are a combination of Perfectly Matched Layers (PMLs) and Transparent Influx Boundary Conditions (TIBCs). PMLs allow the method to correctly model structures with radiation losses, whereas without them, this radiation would reflect off the hard boundary walls and disturb the results. However, while PMLs work very well as absorbers, prescribing influx is not trivial. Therefore, we took the concept of TIBCs, which allow radiation to leave the window while also allowing influx to be prescribed, and extended them to the present mode expansion method.

The scalar and vectorial mode solvers were implemented in our own $\mathrm{C}++$ 
programs. Results from comparisons with benchmark problems, including both piecewise constant channel waveguides and diffused waveguides, show that the present method can provide very accurate results even with only a few modes in the expansion, and that it converges to the benchmark results if the number of modes in the expansion is increased.

We implemented numerical techniques for scattering problems in COMSOL Multiphysics - a package that employs an advanced finite element method. An influx is defined in terms of amplitudes of incoming modes and implemented by modifying the standard boundary conditions of COMSOL. Since the method can be applied to a wide range of geometries, the current approach is a valuable option for fully vectorial 3D simulations. Results from the scattering solvers on several structures, like Bragg gratings, tapers, and photonic crystal slabs, have been compared to reference results obtained by Finite Difference Time Domain (FDTD). Also here, we find good results with a few modes in the expansion, while more modes make the results converge toward the reference results.

The examples have shown that, on the one hand, while the method with only one or a few modes in the expansion gives a rather crude approximation to the optical field, it still in most cases performs much better than the standard Effective Index approach, on the other hand it can be used for rigorous computations by expanding the field over multiple vertical slab modes.

\section{Outlook}

There are several topics that could be the subject of further work and extensions to the current programs.

One extension, which is especially relevant for plasmonic structures, is the ability to handle metals and other lossy materials. An advantage of frequency domain solvers compared to time domain solvers is the fact that the high dispersion that metals exhibit is automatically covered. Moreover, all expressions, i.e. the functional, overlap integrals etc. already apply to metallic structures.

The presented mode solvers can be extended to also deal with radiation loss (leaky modes) by e.g. surrounding the computational window with PMLs. This does, however, complicate the search for propagation constants, since they will lie in the complex plane and no longer on the real or imaginary axis. Furthermore, nonphysical modes whose power is localized in the 
PMLs may be erroneously found.

There is also a possibility to extend the current method to deal with anisotropic materials, since the functionals for vectorial fields are also valid in this case.

The present 3D scattering method can be extended to solve the band structure of photonic crystal slabs - where instead of fixing the Bloch vector and solving for the frequency, as is done on many current algorithms, one would fix the frequency and solve for Bloch vectors.

In the conclusions of Chapter 5, two alternative approaches to tackle the 3D scattering problem were identified. In one, an expansion in $2 \mathrm{D}$ modes would be used in what can be considered a 3D to 1D dimensionality reduction, as an alternative to 3D Bidirectional Eigenmode Propagation methods. In the other approach, the field in each distinct region would be expanded in local slab modes, which then only couple on interfaces between regions; this approach can be considered a 3D generalization of the wellknown Film Mode Matching mode solving method.

Another important issue is the accuracy of the current approximations. While in this thesis we did not do more analysis than just to observe the convergence of our results with respect to external benchmark results, it would be very useful if there would be an algorithm that would, for example, determine how many and which modes are needed in the expansion to obtain a given accuracy for a certain field parameter.

On the basis of these suggestions the flexibility and possible application domains of the present methods could be increased even further, leading to a broad set of tools to support the design of current and novel optical devices. 



\section{Bibliography}

[1] AMSIX. http://www.ams-ix.net/.

[2] T. M. Benson, R. J. Bozeat, and P. C. Kendall. Rigorous effective index method for semiconductor rib waveguides. IEE Proceedings, 139(1):67-70, 1992.

[3] J.-P. Berenger. A perfectly matched layer for the absorption of electromagnetic waves. Journal of Computational Physics, 114(2):185-200, 1994.

[4] P. Bienstman. Rigorous and efficient modelling of wavelength scale photonic components. PhD thesis, Ghent University, Belgium, 2001.

[5] P. Bienstman. Two-stage mode finder for waveguides with a 2D crosssection. Optical and Quantum Electronics, 36(1-3):5-14, 2004.

[6] P. Bienstman and R. Baets. Optical modelling of photonic crystals and VCSELs using eigenmode expansion and perfectly matched layers. Optical and Quantum Electronics, 33(4-5):327-341, 2001.

[7] P. Bienstman and R. Baets. Advanced boundary conditions for eigenmode expansion models. Optical and Quantum Electronics, 34(5/6):523-540, 2002. 
[8] W. J. Bock, I. Gannot, and S. Tanev, editors. Optical waveguide sensing and imaging. NATO Science for Peace and Security Series B: Physics and Biophysics. Springer, The Netherlands, 2006.

[9] W. Bogaerts, P. Bienstman, D. Taillaert, R. Baets, and D. de Zutter. Out-of-plane scattering in 1-D photonic crystal slabs. Optical and Quantum Electronics, 34(1-3):195-203, 2002.

[10] FieldDesigner by PhoeniX Software. http://www.phoenixbv.com/.

[11] OptoDesigner by PhoeniX Software. http://www.phoenixbv.com/.

[12] M. L. Calvo and V. Lakshminarayanan, editors. Optical waveguides: from theory to applied technologies. CRC Press, Taylor \& Francis Group, United States of America, 2007.

[13] W. Chew, J. Jin, and E. Michielssen. Complex coordinate stretching as a generalized absorbing boundary condition. Microwave and Optical Technology Letters, 15(6):363-369, 1997.

[14] W. C. Chew and W. H. Weedon. A 3D perfectly matched medium from modified Maxwell's equations with stretched coordinates. $\mathrm{Mi}$ crowave and Optical Technology Letters, 7(13):590-604, 1994.

[15] K. S. Chiang. Analysis of optical fibers by the effective-index method. Applied Optics, 25(3):348-354, 1986.

[16] K. S. Chiang. Review of numerical and approximate methods for the modal analysis of general optical dielectric waveguides. Optical and Quantum Electronics, 26(3):S113-S134, 1994.

[17] K. S. Chiang. Analysis of the effective-index method for the vector modes of rectangular-core dielectric waveguides. IEEE Transactions on Microwave Theory and Technology, 44(5):692-700, 1996.

[18] COMSOL. http://www.comsol.com.

[19] CorningSMF28e. http://www.corning.com/opticalfiber/ products/SMF-28e_XB_fiber.aspx.

[20] J. Crisp and B. Elliott, editors. Introduction to fiber optics. Newnes, UK, 2005. 
[21] M. Dems and W. Nakwaski. The modelling of high-contrast photonic crystal slabs using the novel extension of the effective index method. Optica Applicata, 36(1):51-56, 2006.

[22] FEMLAB. http://www.femlab.com.

[23] MEEP. Free finite-difference time-domain simulation software package developed at MIT. http://www.ab-initio.mit.edu/meep.

[24] L. Friedrich, P. Dannberg, C. Vächter, Th. Hennig, A. Bräuer, and W. Karthe. Directional coupler device using a three-dimensional waveguide structure. Optics Communications, 137(4-6):239-243, 1997.

[25] S. B. Gaál, H. J. W. M. Hoekstra, and P. V. Lambeck. Determining PML modes in 2D stratified media. Journal of Lightwave Technology, 21(1):293-298, 2003.

[26] D. F. G. Gallagher and T. P. Felici. Eigenmode expansion methods for simulation of optical propagation in photonics - pros and cons. In Proc. of Photonics West, San Jose, USA, 2003.

[27] R. Garg. Analytical and computational methods in electromagnetics. Artech House, USA, 2008.

[28] A. M. Goncharenko, V. A. Karpenko, V. N. Mogilevich, and A. B. Sotskii. Methods of approximate variable separation in the theory of weakly inhomogeneous optical waveguides (review). Journal of Applied Spectroscopy, 45(1):663-671, 1987.

[29] N. Gregersen and J. Mørk. An improved perfectly matched layer for the eigenmode expansion technique. Optical and Quantum Electronics, 40(11-12):957-966, 2008.

[30] E. van Groesen. Variational modelling for integrated optical devices. Seminar Mathematical Physics, University of Twente, Enschede, The Netherlands, 23 September 2002.

[31] E. W. C. van Groesen and J. Molenaar. Continuum modeling in the physical sciences. SIAM, USA, 2007. 
[32] G. R. Hadley and R. E. Smith. Full-vector waveguide modeling using an iterative finite-difference method with transparent boundary conditions. Journal of Lightwave Technology, 13(3):465-469, 1995.

[33] M. Hammer. Quadridirectional eigenmode expansion scheme for 2-D modeling of wave propagation in integrated optics. Optics Communication, 235(4-6):285-303, 2004.

[34] M. Hammer. Hybrid analytical/numerical coupled-mode modeling of guided wave devices. Journal of Lightwave Technology, 25(9):2287$2298,2007$.

[35] M. Hammer and O. V. Ivanova. On effective index approximations of photonic crystal slabs. In Proc. of the 13th Annual Symposium IEEE/LEOS Benelux Chapter, Enschede, The Netherlands, 2008.

[36] M. Hammer and O. V. Ivanova. Effective index approximations of photonic crystal slabs: a 2-to-1-D assessment. Optical and Quantum Electronics, 2009. (available online, DOI 10.1007/s11082-009-9349-3).

[37] W. L. C. Hopman, R. Stoffer, and R. M. de Ridder. High-resolution measurement of resonant wave patterns by perturbing the evanescent field using a nanosized probe in a transmission scanning near-field optical microscopy configuration. Journal of Lightwave Technology, 25(7):1811-1818, 2007.

[38] K. Iizuka. Elements of photonics. John Wiley \& Sons, USA, 2002.

[39] O. V. Ivanova, M. Hammer, R. Stoffer, and E. van Groesen. Variational effective index mode solver. In Proc. of the 11th Annual IEEE/LEOS Benelux Chapter Symposium, Eindhoven, The Netherlands, 2006.

[40] O. V. Ivanova, M. Hammer, R. Stoffer, and E. van Groesen. A variational mode expansion mode solver. Optical and Quantum Electronics, 39(10-11):849-864, 2007.

[41] O. V. Ivanova, R. Stoffer, and M. Hammer. Dimensionality reduction in computational photonics through variational mode expansion. In Proc. of MicroNano Conference, Ede, The Netherlands, 2008. 
[42] O. V. Ivanova, R. Stoffer, and M. Hammer. A dimensionality reduction technique for scattering problems in photonics. In Proc. of 1st International Workshop on Theoretical and Computational NanoPhotonics (TaCoNa-Photonics), Bad Honnef, Germany, 2008.

[43] O. V. Ivanova, R. Stoffer, and M. Hammer. A variational mode solver for optical waveguides based on quasi-analytical vectorial slab mode expansion. Optics Communication, 2009. (submitted).

[44] O. V. Ivanova, R. Stoffer, and M. Hammer. A dimensionality reduction technique for $2 \mathrm{D}$ scattering problems in photonics. Journal of Optics, 2010. (accepted).

[45] O. V. Ivanova, R. Stoffer, and M. Hammer. A dimensionality reduction technique for 3D vectorial scattering problems in photonics. Journal of Lightwave Technology, 2010. (in preparation).

[46] O. V. Ivanova, R. Stoffer, M. Hammer, and E. van Groesen. A variational vectorial mode solver. In Proc. of the 17th International Workshop on Optical Waveguide Theory and Numerical Modelling (OWTNM), Eindhoven, The Netherlands, 2008.

[47] O. V. Ivanova, R. Stoffer, M. Hammer, and E. van Groesen. A vectorial variational mode solver and its application to piecewise constant and diffused waveguides. In Proc. of the 12th International Conference on Mathematical Methods in Electromagnetic Theory (MMET), Odessa, Ukraine, 2008.

[48] O. V. Ivanova, R. Stoffer, L. Kauppinen, and M. Hammer. Variational effective index method for $3 \mathrm{D}$ vectorial scattering problems in photonics: TE polarization. In Proc. of Progress In Electromagnetics Research Symposium (PIERS), Moscow, Russia, 2009.

[49] S. Janz, J. Čtyroký, and S. Tanev, editors. Frontiers in planar lightwave circuit technology. Springer, Netherlands, 2006. Chapter: Integrated optics design: software and diversified applications.

[50] J. Jin. The Finite Element Method in Electromagnetics. Wiley-IEEE Press, New York, 2002. 
[51] J. D. Joannopoulos, S. G. Johnson, J. N. Winn, and R. D. Meade. Photonic crystals: molding the flow of light. Princeton University Press, USA, 2008.

[52] J. van Kan, G. Segal, and F. Vermolen. Numerical methods in scientific computing. VSSD, Delft, The Netherlands, 2005.

[53] K. Kawano and T. Kitoh. Introduction to optical waveguide analysis: solving Maxwell's equations and the Schrödinger equation. John Wiley \& Sons, USA, 2001.

[54] S. A. Kemme. Microoptics and nanooptics fabrication. CRC Press, Taylor \& Francis Group, USA, 2010.

[55] M. Kitsara. Silicon micromachined optical sensors for chemical/biological assays. PhD thesis, University of Ioannina, Greece, 2009.

[56] E. J. Klein. Densely integrated microring-resonator based components for fiber-to-the-home applications. $\mathrm{PhD}$ thesis, University of Twente, The Netherlands, 2007.

[57] E. E. Kriezis and A. G. Papagiannakis. A three-dimensional full vectorial Beam Propagation Method for $z$-dependent structures. IEEE Journal of Quantum Electronics, 33(5):883-890, 1997.

[58] LAPACK. http://www.netlib.org/lapack/.

[59] G. Lifante. Integrated photonics: fundamentals. John Wiley \& Sons, England, 2003.

[60] M. Lohmeyer. Guided waves in rectangular integrated magnetooptic devices. PhD thesis, University of Osnabrück, Germany, 1999.

[61] M. Lohmeyer. Mode expansion modeling of rectangular integrated optical microresonators. Optical and Quantum Electronics, 34(5):541$557,2002$.

[62] M. Lohmeyer and R. Stoffer. Integrated optical cross strip polarizer concept. Optical and Quantum Electronics, 33(4/5):413-431, 2001. 
[63] Y. Y. Lu. Some techniques for computing wave propagation in optical waveguides. Communications in Computational Physics, 1(6):1056$1075,2006$.

[64] R. März. Integrated optics - design and modelling. Artech house, Norwood, 1995.

[65] M. Hammer. METRIC Mode expansion tools for 2D rectangular integrated optical circuits. http://www.math.utwente.nl/ hammerm/ Metric/.

[66] F. Morichetti, A. Melloni, M. Martinelli, R. G. Heideman, A. Leinse, D. H. Geuzebroek, and A. Borreman. Box-shaped dielectric waveguides: a new concept in integrated optics? Journal of Lightwave Technology, 25(9):2579-2589, 2007.

[67] W. C. Ng and M. S. Stern. Analysis of multiple-rib waveguide structures by the discrete-spectral-index method. IEE Proceedings, 145(6):365-371, 1998.

[68] J. B. Nicolau and E. van Groesen. Hybrid analytic-numeric method for light through a bounded planar dielectric domain. Journal of Nonlinear Optical Physics and Materials, 14(2):161-176, 2005.

[69] M. Noureddine, T. M. Benson, C. J. Smartt, and P. C. Kendall. Complex general Fourier variational method applied to the analysis of semiconductor lasers. IEE Proceedings, 144(4):241-245, 1997.

[70] S. S. A. Obayya, N. Somasiri, B. M. A. Rahman, and K. T. V. Grattan. Full vectorial finite element modeling of novel polarization rotators. Optical and Quantum Electronics, 35(4-5):297-312, 2003.

[71] K. Okamoto. Fundamentals of optical waveguides. Academic Press, London, UK, 2006.

[72] OptiSense. http://www.optisense.nl/.

[73] W. Park and J.-B. Lee. Mechanically tunable photonic crystal structure. Applied Physics Letters, 85(21):4845-4847, 2004. 
[74] M. Paulus and O. J. F. Martin. A fully vectorial technique for scattering and propagation in three-dimensional stratified photonic structures. Optical and Quantum Electronics, 33(4-5):315-325, 2001.

[75] L. Pavesi and D. J. Lockwood, editors. Silicon photonics. Springer, Germany, 2004.

[76] S. T. Peng and A. A. Oliner. Guidance and leakage properties of a class of open dielectric waveguides: part I - mathematical formulations. IEEE Transactions on Microwave Theory and Techniques, 29(9):843$855,1981$.

[77] R. Pregla. Analysis of electromagnetic waves: the Method of Lines. John Wiley \& Sons, England, 2008.

[78] M. Qiu. Effective index method for heterostructure-slab-waveguidebased two-dimensional photonic crystals. Applied Physics Letters, 81(7):1163-1165, 2002.

[79] M. Qiu, K. Azizi, A. Karlsson, M. Swillo, and B. Jaskorzynska. Numerical studies of mode gaps and coupling efficiency for line-defect waveguides in two dimensional photonic crystals. Physical Review B, 64:155113-1-5, 2001.

[80] M. J. Robertson, P. C. Kendall, C. Ritchie, P. McIlroy, and M. J. Adams. The weighted index method: a new technique for analysing planar optical waveguides. Journal of Lightwave Technology, 7(12):2105-2111, 1989.

[81] K. Sakoda. Optical properties of photonic crystals. Springer, Germany, 2005.

[82] R. Scarmozzino, A. Gopinath, and R. Pregla. Numerical techniques for modeling guided-wave photonic devices. IEEE Journal of Selected Topics in Quantum Electronics, 6(1):150-162, 2000.

[83] J. M. Senior, editor. Optical fiber communications: principles and practice. Prentice Hall International Inc., UK, 1993.

[84] A. Sharma. Analysis of integrated optical waveguides: variational method and effective-index method with built-in perturbation correction. Journal of Optical Society of America A, 18(6):1383-1387, 2001. 
[85] A. Sharma and P. Bindal. An accurate variational analysis of single-mode diffused waveguides. Optical and Quantum Electronics, 24(12):1359-1371, 1992.

[86] S. Shi, C. Chen, and D. W. Prather. Revised plane wave method for dispersive material and its application to band structure calculations of photonic crystal slabs. Applied Physics Letters, 86:043104-1-3, 2005.

[87] C. T. Shih, Z. W. Zeng, Y. C. Chang, Y.-J. Lee, and S. Chao. Optical design of bent rib waveguide with MOS cross-section. Optical Review, 16(4):413-415, 2009.

[88] A. W. Snyder and J. D. Love. Optical waveguide theory. Kluwer Academic Publishers, London, Great Britain, 2000.

[89] A. Sopaheluwakan. Characterization and simulation of localized states in optical structures. PhD thesis, University of Twente, The Netherlands, 2006.

[90] R. Stoffer. Uni- and omnidirectional simulation tools for integrated optics. PhD thesis, University of Twente, The Netherlands, 2001.

[91] R. Stoffer, O. V. Ivanova, M. Hammer, and H. J. W. M. Hoekstra. A guided mode view on Near-field Scanning Optical Microscopy measurements of optical magnetic fields with slit probes. In Proc. of the 19th International Workshop on Optical Waveguide Theory and Numerical Modelling (OWTNM), Cambridge, UK, 2010. (submitted).

[92] R. Stoffer, O. V. Ivanova, and T. Korthorst. Smart algorithms and smart design tools. Mikroniek, 49(5):21-25, 2009.

[93] R. Stoffer, A. Sopaheluwakan, M. Hammer, and E. van Groesen. Helmholtz solver with transparent influx boundary conditions and nonuniform exterior. In Proc. of 16th International Workshop on Optical Waveguide Theory and Numerical Modelling, Copenhagen, Denmark, 2007. book of abstracts 3 .

[94] R. Stoffer, A. Sopaheluwakan, M. Hammer, and E. van Groesen. A combination of Dirichlet to Neumann operators and Perfectly 
Matched Layers as boundary conditions for optical finite element simulations. In Proc. of 12th International Conference on Mathematical Methods in Electromagnetic Theory, Odesa, Ukraine, 2008.

[95] A. S. Sudbø. Film mode matching: a versatile numerical method for vector mode field calculations in dielectric waveguides. Pure and Applied Optics, 2(3):211-233, 1993.

[96] G. Sztefka and H. P. Nolting. Bidirectional eigenmode propagation for large refractive index steps. IEEE Photonics Technology Letters, 5(5):554-557, 1993.

[97] A. Taflove and S. C. Hagness. Computational electrodynamics: the Finite-Difference Time-Domain method. Artech House, Norwood, 2005.

[98] F. Tisseur and K. Meerbergen. The quadratic eigenvalue problem. SIAM Review, 43(2):235-286, 2001.

[99] I. Tsukerman. Computational methods for nanoscale applications: particles, plasmons and waves. Springer, New York, 2008.

[100] J. J. G. M. van der Tol and N. H. G. Baken. Correction to the Effective Index Method for rectangular dielectric waveguides. Electronics Letters, 24(4):207-208, 1988.

[101] C. Vassallo. Optical waveguide concepts. Elsevier, Amsterdam, 1991.

[102] C. Vassallo. 1993-1995 optical mode solvers. Optical and Quantum Electronics, 29(2):95-114, 1997.

[103] C. Vassallo and Y. H. Wang. A new semirigorous analysis of rib waveguides. Journal of Lightwave Technology, 8(1):56-65, 1990.

[104] J. Čtyroký. Photonic bandgap structures in planar waveguides. Journal of the Optical Society of America A, 18(2):435-441, 2001.

[105] J. Čtyroký. Improved bidirectional-mode expansion propagation algorithm based on Fourier series. Journal of Lightwave Technology, 25(9):2321-2330, 2007. 
[106] J. Čtyroký, S. Helfert, R. Pregla, P. Bienstman, R. Baets, R. de Ridder, R. Stoffer, G. Klaasse, J. Petrcek, P. Lalanne, J.-P. Hugonin, and R. M. De La Rue. Bragg waveguide grating as a 1D photonic band gap structure: COST 268 modelling task. Optical and Quantum Electronics, 34:455-470, 2002.

[107] K. van de Velde, H. Thienpont, and R. van Geen. Extending the effecive index method for arbitrary shaped inhomogeneous optical waveguides. Journal of Lightwave Technology, 6(6):1153-1159, 1988.

[108] J. Willems, J. Haes, and R. Baets. The bidirectional mode expansion method for two-dimensional waveguides: the TM case. Optical and Quantum Electronics, 27:995-1007, 1995.

[109] J. Witzens, M. Lončar, and A. Scherer. Self-collimation in planar photonic crystals. IEEE Journal of Selected Topics in Quantum Electronics, 8(6):1246-1257, 2002.

[110] L. Yang, J. Motohisa, and T. Fukui. Suggested procedure for the use of the effective-index method for high-index-contrast photonic crystal slabs. Optical Engineering, 44(7):078002-1-078002-7, 2005.

[111] K. Yasumoto, editor. Electromagnetic theory and applications for photonic crystals. CRC Press, Taylor \& Francis Group, USA, 2006. 



\section{Summary}

This thesis describes a new set of methods for the simulation of light propagation in photonic structures - in other words, methods to solve Maxwell's equations in optical chips. Due to common fabrication technologies, the light in optical chips is made to mainly propagate along the directions parallel to the initial substrate. We use this knowledge to develop a formalism which expands the field in the direction perpendicular to the substrate in a set of basis fields. Typically these basis fields are modes that are supported by one or more vertical cross-sections. Given those basis fields, a variational method is applied to obtain the equations for the coefficients of the slab modes, or components thereof, in the plane of the substrate. In such a way, the dimensionality of the problem is reduced: Instead of a three (two) dimensional problem, a set of coupled two (one) dimensional problems need to be solved. Contrary to other well-known methods like the Effective Index Method or Mode Matching Methods, the same expansion is used everywhere in the structure - which ensures continuity of the relevant components.

Four important classes of problems are chosen, all of which are subjected to the treatment described above. These four problems are scalar mode solving, vectorial mode solving, 2D scattering, and 3D scattering problems.

If the refractive index contrast is low, implying that certain derivatives of the index can be neglected, a scalar mode equation can be derived. Solutions of this equation describe modes running along a waveguide with a certain propagation constant. Slab modes of one or more cross-sections are used 
to expand the field vertically. A semi-analytical solution method for the resulting set of $1 \mathrm{D}$ equations yields result that were compared with several other methods on various waveguide geometries. Somewhat remarkably, the comparison indicates that a reasonable accuracy in the computation of propagation constants can be achieved with rather few (sometimes: single) modes in the field expansion. No problem arises, if one of the constituting slices is below cut-off.

For the vectorial mode problem, the full Maxwell's equations are solved; all six electromagnetic vector field components are taken into account. Mode expansion in the vertical direction is combined with a variational formalism to obtain the equations in the horizontal direction, which are solved by means of either semi-analytic methods, or, for more complicated geometries, a finite element method. The method gives rather accurate estimates of the propagation constants, sometimes even with only a few terms in the expansion. The vectorial mode solver is applied to several structures and compared to reference results, and shows competitive performance. It turns out that if only one mode is used in the expansion, the method can be seen as an improved Effective Index Method, in which the coefficients are uniquely defined everywhere, even if no guided modes exist locally. While in the Film Mode Matching method, rotated modes of each slice are used to locally expand the field, our vectorial mode solver uses only one set of modes everywhere. We show that in the reference slice, where the 1D modes are calculated, we predict exactly the same rotations as the Film Mode Matching method uses. In the reference slice the total field profile is a superposition of these rotated 1D TE and TM modes; in other slices, however, the components of all the 1D modes mix.

In 2D scattering problems, the Maxwell's equations reduce to a TE or TM Helmholtz equation. In order to allow light to leave the structure vertically, we apply layers of artificial absorbing material, so-called Perfectly Matched Layers, at the top and bottom of the calculation window. The field is then expanded in slab modes of one or more reference slices. The resulting system of $1 \mathrm{D}$ differential equations is solved by means of a Finite Element Method. At the left and right edges of the computational window, boundary conditions are set up that allow influx to be prescribed, while allowing outgoing radiation to freely cross the boundary. The method is applied to grating and taper structures. As in a standard Effective Index Method (though with less heuristics involved), rough approximations can be obtained through expansions with one or only very few terms, at very 
low computational cost. In an intermediate regime already quite acceptable results emerge with only a very moderate number of expansion terms, as shown in some of our examples. Our calculations with a larger number of basis modes yield converged results, just as the established Bidirectional Eigenmode Propagation (BEP) schemes. Contrary to the BEP method, however, our solutions are continuous everywhere.

Finally, the 3D scattering problem involves solving the full vectorial Maxwell's equations. Again, the solution is globally expanded in vertical slab modes, and the equations in the other two directions are solved using a Finite Element Method. The boundaries of the computational window are made to be transparent for outgoing radiation by means of Transparent Influx Boundary Conditions, which utilize an expansion of the field of the exterior in modes, calculated by the algorithm of the $2 \mathrm{D}$ vectorial mode solver described above. Simulations on a straight dielectric waveguide give a first validation of the method, and results on a photonic crystal slab waveguide show that we can predict the location of the bandgap and other spectral features much more precisely than any 'standard' Effective Index Method. 



\section{Samenvatting}

Dit proefschrift beschrijft een aantal nieuwe methodes voor het simuleren van de voortplanting van licht in fotonische strukturen - in andere woorden, methodes voor het oplossen van de Maxwellvergelijkingen in optische chips. De gebruikelijke fabricage-technologie zorgt ervoor dat het licht in optische chips zich voornamelijk voortbeweegt in richtingen parallel aan het substraat. Wij gebruiken deze kennis om een formalisme te ontwikkelen dat het veld in de richting loodrecht op op het substraat in een set basisvelden ontwikkelt. Deze basisvelden zijn typisch golfgeleidermodes van één of meer vertikale doorsnedes, zogenaamde 'slab modes'. Gegeven die basisvelden, wordt een variationele methode toegepast om de vergelijkingen voor de coefficiënten van de slab modes, of van hun vectorcomponenten, in het vlak van het substraat te verkrijgen. Op zo'n manier wordt de dimensionaliteit van het probleem gereduceerd: In plaats van een drie-(twee-)dimensionaal probleem moet een set van gekoppelde twee-(één-)dimensionale problemen worden opgelost. In tegenstelling tot andere bekende methodes zoals de Effectieve Index Methode of Mode Matching Methodes, wordt dezelfde ontwikkeling overal in de struktuur gebruikt - wat zorg draagt voor de continuïteit van de relevante componenten.

Er zijn vier belangrijke klasses problemen gekozen, die alle aan de behandeling zoals hierboven besproken zijn blootgesteld. Deze vier problemen zijn het bepalen van scalaire modes, het bepalen van vectoriële modes, en $2 \mathrm{D}$ en $3 \mathrm{D}$ verstrooiingsproblemen.

Wanneer het brekingsindexcontrast laag is, wat impliceert dat bepaalde 
afgeleiden van de index verwaarloosd kunnen worden, kan een scalaire modevergelijking worden afgeleid. Oplossingen van deze vergelijking beschrijven modes die zich door een golfgeleider voortplanten met een bepaalde propagatieconstante. Slab modes van één of meer doorsnedes worden gebruikt om het veld vertikaal in te ontwikkelen. Een semi-analytische oplossingsmethode voor de resulterende set $1 \mathrm{D}$ vergelijkingen levert resultaten die vergeleken zijn met meerdere andere methodes, op verschillende golfgeleidergeometrieën. Enigszins opmerkelijk geeft de vergelijking aan dat er al een redelijke nauwkeurigheid bereikt kan worden in de berekening van propagatieconstantes met nogal weinig (soms: slechts één) modes in de veldontwikkeling. Er ontstaat geen probleem als één van de doorsnedes geen geleide mode ondersteunt.

Voor het vectoriële mode-probleem worden de volledige Maxwellvergelijkingen opgelost; alle zes electromagnetische vectorveldcomponenten moeten meegenomen worden. Een ontwikkeling in modes in de vertikale richting wordt gecombineerd met een variationeel formalisme om de vergelijkingen in de horizontale richting te verkrijgen, welke opgelost worden met behulp van semi-analytische methodes, of, voor ingewikkeldere geometrieën, een Eindige Elementen Methode. De methode geeft tamelijk nauwkeurige benaderingen van de propagatieconstantes, soms zelfs met slechts een paar termen in de ontwikkeling. De vectoriële mode solver wordt toegepast op verscheidene strukturen en vergeleken met referentieresultaten, en zet concurerende prestaties neer. Het blijkt dat in het geval dat slechts één mode wordt gebruikt in de ontwikkeling, de methode gezien kan worden als een verbeterde Effective Index Methode, waarin de coefficiënten overal uniek gedefinieerd zijn, zals als er lokaal geen geleide modes bestaan. Waar in de Film Mode Matching methode geroteerde modes van iedere doorsnede worden gebruikt om het veld lokaal te ontwikkelen, gebruikt onze mode solver slechts één set modes in het hele domain. Wij laten zien dat we in de referentie-doorsnede, waat de 1D modes worden uitgerekend, exact dezelfde rotaties voorspellen als in de Film Mode Matching methode worden gebruikt. In de referentie-doorsnede is het totale veldprofiel een superpositie van die geroteerde 1D TE en TM modes; echter, in andere doorsnedes worden de componenten van alle 1D modes gemengd.

In 2D verstrooï̈nsproblemen reduceren de Maxwellvergelijkingen tot een TE of TM Helmholtzvergelijking. Om het mogelijk te maken dat licht de struktuur in vertikale richting verlaat, passen we lagen van kunstmatig absorberend materiaal, zogenaamde Perfectly Matched Layers, toe aan 
de boven- en onderkant van het rekenvenster. Het veld wordt dan ontwikkeld in slab modes van één of meer referentiedoorsnedes. Het resulterende systeem 1D differentiaalvergelijkingen wordt opgelost met behulp van een Eindige Elementen Methode. Aan de linker- en rechterrand van het rekenvenster worden randvoorwaarden geïmplementeerd die het mogelijk maken een inkomend veld voor te schrijven, terwijl uitgaande straling de rand vrijelijk kan passeren. De methode wordt toegepast op tralie- en taperstrukturen. Net als in een standaard Effectieve Index Method (maar met minder heuristiek) kunnen ruwe benaderingen worden behaald middels ontwikkelingen in slechts één of een paar termen, tegen zeer lage rekenkosten. In een tussenliggend gebied komen al behoorlijk acceptabele resultaten naar voren met slechts een matig aantal ontwikkelingstermen, zoals in sommige van onze voorbeelden te zien is. Onze berekeningen met een groter aantal basismodes leveren geconvergeerde resultaten, net als de gevestigde Bidirectionele Eigenmode Propagatie (BEP) schema's. In tegenstelling tot de BEP methode zijn onze oplossingen echter wel overal continu.

Voor het 3D verstrooï̈nsprobleem dienen de volledige vectoriële Maxwellvergelijkingen opgelost te worden. Wederom wordt de oplossing globaal ontwikkeld in vertikale slab modes, en de vergelijkingen in de andere twee richtingen worden opgelost met behulp van een Eindige Elementen Methode. De randen van het rekenvenster worden transparant gemaakt voor uitgaande straling door Transparante Influx Randvoorwaarden, die gebruik maken van een ontwikkeling van het veld in het buitengebied in modes, uitgerekend met behulp van het algoritme van onze $2 \mathrm{D}$ vectoriële mode solver. Simulaties aan een rechte diëlectrische golfgeleider geven een eerste validatie van de methode, en resultaten aan een fotonisch kristal slab golfgeleider laten zien dat we de plaats van de bandgap en andere spectrale eigenschappen veel nauwkeuriger kunnen voorspellen dan welke 'standaard' effectieve index method dan ook. 



\section{Acknowledgments}

First of all I would like to mention my promoter prof. dr. Brenny van Groesen who gave me this opportunity to pursue my $\mathrm{PhD}$ at the University of Twente. I would also like to express my gratitude to my daily supervisor dr. Manfred Hammer. I am indebted to him for his daily support, stimulating advises, patience in correcting my articles. During these years I have learned a lot from him.

Especially I express my sincere thanks to our secretaries Mariëlle Slotboom-Plekenpol, Carin Krijnen-Smid, Diana Dalenoord and Linda Wychgel-van Dalm, who were not only ready to help me in every aspect of my life in Twente, but are also very nice people who made my stay at University a lot more enjoyable. I have to mention also my officemates Davit Harutyunyan, Sander Rhebergen, Domokos Sármány, Remco Stoffer, Tim op't Root, Marcel Lourens, She Liam Lie, Pablo Tassi and Bob Peeters for making our office a charming place. I also thank my colleagues from AAMP and NACM groups: Ferenc Izsák, Wenny Kristina, Didit Adytia, Ivan Lakhturov, Milan Maksimović, Sid Visser, Mike Botchev, Briti Sundar Deb, Lilya Ghazaryan, Sanne Smit, Marieke Kool, Tito Julianto, David Lopez-Penha, Massoumeh Mohammadi, Julia Mikhal, Shavarsh Nurijanyan, Henk Sollie, Anthony Thornton, Thomas Weinhart, Antonios Zagaris, Joris van den Berg, Fedderik van der Bos and Chris Klaij for being good colleagues and interesting company.

My distinct recognition goes to Milan, who was not only a co-worker but a good friend, and Tim for sweets that greatly helped in writing Chapter 4. 
Thanks to Ove Göttsche, Jaroslav Krystul and Svetlana Polenkova for many pleasant lunches. My good friends from Ukraine also deserve many thanks: Yelena Miroshnichenko, Lyudmila Polyakova and Natalia Yuzva. I would also like to thank Serhiy Chebotaryov for the cover design. Your work is amazing.

Furthermore, I would like to thank the members of my graduation committee for taking their valuable time to read and evaluate my thesis: prof. dr. ir. G.J.M. Krijnen, dr. H.J.W.M. Hoekstra, prof. dr. A.I. Nosich, dr. J.J.G.M. van der Tol, prof. dr. H.P. Urbach, dr. M. Hammer and prof. dr. E.W.C. van Groesen. Your comments and corrections have further improved this thesis.

During our regular NanoNed and Optics meetings I very much appreciated all the discussions with Lasse Kauppinen, Hugo Hoekstra, René de Ridder, Gijs Krijnen, Manfred Hammer, Remco Stoffer, Milan Maksimović and Shahina Mumthaz.

I reserve many thanks to Remco for his understanding, support and patience. It is difficult or even impossible to overestimate his input to my life.

I am very much indebted to my family - mother, father, sister Galya, brother-in-law Kostya, nephew Kirill and small niece Masha. It is their love and devotion which supported me to overcome many obstacles in my life.

\section{Alyona Ivanova}

Enschede, March 2010 


\section{About the author}

Alyona Ivanova was born on the 27th of March 1983 in Kharkiv, Ukraine. She obtained her secondary education at the Lyceum of Physics and Mathematics \#27, Kharkiv, Ukraine in 2000.

From September 2000 until June 2005 she studied Applied Mathematics at V.N. Karazin Kharkiv National University, Kharkiv, Ukraine. The research on her master thesis "Diffraction of an electromagnetic wave by an open cylindrical surface" was performed at the Mathematical Physics and Computational Mathematics group under the supervision of prof. Yuriy V. Gandel.

In January 2006 she started her Ph.D.-research at the Applied Analysis and Mathematical Physics group of the University of Twente, The Netherlands under the supervision of prof. dr. E.W.C. (Brenny) van Groesen and dr. Manfred Hammer. In this research she contributed to the development of a new set of methods for simulation of propagation of light in optical structures. The result of her research is contained in this thesis. 


